\title{
Competition between triplet superconductivity and antiferromagnetism in quasi-one-dimensional electron systems
}

\section{Citation}

Podolsky, Daniel, Ehud Altman, Timofey Rostunov, and Eugene Demler. 2004. “Competition Between Triplet Superconductivity and Antiferromagnetism in Quasi-One-Dimensional Electron Systems." Physical Review B 70 (22) (December 3). doi:10.1103/physrevb.70.224503.

\section{Published Version}

doi:10.1103/physrevb.70.224503

\section{Permanent link}

http://nrs.harvard.edu/urn-3:HUL.InstRepos:27899425

\section{Terms of Use}

This article was downloaded from Harvard University's DASH repository, and is made available under the terms and conditions applicable to Other Posted Material, as set forth at http:// nrs.harvard.edu/urn-3:HUL.InstRepos:dash.current.terms-of-use\#LAA

\section{Share Your Story}

The Harvard community has made this article openly available.

Please share how this access benefits you. Submit a story.

\section{Accessibility}




\title{
Competition between triplet superconductivity and antiferromagnetism in quasi-one-dimensional electron systems
}

\author{
Daniel Podolsky, Ehud Altman, Timofey Rostunov, and Eugene Demler \\ Department of Physics, Harvard University, Cambridge, Massachusetts 02138, USA \\ (Received 4 June 2004; revised manuscript received 6 August 2004; published 3 December 2004)
}

\begin{abstract}
We investigate the competition between antiferromagnetism and triplet superconductivity in quasi-onedimensional electron systems. We show that the two order parameters can be unified using a $\mathrm{SO}(4)$ symmetry and demonstrate the existence of such symmetry in one-dimensional Luttinger liquids of interacting electrons. We argue that approximate $\mathrm{SO}(4)$ symmetry remains valid even when interchain hopping is strong enough to turn the system into a strongly anisotropic Fermi liquid. For unitary triplet superconductors SO(4) symmetry requires a first order transition between antiferromagnetic and superconducting phases. Analysis of thermal fluctuations shows that the transition between the normal and the superconducting phases is weakly first order, and the normal to antiferromagnet phase boundary has a tricritical point, with the transition being first order in the vicinity of the superconducting phase. We propose that this phase diagram explains coexistence regions between the superconducting and the antiferromagnetic phases, and between the antiferromagnetic and the normal phases observed in (TMTSF) ${ }_{2} \mathrm{PF}_{6}$. For nonunitary triplet superconductors the $\mathrm{SO}(4)$ symmetry predicts the existence of a mixed phase of antiferromagnetism and superconductivity. We discuss experimental tests of the $\mathrm{SO}(4)$ symmetry in neutron scattering and tunneling experiments.
\end{abstract}

DOI: 10.1103/PhysRevB.70.224503

PACS number(s): 74.25.Dw, 71.10.Pm, 74.70.Kn

\section{INTRODUCTION}

Quasi-one-dimensional compounds can display a rich variety of phases, including spin-Peierls, charge density wave, spin density wave, and superconducting orders. ${ }^{1-5}$ Due to the large anisotropy in their crystal structure, these materials are often modeled as a collection of weakly coupled Luttinger liquids. The wealth of phases seen in these compounds is then attributed to the intrinsic instability of one-dimensional electron systems towards the formation of quasi long range order. ${ }^{6}$ As temperature is lowered, correlations along individual chains grow, until the coupling between chains stabilize true long range order. In the current paper, we follow this approach to study the interplay between triplet superconductivity (TSC) and antiferromagnetism (AF) in quasi-onedimensional electron systems. The starting point of our discussion is an observation that, for weak umklapp scattering, one-dimensional Luttinger liquids at half-filling have $\mathrm{SO}(4)$ symmetry at the boundary between AF and TSC phases. Near this boundary, the two order parameters can be unified using $\mathrm{SO}(4)$ symmetry, leading to strong constrains on the topology of the phase diagram and on the spectrum of low energy collective excitations.

Our analysis is motivated by quasi-one-dimensional Bechgaard salts (TMTSF) $)_{2} \mathrm{X}$, and their sulphurated counterparts (TMTTF $)_{2} \mathrm{X}$. The most well studied material from this family (TMTSF) ${ }_{2} \mathrm{PF}_{6}$ is an antiferromagnetic insulator at ambient pressure and becomes a superconductor at high pressure. $^{7-11}$ The symmetry of the superconducting order parameter in (TMTSF) ${ }_{2} \mathrm{PF}_{6}$ is not yet fully established, ${ }^{12}$ but there is strong evidence that electron pairing is spin triplet: the superconducting $T_{c}$ is strongly suppressed by disorder; ${ }^{13-17}$ critical magnetic field $H_{c 2}$ in the interchain direction exceeds the paramagnetic limit; ${ }^{18,19}$ the electron spin susceptibility, obtained from the Knight shift measurements, does not decrease below $T_{c}{ }^{20}$ In another material from this family, (TMTSF) ${ }_{2} \mathrm{ClO}_{4}$, superconductivity is stable at ambient pressure and also shows signatures of triplet pairing. ${ }^{21-24}$ Insulator to superconductor transition as a function of pressure has also been found for (TMTSF $)_{2} \mathrm{AsF}_{6}$ (Ref. 25) and $(\mathrm{TMTTF})_{2} \mathrm{PF}_{6} \cdot{ }^{26}$

There are two aspects of the $\mathrm{SO}(4)$ symmetry between antiferromagnetism and triplet superconductivity that we address in this paper.

Classical SO(4) symmetry: We consider the possible emergence of the classical (static) symmetry at a finite temperature critical point. We introduce a Ginzburg-Landau (GL) free energy to describe the interaction between the AF and TSC orders, and we study the effects of thermal fluctuations through a large $N$ expansion and renormalization group (RG) analyses in $d=4-\epsilon$ and $d=2+\epsilon$ dimensions. For a unitary TSC, which we argue to describe Bechgaard salts, we find a first order transition between AF and TSC phases, a first order transition between AF and normal phases ending in a tricritical point, and a weakly first order transition between TSC and normal phases. For a nonunitary TSC we find a mixed phase in which AF and TSC orders are present simultaneously. We argue that the system is close to having an $\mathrm{SO}(4)$ symmetric tetracritical point, but there is a narrow line of direct first order transitions between the normal and the mixed phase. (For a detailed discussion of the distinction between unitary and nonunitary TSC, see Sec. III A.)

Quantum SO(4) symmetry: We introduce a quantum $\mathrm{SO}(4)$ rotor model which encapsulates key features of the competition between AF and TSC orders. We use this model to study collective excitations in the system in various phases. We argue that the $\Theta$-excitation, which gives one of the generators of the $\mathrm{SO}(4)$ algebra, should give rise to a sharp resonance in spin polarized neutron scattering in the TSC phase. We further predict that in the case of a unitary 
TSC the energy of the $\Theta$-resonance should decrease to nearly zero at the phase boundary with the AF phase. Such mode softening is not expected generally near a first order transition and would be a unique signature of the enhanced symmetry at the transition point.

Bechgaard salts belong to a class of strongly correlated electron systems displaying proximity of a superconducting state to some kind of magnetically ordered insulating state. Other examples include the high $T_{c}$ cuprates, ${ }^{27}$ heavy fermion superconductors, ${ }^{28,29}$ and in most cases the superconducting (SC) order parameter is spin singlet ( $s$ or $d$ wave) and the insulating state has antiferromagnetic or spin density wave order. Symmetry principles have been introduced to study the competition of order parameters in some of these systems. In Zhang's $\mathrm{SO}(5)$ theory of high $T_{c}$ superconductivity, ${ }^{30}$ antiferromagnetism and $d$-wave superconductivity are treated as components of a five-dimensional order parameter. In addition to the generators of the usual charge $\mathrm{SO}(2)$ and spin $\mathrm{SO}(3)$ symmetries, new $\pi$-operators are introduced, which rotate superconductivity and antiferromagnetism into each other. A combination of analytical approximations and numerical results can be used to argue an approximate $\mathrm{SO}(5)$ theory of a class of two-dimensional lattice models, such as the Hubbard and the $t$ - $J$ model. ${ }^{31,32}$ The $\mathrm{SO}(5)$ symmetry has also been used to discuss quasi twodimensional organic $\kappa$-BEDT-TTF salts. ${ }^{33}$ The unification approach based on higher symmetries has been generalized to several other types of competing states. $\mathrm{SO}(5)$ and $\mathrm{SO}(8)$ symmetries have been used to classify possible many-body ground states in electronic ladders. ${ }^{34,35} \mathrm{SO}(6)$ symmetry has been introduced to discuss competing striped phases and superconductivity in the cuprates ${ }^{36} \mathrm{SO}(4)$ symmetry has been used to combine $s$-wave superconductivity and charge density wave orders in the negative $U$ Hubbard model, ${ }^{37,38}$ as well as $d$-wave superconductivity and $d$-density wave phases. ${ }^{39,40}$ It was also suggested that the $\mathrm{SO}(5)$ algebra can be used to combine ferromagnetism and triplet superconductivity in quasi-two-dimensional $\mathrm{Sr}_{2} \mathrm{RuO}_{4},{ }^{41}$ although the existence of microscopic models with such symmetry has not been demonstrated.

There are several reasons why Bechgaard salts, and (TMTSF $)_{2} \mathrm{PF}_{6}$ in particular, are promising candidates for experimental observation of the emergence of high symmetry from the competition of two orders. The insulator to superconductor transition in these materials is tuned by pressure, so the entire phase diagram can be explored in a single sample. This compares favorably to the cuprate superconductors, where the AF/SC transition appears as a function of doping and different samples are required to investigate various regimes. Another important advantage of Bechgaard salts is that they may be well described by a microscopic Luttinger liquid Hamiltonian, for which we can demonstrate the existence of $\mathrm{SO}(4)$ symmetry using standard bosonization analysis. This is in contrast to the high $T_{c}$ cuprates, in which approximations need to be made in order to even define generators of the $\mathrm{SO}(5)$ symmetry. ${ }^{30,42,43}$ A related issue is the question of quasiparticles in the AF insulating state and in the $d$-wave superconducting phase. In the former case the quasiparticle spectrum is fully gapped while in the latter case there are nodal quasiparticles. It is not presently known how this difference affects a quantum $\mathrm{SO}(5)$ symmetry for collective bosonic degrees of freedom. An advantage of the $\mathrm{SO}(4)$ symmetry in (TMTSF) ${ }_{2} \mathrm{PF}_{6}$ is that quasiparticles are fully gapped in both the superconducting and the insulating phases.

Recent neutron scattering experiments demonstrated the existence of strong AF fluctuations in a triplet superconductor $\mathrm{Sr}_{2} \mathrm{RuO}_{4} \cdot{ }^{44-46}$ This material is not quasi onedimensional, but it has nested pieces of the Fermi surface (see, e.g., Ref. 44). Thus, we expect that this material may also show some qualitative features of the competition between AF and TSC discussed in this paper.

We note that our approach is phenomenological in nature, since we do not attempt to obtain Luttinger parameters starting from microscopic considerations. Instead, we observe that Bechgaard salts remain strongly anisotropic even close to the AF/TSC phase boundary. Hence, we argue that the Luttinger parameter should be such that individual $1 d$ chains should be in the vicinity of such phase transition. By starting with the Luttinger Hamiltonian, we derive the SO(4) symmetry as its immediate consequence. We note, however, that the Luttinger liquid physics is not a necessary requirement for observing $\mathrm{SO}(4)$ symmetry near the AF/TSC phase boundary. Several groups have argued that near the TSC phase of Bechgaard salts, the interchain tunneling is sufficient to suppress Luttinger liquid behavior in favor of a strongly anisotropic Fermi liquid. ${ }^{47,48}$ We will argue below that an approximate classical $\mathrm{SO}(4)$ symmetry will be present near the AF/ TSC boundary even if the ordered phases arise from a Fermi liquid state, although we still rely on the assumption that interchain hopping of electrons is much smaller than intrachain hopping (this condition is satisfied for Bechgaard salts, see Sec. VIII). Similarly, we expect that the $\Theta$ resonance will be present even in a strongly anisotropic Fermi liquid, whose observation will verify the approximate quantum $\mathrm{SO}(4)$ symmetry. In this paper, for concreteness, we will concentrate on the case where the ordered phases emerge from Luttinger liquid behavior on individual chains.

This paper is organized as follows. In Sec. II we discuss the Luttinger liquid model for interacting electrons in one dimension. For incommensurate band filling, we show that along the transition line between the TSC and the SDW phases, this model has $\mathrm{SO}(3 \times \mathrm{SO}(4)$ symmetry. At halffilling we argue that, for weak umklapp, this symmetry is reduced to $\mathrm{SO}(4)$ symmetry. For quasi-one-dimensional systems such as Bechgaard salts we argue that this SO(4) symmetry provides a unified description of AF and TSC orders. In Sec. III we discuss a general GL free energy for the interplay between magnetism and triplet superconductivity at finite temperatures, and present mean field diagrams for these orders. In Sec. IV we analyze thermal fluctuations using 4 $-\epsilon \mathrm{RG}$ analysis and demonstrate the absence of stable fixed points, which could control multicritical points in the phase diagram. In Sec. V we analyze the case of unitary TSC competing with AF by extending the spin $\mathrm{SO}(3)$ group to a $\mathrm{SO}(N)$ algebra and using large $N$ analysis. In Sec. VI we investigate the interplay of nonunitary TSC and AF using large $N$ approach and $\mathrm{RG}$ analysis for $N>3$ in $4-\epsilon$ and 2 $+\epsilon$ dimensions. We also discuss a physically relevant case of $N=3$. In Sec. VII we introduce an effective $\mathrm{SO}(4)$ quantum 


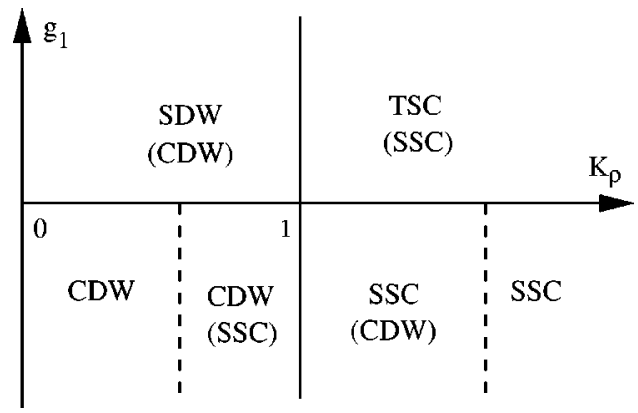

FIG. 1. Phase diagram for a one-dimensional system of interacting spin-1/2 fermions (Ref. 50). Here $K_{\rho}^{2}=\left(2 \pi v_{f}+2 g_{4}+g_{1}\right.$ $\left.-2 g_{2}\right) /\left(2 \pi v_{f}+2 g_{4}-g_{1}+2 g_{2}\right)$. SDW and CDW correspond to spin and charge density wave states, SS and TS to singlet and triplet superconducting phases.

rotor model that condenses the essential features of the competition between the two phases. We use this model to study collective excitations in various phases. In Sec. VIII we discuss $\mathrm{SO}(4)$ symmetry in highly anisotropic Fermi liquids. In Sec. IX we review experimental implications of the $\mathrm{SO}(4)$ symmetry for Bechgaard salts. Finally, in Sec. X we summarize our results.

\section{MICROSCOPIC ORIGIN OF THE SYMMETRY}

\section{A. $\mathrm{SO}(3) \times \mathrm{SO}(4)$ symmetry at incommensurate filling}

Consider a one-dimensional electron gas with the Hamiltonian:

$$
\begin{gathered}
\mathcal{H}=\mathcal{H}_{0}+\mathcal{H}_{1}+\mathcal{H}_{2}+\mathcal{H}_{4} \\
\mathcal{H}_{0}=\sum_{r k s}\left(\epsilon_{r, k s}-\mu\right) a_{r, k s}^{\dagger} a_{r, k s} \\
\mathcal{H}_{1}=\frac{g_{1}}{L} \sum a_{+, k s}^{\dagger} a_{-, p t}^{\dagger} a_{+, p+q t} a_{-, k-q s} \\
\mathcal{H}_{2}=\frac{g_{2}}{L} \sum a_{+, k+q s}^{\dagger} a_{-, p-q t}^{\dagger} a_{-, p t} a_{+, k s} \\
\mathcal{H}_{4}=\frac{g_{4}}{L} \sum a_{+, k+q s}^{\dagger} a_{+, p-q t}^{\dagger} a_{+, p t} a_{+, k s} \\
+\frac{g_{4}}{L} \sum a_{-, k+q s}^{\dagger} a_{-, p-q t}^{\dagger} a_{-, p t} a_{-, k s} .
\end{gathered}
$$

Here, $a_{ \pm, k s}^{\dagger}$ create right/left moving electrons with momenta $\pm k_{f}+k$ and spin $s$, and we assume linearized dispersion of electrons $\epsilon_{r, k s}-\mu=r v_{f} k$. In the Hamiltonian (1) the interaction term $g_{1}$ describes backward scattering and terms $g_{2}$ and $g_{4}$ describe forward scattering. For now, we assume that the system has incommensurate filling, so that umklapp processes are not allowed. The phase diagram for this system obtained from the renormalization group analysis has been discussed extensively before (see, e.g., Refs. 49 and 50) and is shown in Fig. 1.
For the current discussion, we concentrate on the region of the phase diagram near the transition line between the TSC and the SDW phases at $K_{\rho}=1$, i.e.,

$$
g_{1}=2 g_{2} .
$$

We demonstrate that on this line the system has a $\mathrm{SO}(3)$ $\times \mathrm{SO}(4)$ symmetry that unifies order parameters of the two phases.

The total spin operators are defined as

$$
S_{\alpha}=\frac{1}{2} \sum_{r, k s s^{\prime}} a_{r, k s}^{\dagger} \sigma_{s s^{\prime}}^{\alpha}, a_{r, k s^{\prime}},
$$

where $\sigma_{s s^{\prime}}^{\alpha}$ are the usual Pauli matrices. These operators form a spin $\mathrm{SO}(3)$ algebra

$$
\left[S_{\alpha}, S_{\beta}\right]=i \epsilon^{\alpha \beta \gamma} S_{\gamma} .
$$

We can also combine the charge operators for right and left movers $(r= \pm)$ :

$$
Q_{r}=\frac{1}{2} \sum_{k s}\left(a_{r, k s}^{\dagger} a_{r, k s}-\frac{1}{2}\right)
$$

and the operators

$$
\Theta_{r}^{\dagger}=r \sum_{k} a_{r, k \uparrow}^{\dagger} a_{r,-k \downarrow}^{\dagger}
$$

to form two separate isospin $\mathrm{SO}(3)$ algebras

$$
\begin{gathered}
J_{x}^{r}=\frac{1}{2}\left(\Theta_{r}^{\dagger}+\Theta_{r}\right) \\
J_{y}^{r}=\frac{1}{2 i}\left(\Theta_{r}^{\dagger}-\Theta_{r}\right) \\
J_{z}^{r}=Q_{r} \\
{\left[J_{a}^{r}, J_{b}^{r^{\prime}}\right]=i \delta_{r, r^{\prime}} \epsilon^{a b c} J_{c}^{r} .}
\end{gathered}
$$

The total isospin group is therefore $\mathrm{SO}(4)_{\text {isospin }}=\mathrm{SO}(3)_{R}$ $\times \mathrm{SO}(3)_{L}$. Note that, since spin and isospin operators commute, $\left[S_{\alpha}, J_{b}^{r}\right]=0$, they jointly define a closed $\mathrm{SO}(3)_{\text {spin }}$ $\times \mathrm{SO}(4)_{\text {isospin }}$ algebra.

The total spin, $S_{\alpha}$, and the total charge, $Q_{+}+Q_{-}$, always commute with the Hamiltonian (1). In addition, due to the absence of umklapp at incommensurate filling, $Q_{+}$and $Q_{-}$ are conserved separately. As shown in Appendix A using bosonization, when the condition (2) is satisfied, the $\Theta_{r}$ operators also commute with the Hamiltonian. Hence, the system has full $\mathrm{SO}(3) \times \mathrm{SO}(4)$ symmetry at the phase boundary between TSC and SDW phases. We emphasize that the $\mathrm{SO}(3) \times \mathrm{SO}(4)$ symmetry of Luttinger liquids at the SDW/ TSC boundary is generic and does not require fine tuning of the parameters. $\mathrm{SO}(4)_{\text {isospin }}$ invariance has been discussed in quasi one-dimensional systems with highly anisotropic spin interactions. ${ }^{51,52}$ The $\Theta_{r}$ operators in Eq. (6) are reminiscent of the $\eta$ operators introduced by Yang to study the Hubbard model, ${ }^{37,38}$ but we will show in Sec. III B that the two sets of 
operators define different symmetry groups and apply to different systems.

Spin density wave order away from half-filling is described by a complex vector order parameter:

$$
\Phi_{\alpha}=\sum_{k s s^{\prime}} a_{+, k s}^{\dagger} \sigma_{s s^{\prime}}^{\alpha} a_{-, k s^{\prime}} .
$$

For quasi-one-dimensional systems, the band structure restricts the orbital component of the triplet superconducting order to be $\boldsymbol{\Psi}(\mathbf{p}) \propto p_{x}$, where $x$ is the direction parallel to the chains. Thus, the TSC order parameter is also described by a complex vector:

$$
\Psi_{\alpha}^{\dagger}=\frac{1}{i} \sum_{k s s^{\prime}} a_{+, k s}^{\dagger}\left(\sigma^{\alpha} \sigma_{2}\right)_{s s^{\prime}} a_{-,-k s^{\prime}}^{\dagger}
$$

The factor of $-i$ is introduced for convenience, $-i \sigma_{2}$ $\equiv\left(\begin{array}{cc}0 & -1 \\ 1 & 0\end{array}\right)$. The four vector order parameters $\operatorname{Re} \boldsymbol{\Phi}, \operatorname{Im} \boldsymbol{\Phi}$, $\operatorname{Re} \boldsymbol{\Psi}$, and $\operatorname{Im} \Psi$ can be combined into a $4 \times 3$ matrix,

$$
\hat{P}=\left(\begin{array}{llll}
(\operatorname{Re} \boldsymbol{\Psi})_{x} & (\operatorname{Im} \boldsymbol{\Psi})_{x} & (\operatorname{Re} \boldsymbol{\Phi})_{x} & (\operatorname{Im} \boldsymbol{\Phi})_{x} \\
(\operatorname{Re} \boldsymbol{\Psi})_{y} & (\operatorname{Im} \boldsymbol{\Psi})_{y} & (\operatorname{Re} \boldsymbol{\Phi})_{y} & (\operatorname{Im} \boldsymbol{\Phi})_{y} \\
(\operatorname{Re} \boldsymbol{\Psi})_{z} & (\operatorname{Im} \boldsymbol{\Psi})_{z} & (\operatorname{Re} \boldsymbol{\Phi})_{z} & (\operatorname{Im} \boldsymbol{\Phi})_{z}
\end{array}\right) .
$$

Each column of $\hat{P}$ transforms independently as a vector under the action of the spin group,

$$
\left[S_{\alpha}, P_{b \beta}^{-}\right]=i \epsilon^{\alpha \beta \gamma} P_{b \gamma}^{-} .
$$

The action of the isospin group on $\hat{P}$ is easiest to understand in terms of the operators $I_{a}=J_{a}^{+}+J_{a}^{-}$and $\Lambda_{a}=J_{a}^{+}-J_{a}^{-}$. For a fixed row of $\hat{P}$, the action of the isospin generators in the basis $\left(\operatorname{Re} \Psi_{\alpha}, \operatorname{Im} \Psi_{\alpha}, \operatorname{Re} \Phi_{\alpha}, \operatorname{Im} \Phi_{\alpha}\right)$ is represented by

$$
\begin{aligned}
I_{x} & =\left(\begin{array}{cccc}
0 & 0 & 0 & 0 \\
0 & 0 & -i & 0 \\
0 & i & 0 & 0 \\
0 & 0 & 0 & 0
\end{array}\right) \Lambda_{x}=\left(\begin{array}{cccc}
0 & 0 & 0 & -i \\
0 & 0 & 0 & 0 \\
0 & 0 & 0 & 0 \\
i & 0 & 0 & 0
\end{array}\right) \\
I_{y} & =\left(\begin{array}{cccc}
0 & 0 & -i & 0 \\
0 & 0 & 0 & 0 \\
i & 0 & 0 & 0 \\
0 & 0 & 0 & 0
\end{array}\right) \Lambda_{y}=\left(\begin{array}{cccc}
0 & 0 & 0 & 0 \\
0 & 0 & 0 & i \\
0 & 0 & 0 & 0 \\
0 & -i & 0 & 0
\end{array}\right) . \\
I_{z} & =\left(\begin{array}{cccc}
0 & i & 0 & 0 \\
-i & 0 & 0 & 0 \\
0 & 0 & 0 & 0 \\
0 & 0 & 0 & 0
\end{array}\right) \Lambda_{z}=\left(\begin{array}{cccc}
0 & 0 & 0 & 0 \\
0 & 0 & 0 & 0 \\
0 & 0 & 0 & i \\
0 & 0 & -i & 0
\end{array}\right) .
\end{aligned}
$$

Once the fourth component is identified as the "time-like" direction, this is the (Euclidean) Lorentz group, with the $I_{a}$ acting as rotations and the $\Lambda_{a}$ acting as boosts. Hence, the rows of order parameter $P_{\bar{a} \alpha}$ transform in the vector representation of the $\mathrm{SO}(4)_{\text {isospin }}$ group.

\section{B. SO(4) symmetry at half filling}

In Bechgaard salts (TMTSF) ${ }_{2} \mathrm{X}$, three out of every four conduction states are occupied. At quarter filling, umklapp processes involving interactions of four electrons are allowed. Such interactions are weak, and furthermore are irrelevant in the RG sense for $K_{\rho}>1 / 4$ (we remind the readers that we are interested in the regime near the SDW/TSC boundary, where $\left.K_{\rho} \approx 1\right) .{ }^{53}$ On the other hand, due to structural dimerization in Bechgaard salts, ${ }^{54}$ a gap splits the conduction band into a completely filled lower band and a halffilled upper band. Hence, Bechgaard salts are half-filled systems. At half filling, the Hamiltonian (1) must be modified to include two-electron umklapp scattering processes:

$$
\begin{aligned}
\mathcal{H}_{3}= & \frac{g_{3}}{2 L} \sum a_{+, k+q s}^{\dagger} a_{+, p-q t}^{\dagger} a_{-, p t} a_{-, k s} \\
& +\frac{g_{3}}{2 L} \sum a_{-, k+q s}^{\dagger} a_{-, p-q t}^{\dagger} a_{+, p t} a_{+, k s} .
\end{aligned}
$$

Analysis of the phase diagram of Luttinger liquids at halffilling reveals that there is still a direct transition between $\mathrm{AF}$ and TSC orders at $K_{\rho}=1$, although this condition now corresponds ${ }^{2}$ to $g_{1}-2 g_{2}=\left|g_{3}\right|$. The umklapp term allows scattering of two right moving electrons into two left moving ones, and vice versa. Thus, it does not commute with the operator $\Lambda_{z}=Q_{+}-Q_{-}$, which leads to breaking of the $\mathrm{SO}(3)$ $\times \mathrm{SO}(4)$ symmetry. To understand the nature of this symmetry breaking, it is useful to rewrite Eq. (13) in the form

$$
\mathcal{H}_{3}=\frac{g_{3}}{2 L} \sum_{q}[\operatorname{Re} \hat{\boldsymbol{\Phi}}(q) \cdot \operatorname{Re} \hat{\boldsymbol{\Phi}}(-q)-\operatorname{Im} \hat{\boldsymbol{\Phi}}(q) \cdot \operatorname{Im} \hat{\boldsymbol{\Phi}}(-q)],
$$

where $\hat{\boldsymbol{\Phi}}(q)$ is the SDW order parameter at center of mass momentum $2 k_{f}+q$,

$$
\hat{\boldsymbol{\Phi}}(q)=\sum_{k s s^{\prime}} a_{+, k s}^{\dagger} \boldsymbol{\sigma}_{s s^{\prime}} a_{-, k-q s^{\prime}} .
$$

Equation (14) shows explicitly that umklapp tends to pin the phase of the SDW order parameter at either 0 or $\pi$, depending on the sign of $g_{3}$. This is in agreement with the observation that period two antiferromagnetic order can be described by a single real Néel vector.

We will show in Sec. III B that, whereas the GinzburgLandau free energy is no longer $\mathrm{SO}(3)_{\text {spin }} \times \mathrm{SO}(4)_{\text {isospin }}$ symmetric at half-filling, to linear order in $g_{3}$ it maintains a $\mathrm{SO}(4)=\mathrm{SO}(3)_{\text {spin }} \times \mathrm{SO}(3)_{\text {isospin }}$ symmetry. The unbroken part of the isospin group, $\mathrm{SO}(3)_{\text {isospin, }}$, is the diagonal subgroup of $\mathrm{SO}(3)_{R} \times \mathrm{SO}(3)_{L}$, which is generated by the three $I_{a}$ operators, $I_{x}=\frac{1}{2}\left(\Theta^{\dagger}+\Theta\right), I_{y}=1 / 2 i\left(\Theta^{\dagger}-\Theta\right), I_{z}=Q$, where

$$
Q=\frac{1}{2} \sum_{k s}\left(a_{+, k s}^{\dagger} a_{+, k s}+a_{-, k s}^{\dagger} a_{-, k s}-1\right)
$$




$$
\Theta^{\dagger}=\sum_{k}\left(a_{+, k \uparrow}^{\dagger} a_{+,-k \downarrow}^{\dagger}-a_{-, k \uparrow}^{\dagger} a_{-,-k \downarrow}^{\dagger}\right) .
$$

Without loss of generality we consider the case $g_{3}<0$, where the order parameter for antiferromagnetism is given by the real part of $\boldsymbol{\Phi}$,

$$
N_{\alpha}=\frac{1}{2} \sum_{k s s^{\prime}}\left(a_{+, k s}^{\dagger} \sigma_{s s^{\prime}}^{\alpha} a_{-, k s^{\prime}}+a_{-, k s}^{\dagger} \sigma_{s s^{\prime}}^{\alpha} a_{+, k s^{\prime}}\right) .
$$

It is easy to verify that $\{\mathbf{N}, \operatorname{Re} \boldsymbol{\Psi}, \operatorname{Im} \boldsymbol{\Psi}\}$ transform as vectors under both spin and isospin $\mathrm{SO}(3)$ symmetries. We define

$$
\hat{Q}=\left(\begin{array}{ccc}
(\operatorname{Re} \boldsymbol{\Psi})_{x} & (\operatorname{Im} \boldsymbol{\Psi})_{x} & N_{x} \\
(\operatorname{Re} \boldsymbol{\Psi})_{y} & (\operatorname{Im} \boldsymbol{\Psi})_{y} & N_{y} \\
(\operatorname{Re} \boldsymbol{\Psi})_{z} & (\operatorname{Im} \boldsymbol{\Psi})_{z} & N_{z}
\end{array}\right)
$$

$\hat{Q}$ transforms as a vector under both $\mathrm{SO}(3)$ algebras

$$
\begin{gathered}
{\left[I_{a}, Q_{b \beta}\right]=i \epsilon^{a b c} Q_{c \beta}} \\
{\left[S_{\alpha}, Q_{b \beta}\right]=i \epsilon^{\alpha \beta \gamma} Q_{b \gamma},}
\end{gathered}
$$

so it describes an order parameter that transforms as a $(1,1)$ representation of the $\mathrm{SO}(4)$ algebra. Since we are mostly interested in applying our results to Bechgaard salts, we focus mostly on this $\mathrm{SO}(4)$ symmetry in Ref. 55, as well as on the remainder of this paper.

Unlike the $\mathrm{SO}(3) \times \mathrm{SO}(4)$ symmetry discussed in the incommensurate case, the $\mathrm{SO}(4)$ symmetry at half-filling is not a rigorous symmetry of the system. The generators of this group do not commute with the Hamiltonian of the system exactly. However, the main emphasis of our work is to understand the finite temperature phase diagram of (TMTSF) ${ }_{2} \mathrm{PF}_{6}$. This is obtained from the classical GL free energy, which at the AF/TSC phase boundary has $\mathrm{SO}(4)$ symmetry if we retain umklapp processes to linear order in $g_{3}$ (see discussion in Sec. III B). In addition, with regards to quantum properties, $\mathrm{SO}(4)$ symmetry is a good starting point to study the collective modes of the system when $g_{3}$ is small. The latter assumption is well justified for (TMTSF) ${ }_{2} \mathrm{PF}_{6}$, since the observed dimerization in this material is less than $1 \% .{ }^{54}$ For small $g_{3}$, modes found assuming $\mathrm{SO}(4)$ symmetry will have a finite overlap with the actual excitations of the system. In particular, the quantum numbers of the $\Theta$ mode discussed in Sec. VII, including charge two and center of mass momentum $2 k_{f}$, are not affected by umklapp. These properties determine which experimental probes couple to $\Theta$. We must keep in mind, however, that the explicit breaking of $\mathrm{SO}(4)$ due to higher order corrections in $g_{3}$, and also due to interchain coupling, may give a small energy gap and finite broadening to $\Theta$, even at the AF/TSC phase boundary. We also point out that, from the point of view of $\Theta$ excitations, the difference between $\mathrm{SO}(3) \times \mathrm{SO}(4)$ and $\mathrm{SO}(4)$ symmetries corresponds to the question whether $+2 k_{f}$ and $-2 k_{f}$ excitations are the same [SO(4) symmetry at half filling] or different $[\mathrm{SO}(3) \times \mathrm{SO}(4)$ symmetry away from half filling].

Thus far in the analysis we have ignored spin-orbit effects. Microwave absorption experiments in (TMTSF) ${ }_{2} \mathrm{AsF}_{6}$ measured $^{56}$ the anisotropy in the exchange couplings to be

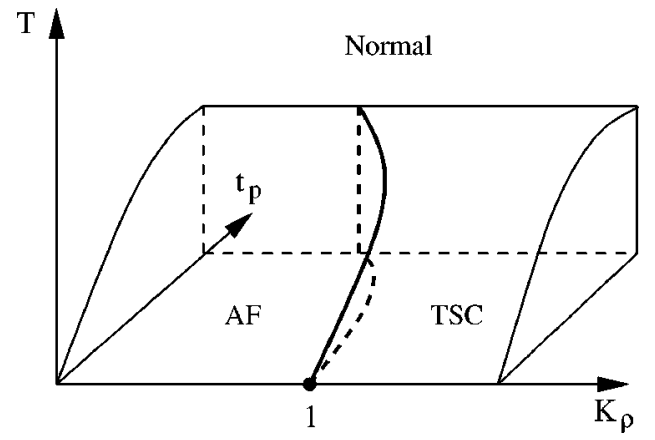

FIG. 2. Proposed phase diagram for weak interchain coupling $t_{b}$. When interchain coupling is present, $t_{b} \neq 0$, long range order at finite temperatures becomes possible. In the unitary case, the second order quantum critical point of a Luttinger liquid becomes a first order transition between AF and unitary TSC. As $t_{b}$ grows, the AF phase shrinks due to reduced nesting of the Fermi surface. Throughout we assume positive backscattering $g_{1}>0$.

$10^{-6}$. This ultimately determines the preferred axes for the Néel vector $\mathbf{N}$ (along the $b$ axis of the crystal ${ }^{57}$ ) and the spin component of the TSC order $\boldsymbol{\Psi}$ (along either the $a$ or $c$ axis $^{18}$ ). However, we do not expect such tiny anisotropy to play a significant role in determining the competition between AF and TSC phases. We also point out that NMR experiments in (TMTSF) ${ }_{2} \mathrm{PF}_{6}$ find a divergence of $T_{1}^{-1}$ at the Néel temperature that is well-described by the $\mathrm{O}(3)$ isotropic Heisenberg model. ${ }^{58}$ Thus, even for critical fluctuations of the AF order parameter, spin anisotropy coming from spinorbit coupling is unobservably small.

Before concluding this section we point out that the isospin algebra defined by Eqs. (5)-(7) can also be used to relate charge density wave order and singlet superconductivity in quasi-one-dimensional electron systems. ${ }^{59}$ This is relevant for the lower half of Fig. 1.

\section{GINZBURG-LANDAU FREE ENERGY}

The main goal of this section is to investigate consequences of the $\mathrm{SO}(4)$ symmetry for the true finite temperature phase transitions, when we need to consider threedimensional fluctuations of the order parameter. One may be concerned that by introducing interchain couplings, we will immediately destroy the $\mathrm{SO}(4)$ symmetry. As we will show in Sec. VIII, even in the case where the interchain coupling $t_{b}$ is large enough to make the system into a highly anisotropic Fermi liquid, approximate $\mathrm{SO}(4)$ symmetry prevails in the Ginzburg-Landau (GL) free energy [see, e.g., Eq. (29)]. This feature of the AF/TSC GL free energy implies that our analysis of the phase diagram, based on classical SO(4) symmetry, is valid even when the normal state is described by a highly anisotropic Fermi liquid rather than a collection of weakly coupled Luttinger liquids. A review of the normal state properties of organic superconductors at low magnetic fields is given in Ref. 47, and low temperature transport properties have been reported recently in Ref. 48 .

We illustrate the effects of interchain coupling in Fig. 2. As the temperature is reduced on either side of $K_{\rho}=1$, the 
correlation length along a chain in the appropriate correlation function grows due to intrachain interactions. At some finite temperature, before this length diverges, coupling between the chains becomes relevant and a true three-dimensional transition can take place. At the crossover between one and three dimensions, the description of the system in terms of a Luttinger liquid is supplanted by a GL free energy describing the interactions of the order parameters in three spatial dimensions. In this picture, for small enough $t_{b}$ the presence of a phase boundary between AF and TSC implies that the intrachain Hamiltonian is close to the $\mathrm{SO}(4)$ symmetric point $K_{\rho}=1$.

An important assumption of our analysis is that pressure varies the value of $K_{\rho}$ and tunes the transition across the AF/TSC phase boundary. We note that measurements of $K_{\rho}$ based on optical conductivity measurements have been carried out at ambient pressure, i.e., deep inside the SDW phase in (TMTSF) ${ }_{2} \mathrm{PF}_{6}$. For instance, in Refs. 60 and 61, the value $K_{\rho}=0.23$ is obtained. Reference 61 points out that this value of $K_{\rho}$ assumes that the dominant umklapp contribution is due to commensurability at quarter filling. They acknowledge that if umklapp is dominated by commensurability at halffilling, these measurements then imply $K_{\rho}=0.925$. They also point out that a half-filled model has the drawback of predicting a gap energy that is too small. However, it is equally difficult to justify the assumption that quarter filling commensurability is dominant: for $K_{\rho}=0.23$, the rate of divergence in the RG of commensurability at one quarter is exponentially smaller than the corresponding rate for commensurability at half-filling. Thus, we feel that the question of the value of $K_{\rho}$ is not yet settled.

\section{A. Incommensurate filling}

At incommensurate filling, a translation by one lattice constant multiplies the SDW order parameter by a complex phase factor:

$$
\boldsymbol{\Phi} \rightarrow e^{2 i \mathbf{k}_{\mathbf{f}} \cdot \mathbf{a}} \mathbf{\Phi}=e^{2 \pi i v} \boldsymbol{\Phi},
$$

where $\nu$ is the filling fraction of the conduction band. For a completely incommensurate case, when $\nu$ is an irrational number, the GL free energy must be $\mathrm{SO}(2)$ symmetric with respect to the phase of $\boldsymbol{\Phi} .{ }^{62,63}$ In the absence of pinning terms, the most general GL free energy with $\mathrm{SO}(3)_{\text {spin }}$ $\times \mathrm{SO}(2)_{\text {charge }} \times \mathrm{SO}(2)_{\text {translation }}$ is

$$
\begin{aligned}
F= & \frac{1}{2}|\nabla \boldsymbol{\Psi}|^{2}+\frac{1}{2}|\nabla \boldsymbol{\Phi}|^{2}+\frac{r_{1}}{2}|\boldsymbol{\Psi}|^{2}+\frac{r_{2}}{2}|\boldsymbol{\Phi}|^{2}+u_{1}\left(|\boldsymbol{\Psi}|^{2}\right)^{2} \\
& +u_{2}\left(|\boldsymbol{\Phi}|^{2}\right)^{2}+u_{3}\left|\boldsymbol{\Psi}^{2}\right|^{2}+u_{4}\left|\boldsymbol{\Phi}^{2}\right|^{2}+2 v_{1}|\boldsymbol{\Psi}|^{2}|\boldsymbol{\Phi}|^{2} \\
& +2 v_{2}|\boldsymbol{\Phi} \cdot \boldsymbol{\Psi}|^{2}+2 v_{3}|\boldsymbol{\Phi} * \cdot \boldsymbol{\Psi}|^{2} .
\end{aligned}
$$

Near the phase boundary between SDW and TSC phases, for quasi-one-dimensional systems the form of the free energy is strongly constrained by the $\mathrm{SO}(3)_{\text {spin }} \times \mathrm{SO}(4)_{\text {isospin }}$ symmetry. We expect the properties of the system to be well described by the free energy,

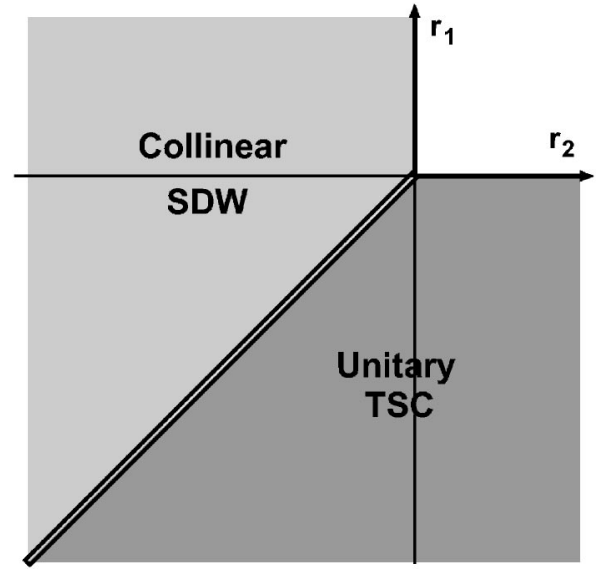

FIG. 3. Mean-field phase diagram of Eqs. (22) and (29) for $\tilde{u}_{2}<0$. At half filling, SDW order reduces to AF order.

$$
\begin{aligned}
F= & \frac{1}{2} \sum_{\bar{a} \alpha} \nabla P_{\bar{a} \alpha} \nabla P_{\bar{a} \alpha}+\frac{\bar{r}}{2} \sum_{\bar{a} \alpha} P_{\bar{a} \alpha} P_{\bar{a} \alpha}+\frac{\delta r}{2} \sum_{\alpha}\left(P_{1 \alpha}^{2}+P_{2 \alpha}^{2}\right. \\
& \left.-P_{3 \alpha}^{2}-P_{4 \alpha}^{2}\right)+\widetilde{u}_{1} \sum_{\bar{a} \alpha \bar{b} \beta} P_{\bar{a} \alpha} P_{\bar{a} \alpha} P_{\bar{b} \beta} P_{\bar{b} \beta}^{-} \\
& +\widetilde{u}_{2} \sum_{\bar{a} \alpha \bar{b} \beta} P_{\bar{a} \alpha} P_{\bar{a} \beta} P_{\bar{b} \alpha}^{-} P_{\bar{b} \beta}^{-} .
\end{aligned}
$$

This is the most general free energy with $\mathrm{SO}(3)_{\text {spin }}$ $\times \mathrm{SO}(4)_{\text {isospin }}$ symmetric quartic coefficients, where we have used the order parameter defined in Eq. (10) to display this invariance explicitly. We follow the common assumption that changing the external control parameters of the system only affects the quadratic coefficients. Thus, these are allowed to break the symmetry and tune the phase transition. For $\delta r$ $\neq 0$, the symmetry is broken down to $\mathrm{SO}(3)_{\text {spin }}$ $\times \mathrm{SO}(2)_{\text {charge }} \times \mathrm{SO}(2)_{\text {translation }}$.

There is an explicit duality between antiferromagnetism and triplet superconductivity under reversal of the sign of $\delta r$ in Eq. (22). The mean field phase diagram of Eq. (22) depends crucially on the sign of $\widetilde{u}_{2}$. For negative $\widetilde{u}_{2}$, there is a tendency for all vector order parameters to point along a common axis. The order parameters in this case can be described by a single real vector times a complex phase, $\boldsymbol{\Psi}$ $=e^{i \varphi} \mathbf{n}$ and $\boldsymbol{\Phi}=e^{i \theta} \mathbf{n}$. This is referred to in the ${ }^{3} \mathrm{He}$ literature as unitary triplet superconductivity, ${ }^{64}$ and in magnetism as collinear spin density order. ${ }^{62}$ On the other hand, for positive $\widetilde{u}_{2}$, all vector order parameters tend to be mutually orthogonal. The real and imaginary parts of the order parameters can no longer be set to be parallel, $\operatorname{Re} \boldsymbol{\Psi} \times \operatorname{Im} \boldsymbol{\Psi} \neq 0$ and $\operatorname{Re} \boldsymbol{\Phi}$ $\times \operatorname{Im} \boldsymbol{\Phi} \neq 0$. This is the nonunitary/noncollinear case. The mean-field phase diagrams for Eq. (22) for $\widetilde{u}_{2}$ negative and positive are shown in Figs. 3 and 4.

In principle, the parameters of the GL free energy Eq. (21) can be obtained from a microscopic Hamiltonian. In Appendix B we consider a quasi-one-dimensional electron systems with weak interactions and obtain a free energy as in Eq. (22) with

$$
\tilde{u}_{1}=\frac{21 \zeta(3)}{16 \pi^{2} v_{f} T^{2}}
$$




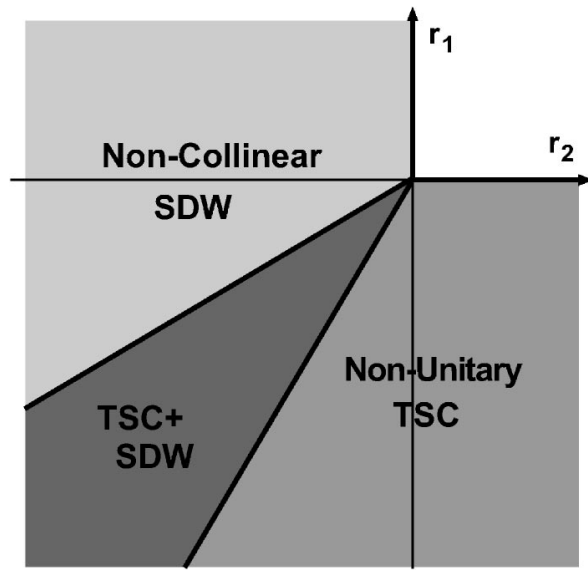

FIG. 4. Mean-field phase diagram of Eqs. (22) and (29) for $\widetilde{u}_{2}>0$. At half filling, SDW order reduces to AF order.

$$
\tilde{u}_{2}=-\frac{7 \zeta(3)}{8 \pi^{2} v_{f} T^{2}} .
$$

The quadratic coefficients depend on coupling constants in the TSC and SDW channels, and on temperature. They are typically parameterized in terms of the pressure-dependent mean-field transition temperature

$$
\begin{gathered}
r_{1}(T, P)=\alpha_{\mathrm{TSC}}\left[T-T_{C}(P)\right] \\
r_{2}(T, P)=\alpha_{\mathrm{SDW}}\left[T-T_{N}(P)\right] .
\end{gathered}
$$

The analysis of Appendix B shows that weakly interacting Fermi liquids favor unitary TSC and collinear SDW order, $\tilde{u}_{2}<0$.

\section{B. Half-filling}

As is well known, the physics of period two antiferromagnetic order is captured by a single real Néel vector. It is interesting to study how this comes about from the point of view of the microscopic Luttinger Hamiltonian. As pointed out in Sec. II, the Hamiltonian at half-filling includes a new contribution due to umklapp scattering, Eq. (14). This gives a correction to the GL free energy, which to linear order in $g_{3}$ can be written as

$$
\Delta F=h\left[(\operatorname{Re} \boldsymbol{\Phi})^{2}-(\operatorname{Im} \boldsymbol{\Phi})^{2}\right],
$$

where $h=g_{3} / 2 L$. The new term pins the SDW, and breaks the $\mathrm{SO}(4)_{\text {isospin }}=\mathrm{SO}(3)_{R} \times \mathrm{SO}(3)_{L}$ symmetry down to its diagonal subgroup $\mathrm{SO}(3)_{\text {isospin. }}$ In agreement with the FeynmanHellman theorem, the quartic coefficients derived in Appendix B are not modified to linear order in $g_{3}$. Therefore, in the linear order in $g_{3}$, the free energy has $\mathrm{SO}(4)=\mathrm{SO}(3)_{\text {spin }}$ $\times \mathrm{SO}(3)_{\text {isospin }}$ symmetry. In principle, the higher order contributions of the umklapp $g_{3}$ term can break the original $\mathrm{SO}(4)_{\text {isospin }}$ symmetry all the way down to $\mathrm{SO}(2)_{\text {charge }}$, generated by the total charge $Q$.

The bare value of $g_{3}$ in (TMTSF) ${ }_{2} \mathrm{PF}_{6}$ is small, since it is proportional to dimerization, which in this compound is very weak. ${ }^{54}$ Assuming that Coulomb interactions are of the order of the bandwidth, this leads to a bare value of $g_{3}$ of about 0.01 . Furthermore, it is not the bare value of $g_{3}$ that enters the GL free energy, but its effective (renormalized) value at the $1 d-3 d$ crossover scale. At high temperatures onedimensional physics is observed. As temperature is reduced, everywhere on the TSC side of the phase diagram, as well as on the AF/TSC phase boundary, $g_{3}$ flows to zero. This allows us to approach the critical region from the TSC side, along which the GL free energy is $\mathrm{SO}(3)_{\text {iso }}$ symmetric. Even on the AF side of the phase diagram, where $g_{3}$ is relevant, the flow of $g_{3}$ passes near zero before diverging. Therefore, near the AF/TSC phase boundary, the flow spends a lot of time near zero, and the eventual upturn of $g_{3}$ may not be reached for realistic systems, in which the $3 d$ coupling may cut off the $1 d \mathrm{RG}$ flow at low temperatures. Hence, it is reasonable to take small $g_{3}$ everywhere near the AF/TSC phase boundary, and to consider a model with $\mathrm{SO}(3)_{\text {iso }}$ symmetry.

In what follows, we will assume that the umklapp term favors the real part of the SDW, which becomes the Néel order parameter $\mathbf{N}$ :

$$
\mathbf{N}=\operatorname{Re} \boldsymbol{\Phi} .
$$

From now on we assume that $\operatorname{Im} \boldsymbol{\Phi}$ is sufficiently well gapped, so that it does not need to be included in the analysis of the competition between AF and TSC. This is justified since the pinning term Eq. (25) is relevant in the $3 d$ theory, and thus any fixed point of the theory will be characterized by strong pinning.

It is useful to consider the relation between our $\mathrm{SO}(4)$ symmetry and the $\mathrm{SO}(4)$ symmetry introduced by Yang for the Hubbard model. ${ }^{65}$ The symmetry generators of Yang's $\mathrm{SO}(4)$ is the $\eta$ operator:

$$
\eta^{\dagger}=\sum_{k} c_{k+\pi \uparrow}^{\dagger} c_{-k \downarrow}^{\dagger}
$$

$k$ summation goes over the entire Brillouin zone. This operator should be compared to our $\Theta$ operators defined in Eq. (6) for incommensurate filling, and Eq. (16) for half-filling. Away from half filling, the difference between the two operators is obvious. $\Theta$ has momentum equal to the nesting wave vector $2 k_{f}$, in contrast to $\eta$, which always has center of mass momentum $\pi$. This allows us to have $\mathrm{SO}(4)$ symmetry for any electron density, in contrast to Yang's $\mathrm{SO}(4)$, which only applies at half-filling. At half-filling, however, $2 k_{f}=\pi$, and the only difference is the relative sign between the left and right moving contributions. This difference is substantial. The Néel order parameter transforms as a singlet under the action of $\eta$ :

$$
[\eta, \mathbf{N}]=0 \text {. }
$$

This should be contrasted with $\Theta$, which rotates $\mathbf{N}$ into the TSC order parameter $\boldsymbol{\Psi}$, see Eq. (19). The two SO(4) symmetries thus differ in the order parameters which they unify, and in the microscopic models for which they apply. Yang's $\mathrm{SO}(4)$ applies to the negative $U$ Hubbard model, for which singlet SC and CDW are degenerate lowest energy states at half-filling. Our SO(4) unifies AF and TSC orders, which are not degenerate for the Hubbard model. As we discussed earlier, we expect these to be nearly degenerate phases for half- 
filled systems with small umklapp [e.g., quarter filled systems with small dimerization, such as $\left(\mathrm{TMTSF}_{2}\right) \mathrm{PF}_{6}$ and with $K_{\rho}$ close to one].

In analogy with the incommensurate case, at half-filling we expect that quasi-one-dimensional systems near the AF/ TSC phase boundary have a Ginzburg-Landau free energy with $\mathrm{SO}(4)$-symmetric quartic coefficients. The symmetry can be made explicit in terms of the matrix order parameter $\hat{Q}$ :

$$
\begin{aligned}
F= & \frac{1}{2} \sum_{a \alpha} \nabla Q_{a \alpha} \nabla Q_{a \alpha}+\frac{\hat{r}}{2} \sum_{a \alpha} Q_{a \alpha} Q_{a \alpha}+\delta r \sum_{\alpha}\left(Q_{3 \alpha}^{2}-Q_{1 \alpha}^{2}\right. \\
& \left.-Q_{2 \alpha}^{2}\right)+\widetilde{u}_{1} \sum_{a \alpha b \beta} Q_{a \alpha} Q_{a \alpha} Q_{b \beta} Q_{b \beta}+\widetilde{u}_{2} \sum_{a \alpha b \beta} Q_{a \alpha} Q_{a \beta} Q_{b \alpha} Q_{b \beta} \\
= & \frac{1}{2}|\nabla \boldsymbol{\Psi}|^{2}+\frac{1}{2}(\nabla \mathbf{N})^{2}+\frac{r_{1}}{2}|\boldsymbol{\Psi}|^{2}+\frac{r_{2}}{2} \mathbf{N}^{2}+\left(\widetilde{u}_{1}+\frac{\widetilde{u}_{2}}{2}\right)\left(|\boldsymbol{\Psi}|^{2}\right)^{2} \\
& +\frac{\widetilde{u}_{2}}{2}\left|\boldsymbol{\Psi}^{2}\right|^{2}+2 \widetilde{u}_{1}|\boldsymbol{\Psi}|^{2} \mathbf{N}^{2}+2 \widetilde{u}_{2}|\boldsymbol{\Psi} \cdot \mathbf{N}|^{2}+\left(\widetilde{u}_{1}+\widetilde{u}_{2}\right)\left(\mathbf{N}^{2}\right)^{2} .
\end{aligned}
$$

Changing temperature and some other parameter of the system [e.g., pressure in $\left(\mathrm{TMTSF}_{2}\right) \mathrm{PF}_{6}$ ] allows to control $r_{1}$ and $r_{2}$. The $\mathrm{SO}(4)$ symmetry is recovered on the line $r_{1}=r_{2}$.

Equation (29) is a special case of the most general free energy with the $\mathrm{SO}(3) \times \mathrm{SO}(2)$ symmetry of spin and charge rotations, ${ }^{66}$

$$
\begin{aligned}
\widetilde{F}= & \frac{1}{2}|\nabla \boldsymbol{\Psi}|^{2}+\frac{1}{2}(\nabla \mathbf{N})^{2}+\frac{r_{1}}{2}|\boldsymbol{\Psi}|^{2}+\frac{r_{2}}{2} \mathbf{N}^{2}+u_{1}\left(|\boldsymbol{\Psi}|^{2}\right)^{2} \\
& +u_{2}\left(\mathbf{N}^{2}\right)^{2}+u_{3}\left|\Psi^{2}\right|^{2}+2 v_{1}|\Psi|^{2} \mathbf{N}^{2}+2 v_{2}|\mathbf{N} \cdot \boldsymbol{\Psi}|^{2}
\end{aligned}
$$

Translational symmetry rules out $\mathbf{N} \cdot \boldsymbol{\Psi} * \times \boldsymbol{\Psi}$ because this term has a nonzero wave vector. Similarly, $|\mathbf{N} \times \boldsymbol{\Psi}|^{2}$ can be reduced to terms already present in Eq. (30). When the conditions

$$
\begin{gathered}
r_{1}=r_{2} \\
u_{2}-u_{3}=u_{1} \\
u_{2}-2 u_{3}=v_{1} \\
v_{2}=2 u_{3}
\end{gathered}
$$

are satisfied, we recover $\mathrm{SO}(4)$ symmetry. In addition, if we supplement the conditions (31) by

$$
v_{2}=0
$$

there is an even higher $\mathrm{SO}(9)$ symmetry, which allows rotations between any components of vectors $\mathbf{N}, \operatorname{Re} \boldsymbol{\Psi}$, and $\operatorname{Im} \boldsymbol{\Psi}$ :

$$
\bar{F}=\frac{1}{2}|\nabla \boldsymbol{\Psi}|^{2}+\frac{1}{2}(\nabla \mathbf{N})^{2}+\frac{r}{2}\left(|\boldsymbol{\Psi}|^{2}+\mathbf{N}^{2}\right)+\bar{u}\left(|\boldsymbol{\Psi}|^{2}+\mathbf{N}^{2}\right)^{2} .
$$

At half-filling, there is no distinction between collinear and noncollinear magnetism. However, the sign of $\widetilde{u}_{2}$ still determines the nature of the triplet superconductivity, as well as the topology of the mean-field phase diagrams. These are similar to those displayed for incommensurate filling, Figs. 3 and 4, the only difference being that SDW order is reduced to AF order. The mean-field phase diagrams for Eq. (29) for $\widetilde{u}_{2}$ negative and positive are shown in Fig. 3. The analysis of Appendix B can be easily modified to half-filling, and yields a $\mathrm{SO}(4)$ symmetric free energy of the form Eq. (29) with coefficients still given by Eq. (23). Thus, weekly interacting Fermi liquids favor the case $\widetilde{u}_{2}<0$. Strong interactions, however, can modify the quartic coefficients Eq. (29), including a possible change of sign of $\widetilde{u}_{2}$. In the subsequent discussion we consider both possibilities. It is useful to note that all experimentally known cases of triplet pairing between fermions, such as ${ }^{3} \mathrm{He},{ }^{64,67}$ and $\mathrm{Sr}_{2} \mathrm{RuO}_{4}$, correspond to the unitary case. Hence, negative $\widetilde{u}_{2}$ appears more likely.

\section{THERMAL FLUCTUATIONS}

We now consider the free energy (30) and address how fluctuations affect the mean-field phase diagram shown in Figs. 3 and 4. For instance, when the quartic coefficients do not lie exactly on the SO(4) symmetric manifold, we will study whether such symmetry appears as we go to longer length scales and integrate out short wave length fluctuations. The possibility of enhanced static symmetry at the critical point has been discussed previously for several solid state systems. For easy axis AF in a magnetic field, $\mathrm{SO}(3)$ symmetry was suggested to appear at the spin flop critical point. ${ }^{68,69}$ For systems with competing singlet superconducting and antiferromagnetic orders Zhang suggested a static $\mathrm{SO}(5)$ symmetry as the bicritical point. ${ }^{30,70}$ This $\mathrm{SO}(5)$ symmetry has also been used to study the quasi-two-dimensional $\kappa$-BEDT-TTF salts. ${ }^{33}$ Yang and Zhang introduced a $\mathrm{SO}(4)$ symmetry for the Hubbard model at half-filling which unifies singlet superconductivity with charge density wave order. $^{37,38}$

To understand the role of fluctuations in models (30) and (29) we use $4-\epsilon$ renormalization group (RG) analysis. For subsequent discussion it is useful to extend the spin $\mathrm{SO}(3)$ symmetry of the Eq. (30) to a more general $\mathrm{SO}(\mathrm{N})$ symmetry. This is achieved by considering vectors $\mathbf{N}$ and $\boldsymbol{\Psi}$ as $\mathrm{N}$-component vectors. The RG equations can be derived using the standard approach ${ }^{71}$

$$
\begin{gathered}
\frac{d r_{1}}{d l}=2 r_{1}+\frac{8 K_{d}}{1+r_{1}}\left[(N+1) u_{1}+3 u_{3}\right]+\frac{4 K_{d}}{1+r_{2}}\left[N v_{1}+2 v_{2}\right] \\
\frac{d r_{2}}{d l}=2 r_{2}+\frac{8 K_{d}}{1+r_{1}}\left[N v_{1}+2 v_{2}\right]+\frac{4 K_{d}}{1+r_{2}}(N+2) u_{2} \\
\frac{d u_{1}}{d l}=\epsilon u_{1}-K_{d}\left[(8 N+32) u_{1}^{2}+32 u_{1} u_{3}+32 u_{3}^{2}+4 N v_{1}^{2}+8 v_{1} v_{2}\right. \\
\left.+2 v_{2}^{2}\right] \\
\frac{d u_{2}}{d l}=\epsilon u_{2}-K_{d}\left[4(N+8) u_{2}^{2}+8 N v_{1}^{2}+16 v_{1} v_{2}+8 v_{2}^{2}\right]
\end{gathered}
$$




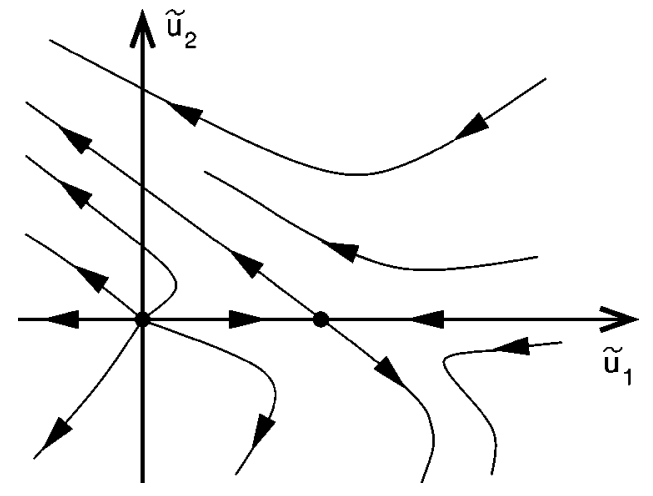

FIG. 5. Renormalization group flow of the $\mathrm{SO}(4)$ symmetric theory Eq. (29) in $d=4-\varepsilon$ dimensions. The sign of $\widetilde{u}_{2}$ does not change under the flow, and there are no stable fixed points. Instead, there are two types of runaway flow, corresponding to unitary $\left(\tilde{u}_{2}<0\right)$ and nonunitary $\left(\tilde{u}_{2}>0\right)$ TSC. The two are separated by a line of $\operatorname{SO}(9)$ symmetric theories $\left(\widetilde{u}_{2}=0\right)$.

$$
\begin{gathered}
\frac{d u_{3}}{d l}=\epsilon u_{3}-K_{d}\left[8 N u_{3}^{2}+48 u_{1} u_{3}+2 v_{2}^{2}\right] \\
\frac{d v_{1}}{d l}=\epsilon v_{1}-K_{d}\left[(8 N+8) u_{1} v_{1}+(4 N+8) u_{2} v_{1}+16 u_{3} v_{1}+16 v_{1}^{2}\right. \\
\left.+8 u_{1} v_{2}+4 v_{2} u_{2}+4 v_{2}^{2}\right] \\
\frac{d v_{2}}{d l}=\epsilon v_{2}-K_{d}\left[8 u_{1} v_{2}+8 u_{2} v_{2}+16 u_{3} v_{2}+32 v_{1} v_{2}\right. \\
\left.+(4 N+8) v_{2}^{2}\right]
\end{gathered}
$$

Here $d l=d \Lambda / \Lambda$, where $\Lambda$ is a momentum cutoff, and $K_{d}$ $=\ldots$ is a surface of a unit sphere in $d=4-\epsilon$ dimension.

For the physically relevant $N=3$, Eq. (34) has only two fixed points. One is a trivial Gaussian fixed point

$$
r_{\{1,2\}}=u_{\{1,2,3\}}=v_{\{1,2\}}=0
$$

and the other is a $\mathrm{SO}(9)$ Heisenberg point

$$
\begin{gathered}
r_{\{1,2\}}=-\frac{(3 N+2) \epsilon}{6 N+16} \\
u_{1}=u_{2}=v_{1}=\frac{\epsilon}{(6 N+16) K_{d}} \\
u_{3}=v_{2}=0 .
\end{gathered}
$$

The Gaussian fixed point is completely unstable. The $\mathrm{SO}(9)$ Heisenberg point has five unstable directions [for general $N$, the Heisenberg point has $\mathrm{SO}(3 N)$ symmetry, but it remains unstable in five directions for all $N>1$ ]. The critical point should have only two unstable directions: $r_{(1,2)}$ should flow away from the critical point, but all the interaction coefficient should flow toward the fixed point. So, neither the Gaussian nor the $\mathrm{SO}(9)$ Heisenberg fixed points are good candidates for the critical point. In Fig. 5 we show RG flows in the $\mathrm{SO}(4)$ symmetric plane. We find two types of runaway flows. When we start with $\widetilde{u}_{2}$ positive, it continues increasing. For $\widetilde{u}_{2}$ negative, the RG flow makes it even more negative. In both cases $\widetilde{u}_{1}$ flows to negative values.

In many cases absence of a fixed point in the RG flows implies that we do not have a multicritical point in the phase diagram, but instead fluctuations induce a first order phase transition. Below we discuss consequences of the runaway flows in Eq. (34). We point out that two types of the runaway flows in the $\mathrm{SO}(4)$ symmetric manifold shown in Fig. 5 correspond to unitary $\left(\tilde{u}_{2}<0\right)$ and nonunitary $\left(\tilde{u}_{2}>0\right)$ TSC. These two cases are considered separately.

\section{FINITE TEMPERATURE ANALYSIS: UNITARY CASE}

We consider model Eq. (29) with $N$-component vectors and with negative $\widetilde{u}_{2}$. In the $4-\epsilon$ expansion there are runaway flows even for large $N$. Thus, we do not find a fixed point that could give a critical point. To understand the phase diagram in this case we employ large $N$ calculations in $d$ $=3$. In the large $N$ expansion, all bubble diagrams are summed self-consistently. ${ }^{72,73}$ The large $N$ approach for unitary triplet superconductors without coupling to magnetic order has been discussed previously in Ref. 74 in the context of ${ }^{3} \mathrm{He}$.

Let us start by analyzing the superconducting phase. In the mean-field approximation the order parameter factorizes as $\boldsymbol{\Psi}=e^{i \theta} \mathbf{n}$. Hence, we take the average value of the order parameter in the ordered phase to be $\boldsymbol{\Psi}_{0}=(0, \ldots, 0, \sigma)$ and separate the longitudinal and transverse components of the fluctuating part $\delta \mathbf{\Psi}=\left(\mathbf{A}_{T}+i \mathbf{B}_{T}, A_{L}+i B_{L}\right)$. For the Néel order parameter we also separate $\mathbf{N}=\left(\mathbf{N}_{T}, N_{L}\right)$. Effective masses for $\mathbf{A}_{T}, \mathbf{B}_{T}$, and $\mathbf{N}_{T}$ are given by

$$
\begin{aligned}
r_{A}= & r_{1}+4\left(\widetilde{u}_{1}+\widetilde{u}_{2}\right) \sigma^{2}+4\left(\widetilde{u}_{1}+\widetilde{u}_{2}\right) N \int_{0}^{\Lambda} \frac{d^{3} k}{(2 \pi)^{3}} \frac{1}{k^{2}+r_{A}} \\
+ & 4 \widetilde{u}_{1} N \int_{0}^{\Lambda} \frac{d^{3} k}{(2 \pi)^{3}}\left(\frac{1}{k^{2}+r_{B}}+\frac{1}{k^{2}+r_{N}}\right) \\
r_{B}= & r_{1}+4 \widetilde{u}_{1} \sigma^{2}+4\left(\widetilde{u}_{1}+\widetilde{u}_{2}\right) N \int_{0}^{\Lambda} \frac{d^{3} k}{(2 \pi)^{3}} \frac{1}{k^{2}+r_{B}} \\
& +4 \widetilde{u}_{1} N \int_{0}^{\Lambda} \frac{d^{3} k}{(2 \pi)^{3}}\left(\frac{1}{k^{2}+r_{A}}+\frac{1}{k^{2}+r_{N}}\right), \\
r_{N}= & r_{2}+4 \widetilde{u}_{1} \sigma^{2}+4\left(\widetilde{u}_{1}+\widetilde{u}_{2}\right) N \int_{0}^{\Lambda} \frac{d^{3} k}{(2 \pi)^{3}} \frac{1}{k^{2}+r_{N}} \\
& +4 \widetilde{u}_{1} N \int_{0}^{\Lambda} \frac{d^{3} k}{(2 \pi)^{3}}\left(\frac{1}{k^{2}+r_{A}}+\frac{1}{k^{2}+r_{B}}\right)
\end{aligned}
$$

where $\Lambda$ is the ultraviolet (short distance) cutoff of the free 


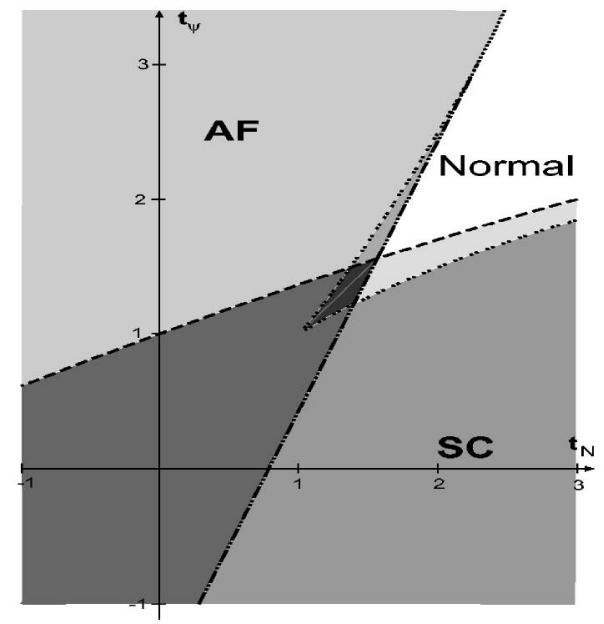

(a)

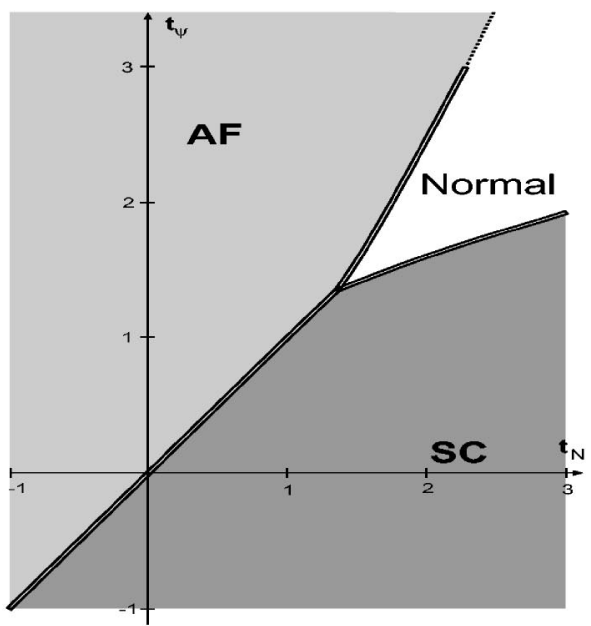

(b)

FIG. 6. Phase diagram of Eq. (29) with $\tilde{u}_{2}<0$ in three dimensions in the large $N$ limit including fluctuations. Parameters are $\tilde{u}_{1}=1 / N$ $\tilde{u}_{2}=-1 / 2 N$.

energy in Eq. (29). In writing Eq. (37) we used that in the large $N$ limit, $\tilde{u}_{\{1,2\}} \sim 1 / N, \sigma \sim \sqrt{N}$, and we neglected terms of the order of $1 / N$, including contributions from longitudinal fluctuations. A requirement of the cancellation of tadpole diagrams for $A_{L}$ gives the condition $r_{A}=0$, as one would expect from the Goldstone theorem. It is convenient to define parameter $r_{c}$ from the condition

$$
0=r_{c}+\left(12 \tilde{u}_{1}+4 \tilde{u}_{2}\right) N \int_{0}^{\Lambda} \frac{d^{3} k}{(2 \pi)^{3}} \frac{1}{k^{2}} .
$$

If we measure $r$ 's with respect to $r_{c}$

$$
\begin{gathered}
t_{\psi}=r_{1}-r_{c} \\
t_{N}=r_{2}-r_{c},
\end{gathered}
$$

we can absorb all the cutoff dependence of Eq. (37) into definitions of $t_{\psi}$ and $t_{N}$ :

$$
\begin{aligned}
0=t_{\psi}+ & 4\left(\tilde{u}_{1}+\tilde{u}_{2}\right) \sigma^{2}+4 \tilde{u}_{1} N \int \frac{d^{3} k}{(2 \pi)^{3}}\left(\frac{1}{k^{2}}-\frac{1}{k^{2}+r_{B}}\right) \\
r_{B}= & t_{\psi}+4 \tilde{u}_{1} \sigma^{2}+4\left(\tilde{u}_{1}+\tilde{u}_{2}\right) N \int\left(\frac{1}{k^{2}}-\frac{1}{k^{2}+r_{B}}\right) \\
& +4 \tilde{u}_{1} N \int \frac{d^{3} k}{(2 \pi)^{3}}\left(\frac{1}{k^{2}}-\frac{1}{k^{2}+r_{N}}\right) \\
r_{N}= & t_{N}+4 \tilde{u}_{1} \sigma^{2}+4 \tilde{u}_{1} N \int \frac{d^{3} k}{(2 \pi)^{3}}\left(\frac{1}{k^{2}}-\frac{1}{k^{2}+r_{B}}\right) \\
& +4\left(\tilde{u}_{1}+\tilde{u}_{2}\right) N \int\left(\frac{1}{k^{2}}-\frac{1}{k^{2}+r_{N}}\right) .
\end{aligned}
$$

Integrals are now convergent for large $k$, so upper limits of integration can be extended to infinity. Solutions to Eq. (40) correspond to extremal points of free energy as a function of $\sigma$. When both $t_{\psi}$ and $t_{N}$ are large, there are no solutions to
Eq. (40). This is a disordered phase. Once we decrease $t_{\psi}$ sufficiently, a single solution appears at $t_{\psi, M}$ and splits into two for $t_{\psi}<t_{\psi, M}$. This describes the appearance of the TSC phase as a locally stable state. The point $t_{\psi, M}$, where two solutions merge into one and disappear, correspond to the boundary of the local stability region of the TSC phase. As $t_{\psi}$ is lowered further, at a temperature $t_{\psi, L}$ one of the solutions approaches $\sigma=0$ and then disappears. This is a spinodal point below which a disordered phase is unstable to developing TSC order parameter. The actual first order phase transition occurs somewhere between $t_{\psi, M}$ and $t_{\psi, L}$.

Figure 6 shows a phase diagram constructed from the arguments presented above, for both TSC and AF phases. We note that the mixed phase with simultaneous TSC and AF orders is only possible on the $t_{\psi}=t_{N}$ line. Thus, the first order phase transition between two types of ordered phases remains even when we include fluctuations.

The most interesting feature of this phase diagram is that the transition between the disordered and the antiferromagnetic phases becomes first order in the vicinity of the critical point.

\section{FINITE TEMPERATURE ANALYSIS: NONUNITARY CASE}

Mean-field calculations for the free energy Eq. (29) with $\tilde{u}_{2}>0$ demonstrated that the TSC phase is nonunitary and there is a mixed phase with both TSC and AF order (see Sec. III and Fig. 4). Within mean field theory, the mixed phase terminates at a tetracritical point with $\mathrm{SO}(4)=\mathrm{SO}(3)$ $\times \mathrm{SO}(3)$ symmetry. The goal of this section is to examine how the tetracritical point is affected by thermal fluctuations.

To this end we extend the $\mathrm{SO}(3)$ spin symmetry to $\mathrm{SO}(N)$ and approach the problem with three different methods: A large $N$ analysis, a renormalization group calculation in $d$ $=4-\epsilon$ and in one in $d=2+\epsilon$. The physical image that emerges from all of these approaches is that a $\mathrm{SO}(3)$ $\times \mathrm{SO}(N)$ critical point exists for sufficiently large $N$, but 


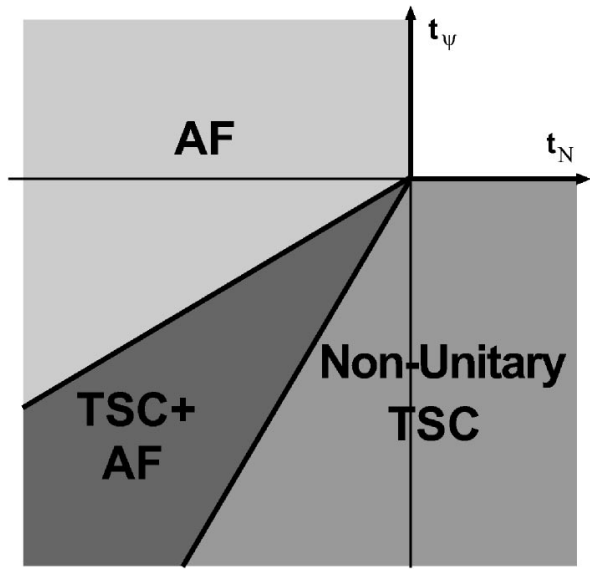

FIG. 7. Phase diagram of the model Eq. (29) with $\widetilde{u}_{2}>0$ in three dimensions in the large $N$ limit. The four second order lines meet at the tetracritical point at nonzero angles. The same phase diagram appears in the $d=4-\varepsilon$ analysis for $N \geqslant 33$ and in the $d=2+\varepsilon$ analysis for $N \geqslant 5$.

probably does not survive down to the physical $N=3$. We argue that in this case the tetracritical point is stretched to a line of direct first order transition from the normal state to the mixed phase.

\section{A. Large $N$ phase diagram in three dimensions}

We consider the model Eq. (29) in three dimensions for large $N$ and with $\tilde{u}_{2}>0$. We note that the quartic terms give a free energy that is bounded from below for $\tilde{u}_{1}+\tilde{u}_{2} / 3>0$. Thus, in this section we will always assume that this condition is satisfied. In Appendix $\mathrm{C}$ we also discuss that when $\tilde{u}_{1}+\tilde{u}_{2} / 3$ becomes small, of the order of $1 / N^{2}$ (in the large $N$ limit both $\tilde{u}$ s are of the order of $1 / N$ ), the phase diagram may change qualitatively.

In the mixed phase the TSC and AF both have nonzero expectation values and are orthogonal to each other. Hence, in the ordered phase we can choose

$$
\begin{aligned}
\langle\boldsymbol{\Psi}\rangle & =\left(0, \ldots, 0, \sigma_{\psi}, i \sigma_{\psi}, 0\right) \\
\langle\mathbf{N}\rangle & =\left(0, \ldots, 0,0,0, \sigma_{N}\right) .
\end{aligned}
$$

Following the discussion in Sec. V we introduce longitudinal and transverse fluctuations for all order parameters. It is easy to verify that the requirement of cancellation of tadpole diagrams for longitudinal components implies zero effective masses for the transverse components. Shifting $r_{1}$ and $r_{2}$ as in Eq. (39) we obtain self-consistency conditions for expectation values of the order parameters

$$
\begin{aligned}
& t_{\psi}+\left(12 \tilde{u}_{1}+4 \tilde{u}_{2}\right) \sigma_{\psi}^{2}+4 \tilde{u}_{1} \sigma_{N}^{2}=0 \\
& t_{\psi}+\left(4 \tilde{u}_{1}+4 \tilde{u}_{2}\right) \sigma_{N}^{2}+8 \tilde{u}_{1} \sigma_{\psi}^{2}=0 .
\end{aligned}
$$

These equations can be easily solved and we obtain a phase diagram shown in Fig. 7.

We observe that in this case the only effect of fluctuations is to shift the tetracritical point from $r_{1}=r_{2}=0$ to $r_{1}=r_{2}=r_{c}$.

\section{B. Renormalization group analysis in $d=4-\epsilon$. $\operatorname{SO}(3) \times \operatorname{SO}(N)$ fixed point}

As shown in Fig. 5 for $N=3$, all fixed points with symmetry $\mathrm{SO}(4) \sim \mathrm{SO}(3) \times \mathrm{SO}(3)$ are unstable within a $4-\epsilon$ expansion. In contrast, as $N$ is increased a fixed point with $\mathrm{SO}(3) \times \mathrm{SO}(N)$ symmetry appears that is fully stable with respect to changes in the quartic interaction parameters, including those perturbations that destroy $\mathrm{SO}(3) \times \mathrm{SO}(N)$ symmetry. Such fixed point exists for $N \geqslant 33$ and has

$$
\begin{gathered}
v_{1}=-\frac{3 \epsilon}{2 K_{d}} h(N) \\
v_{2}=\frac{\epsilon}{4 K_{d}} \frac{1-72 h(N)}{N+7} \\
r_{1}=r_{2}=-2 K_{d}\left[(3 N+2) v_{1}+(N+6) v_{2}\right],
\end{gathered}
$$

where $u_{1}, u_{2}$, and $u_{3}$ are related to $v_{1}$ and $v_{2}$ by the constraints (31), and the function $h(N)=\left[N^{2}+8 N-65+(N\right.$ $\left.+7) \sqrt{N^{2}-34 N+49}\right]^{-1} \sim 1 / 2 N^{2}+O\left(1 / N^{3}\right)$ is real only for $N>32$.

The two quadratic parameters $r_{1}$ and $r_{2}$ are relevant, tuning the transition on a two-dimensional phase diagram. The RG flow Eq. (34), linearized about the fixed point Eq. (43), yield two principal directions, $\left(\delta r_{1}, \delta r_{2}\right) \propto(1,1)$ associated with the thermal exponent $\lambda_{t}$, and $\left(\delta r_{1}, \delta r_{2}\right) \propto(-1,2)$ associated with the anisotropy exponent $\lambda_{g}$. From these we find the critical exponents

$$
\begin{gathered}
1 / \nu=\lambda_{t}=2-\epsilon\left(1-\frac{10}{N}+\frac{28}{N^{2}}\right)+O\left(\frac{\epsilon}{N^{3}}\right), \\
\phi=\lambda_{g} \nu=1-\epsilon\left(\frac{3}{2 N}+\frac{51}{2 N^{2}}\right)+O\left(\frac{\epsilon}{N^{3}}\right) .
\end{gathered}
$$

Note that the crossover exponent $\phi$ for the anisotropy is always less than one. This implies ${ }^{71}$ that the phase boundaries meet as straight lines at the critical point, and we find the same topology of the phase diagram as shown in Fig. 7.

\section{Renormalization group analysis in $d=2+\epsilon$. $\operatorname{SO}(3) \times \operatorname{SO}(N)$ fixed point}

The runaway flows in Eq. (34) mean that the system goes to strong coupling. In this limit the magnitudes of the vectors $\operatorname{Re} \boldsymbol{\psi}, \operatorname{Im} \psi$, and $\mathbf{N}$ have already developed locally but the directions can still fluctuate on long length scales. For $N=3$ one of the runaway directions of Eq. (34) corresponds to $u_{3}$, $v_{2}>0$ and $u_{1}, v_{1}<0$. The corresponding strong coupling limit can be described by a triad of vectors that are all mutually orthogonal.

$$
F=-\sum_{\langle x y\rangle}\left\{K_{1} \mathbf{e}_{1}(x) \cdot \mathbf{e}_{1}(y)+K_{2}\left[\mathbf{e}_{2}(x) \cdot \mathbf{e}_{2}(y)+\mathbf{e}_{3}(x) \cdot \mathbf{e}_{3}(y)\right]\right\} .
$$

Here $\mathbf{e}_{1}, \mathbf{e}_{2}$, and $\mathbf{e}_{3}$ correspond to $\mathbf{N}, \operatorname{Re} \boldsymbol{\Psi}$, and $\operatorname{Im} \boldsymbol{\Psi}$, respectively. The free energy Eq. (45) has an explicit $\mathrm{SO}(2)$ 


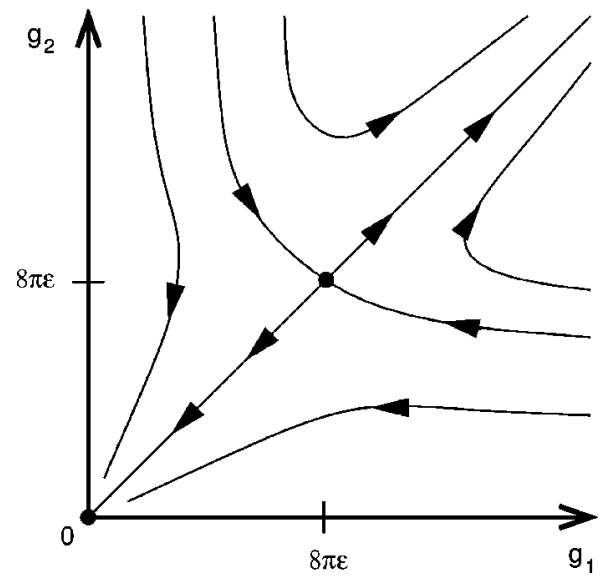

FIG. 8. Renormalization group flow of nonlinear model Eq. (46), corresponding to Eq. (47).

charge symmetry of rotations between $\mathbf{e}_{2}$ and $\mathbf{e}_{3}$. The continuum version of this model is given by

$$
F=\int d^{d} x\left(\frac{1}{2 g_{1}}\left(\nabla \mathbf{e}_{1}\right)^{2}+\frac{1}{2 g_{2}}\left[\left(\nabla \mathbf{e}_{2}\right)^{2}+\left(\nabla \mathbf{e}_{3}\right)^{2}\right]\right),
$$

where $g_{i} \propto K_{i}^{-1}$ and the constraints $\mathbf{e}_{i} \cdot \mathbf{e}_{j}=\delta_{i j}$ are implied.

Let us now discuss the phase diagram of Eqs. (45) and (46). For $K_{1,2} \rightarrow 0$ we have a fully disordered phase. For $K_{1,2} \rightarrow \infty$ we have a fully ordered phase that is a mixture of TSC and AF. When $K_{2}=\infty$ the vectors $\mathbf{e}_{1}$ and $\mathbf{e}_{2}$ are ordered and there is an Ising type transition between TSC and TSC + AF phases. For $K_{1}=\infty$ vector $\mathbf{N}$ is ordered and there is an $\mathrm{O}(2)$ transition between the AF and TSC+AF states. For $K_{2}=0$ there is a Heisenberg transition between the disordered and the AF phases. For $K_{3}=0$ there is a transition between the fully disordered and the TSC phases. What happens in the interior of the phase diagram, however, is not clear.

When we apply the $d-2=\epsilon \mathrm{RG}$ analysis to the model Eq. $(46)^{75-79}$ we obtain the flow equations

$$
\begin{gathered}
\frac{d g_{1}}{d l}=-\epsilon g_{1}+\frac{g_{1}^{2}\left(g_{2}^{2}+g_{1} g_{2}-g_{1}^{2}\right)}{2 \pi\left(g_{1}+g_{2}\right)^{2}} \\
\frac{d g_{2}}{d l}=-\epsilon g_{2}+\frac{g_{1}^{2} g_{2}^{2}}{2 \pi\left(g_{1}+g_{2}\right)^{2}} .
\end{gathered}
$$

The flow diagram is shown in Fig. 8 .

We can see that it lacks the Ising and $\mathrm{O}(2)$ phase transitions. This is not surprising, since the $d-2=\epsilon$ analysis works well only for the spin-wave excitations of order parameters with $N \geqslant 3$.

To shed some light on the phase diagram of Eqs. (45) and (46) we consider the large $N$ generalization of this model. We assume that all vectors $\mathbf{e}_{i}$ have $N$ components

$$
\begin{aligned}
F= & \int d^{d} x\left[\frac{1}{2 g_{1}}\left(\nabla \mathbf{e}_{1}\right)^{2}+\frac{1}{2 g_{2}}\left[\left(\nabla \mathbf{e}_{2}\right)^{2}+\left(\nabla \mathbf{e}_{3}\right)^{2}\right]\right. \\
& \left.+\frac{1}{g_{3}}\left[\left(\mathbf{e}_{1} \cdot \nabla \mathbf{e}_{2}\right)^{2}+\left(\mathbf{e}_{1} \cdot \nabla \mathbf{e}_{3}\right)^{2}\right]+\frac{1}{g_{4}}\left(\mathbf{e}_{2} \cdot \nabla \mathbf{e}_{3}\right)^{2}\right]
\end{aligned}
$$

The last two terms in Eq. (48) are generated in the RG flow, even if they are absent in the microscopic model (such terms are linearly independent of the first two only for $N>3$ ). The symmetry breaking pattern of the nonlinear model Eq. (48) is

$$
\mathrm{O}(N) \times \mathrm{O}(2) / \mathrm{O}(N-3) \times \mathrm{O}(2)_{\mathrm{diag}} .
$$

In order to express the RG equations in a simple form we introduce the variables $\eta_{i}$ :

$$
\begin{gathered}
\eta_{1}=\frac{1}{g_{1}}, \\
\eta_{2}=\frac{1}{g_{2}}, \\
\eta_{3}=\frac{1}{g_{1}}+\frac{1}{g_{2}}+\frac{2}{g_{3}}, \\
\eta_{4}=\frac{2}{g_{2}}+\frac{2}{g_{4}} .
\end{gathered}
$$

These variables arise naturally in a matrix formulation of the nonlinear model Eq. (48), see Appendix D, where the RG calculation is outlined. To one loop order we find the RG flow

$$
\begin{gathered}
\frac{d \eta_{1}}{d l}=\epsilon \eta_{1}-\frac{1}{2 \pi}\left(N-2+\frac{\eta_{1}^{2}-\eta_{2}^{2}-\eta_{3}^{2}}{\eta_{2} \eta_{3}}\right) \\
\frac{d \eta_{2}}{d l}=\epsilon \eta_{2}-\frac{1}{2 \pi}\left(N-2+\frac{\eta_{2}^{2}-\eta_{3}^{2}-\eta_{1}^{2}}{2 \eta_{1} \eta_{3}}-\frac{\eta_{4}}{2 \eta_{2}}\right) \\
\frac{d \eta_{3}}{d l}=\epsilon \eta_{3}-\frac{1}{2 \pi}\left(N-2+\frac{N-3}{2} \frac{\eta_{3}^{2}-\eta_{1}^{2}-\eta_{2}^{2}}{\eta_{1} \eta_{2}}-\frac{\eta_{4}}{2 \eta_{3}}\right) \\
\frac{d \eta_{4}}{d l}=\epsilon \eta_{4}-\frac{1}{2 \pi}\left(\frac{N-3}{2} \frac{\eta_{4}^{2}}{\eta_{2}^{2}}+\frac{\eta_{4}^{2}}{2 \eta_{3}^{2}}\right) .
\end{gathered}
$$

The conditions $g_{1}=g_{2}$ and $g_{3}=g_{4}$ define a twodimensional subspace over which the free energy Eq. (48) has the enhanced symmetry $\mathrm{SO}(3) \times \mathrm{SO}(N)$. For arbitrary $N$, the $\mathrm{RG}$ equations (51) have $\mathrm{SO}(3) \times \mathrm{SO}(N)$ fixed point

$$
\begin{gathered}
\eta_{1}=\eta_{2}=(N-2-x) / \epsilon, \\
\eta_{3}=\eta_{4}=x \eta_{1},
\end{gathered}
$$

where $x=\left(N-2+\sqrt{N^{2}-5 N+5}\right) /(N-1)$. Independent of $N$, this point has one stable direction and one unstable direction within the symmetric plane. The flow in directions perpendicular to the symmetric plane depends on the value of $N$. 
The case $N=4$ is special and is discussed in Appendix D. For all other $N \geqslant 5$, the RG flow away from the $\mathrm{SO}(3) \times \mathrm{SO}(N)$ plane has one stable and one unstable direction. Two relevant parameters are therefore necessary to tune the transition, just as we found in the $d=4-\epsilon$ analysis for large $N$, Eq. (43). Computing the critical exponents $\nu$ and $\phi$, just as we did in the case $d=4-\epsilon$, Eq. (44), we find,

$$
\begin{gathered}
1 / \nu=\epsilon, \\
\phi=1-\frac{63}{16 N^{2}}-\mathrm{O}\left(\frac{1}{N^{3}}\right) .
\end{gathered}
$$

Just as before, we find that for all finite $N, \phi$ is less than one, leading to a phase diagram that is topologically equivalent to that found in large $N$ expansion and in $4-\epsilon \mathrm{RG}$ analysis (see Fig. 7). What is more, to leading order in $1 / N$, expansions about the upper and lower critical dimension, Eqs. (44) and (53), lead to the same critical exponents,

$$
\begin{gathered}
1 / \nu=d-2+\mathrm{O}\left(\frac{1}{N}\right) \\
\phi=1-\mathrm{O}\left(\frac{1}{N}\right) .
\end{gathered}
$$

This supports the fact that for large $N$ the $\mathrm{SO}(3) \times \mathrm{SO}(N)$ fixed point changes adiabatically with dimension.

\section{Phase diagram for $N=3$ in three dimensions}

We employed three approaches to study classical fluctuations in systems with competing $\mathrm{AF}$ and nonunitary TSC: large $N$ expansion, $d=4-\epsilon$ and $d=2+\epsilon \mathrm{RG}$ analyses. When $N$ is large all three consistently predict a tetracritical point with enhanced $\mathrm{SO}(3) \times \mathrm{SO}(N)$ symmetry. In the physically relevant case $N=3$ and $d=3$ the situation is less clear. For example, expansion from the upper critical dimension $(d$ $=4-\epsilon$ ) shows that such fixed point appears only for $N \geqslant 33$. Expansion from the lower critical dimension $(d=2+\epsilon)$ gives a $\mathrm{SO}(3) \times \mathrm{SO}(N)$ fixed point in the RG flow for any $N \geqslant 3$, but these fixed points become tetracritical points on the phase diagram only for $N \geqslant 5$. It is possible that in three dimensions even for $N=3$ there is a tetracritical $\mathrm{SO}(3)$ $\times \mathrm{SO}(3)$ point. The reason why perturbative expansions in dimension fail to see it, is that they work well for small $\epsilon$ and extrapolations to $d=3$ should be treated with caution. ${ }^{79}$ Such a scenario, however, would contradict the results of classical Monte Carlo simulations in Ref. 80, in which the model (45) has been analyzed for $K_{1}=K_{2}$. In that paper the bimodal distribution in the energy histogram has been interpreted as a signature of the first order transition.

The phase diagram that we propose for the model Eq. (45) and for systems with competing AF and nonunitary TSC in general is shown in Fig. 9. Thermal fluctuations turn a tetracritical point into a line of direct first-order transitions between a disordered and a mixed TSC/AF phase. We expect, however, that the width of such a first order line is small and the transition is very weakly first order. We conjecture that

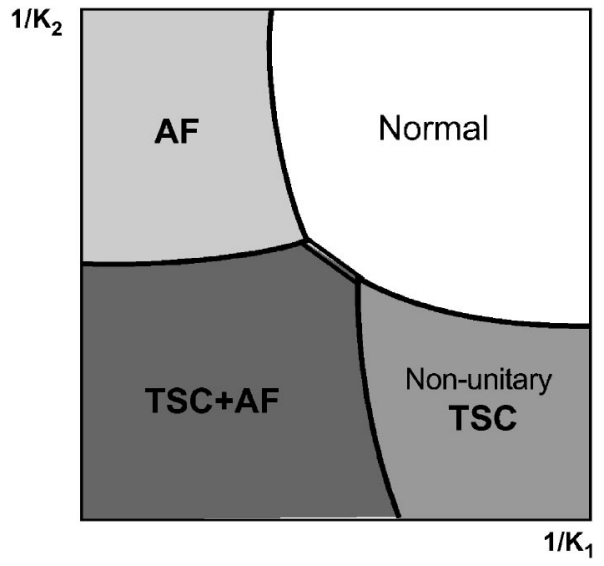

FIG. 9. Phase diagram for systems with competing AF and nonunitary TSC orders described by the model Eq. (45) in three dimensions. The tetracritical point in the mean-field phase diagram of the GL free energy in Eq. (29) (see Fig. 4) is replaced by a line of direct first order transitions between a disordered and a mixed TSC/AF phase.

when approaching the transition between the normal and the AF/TSC mixed phase, susceptibilities for the AF and TSC order parameters start increasing as if dominated by the $\mathrm{SO}(3) \times \mathrm{SO}$ (3) tetracritical point. Only very close to the transition line the divergencies are cutoff due to the transition being first order.

Finally, we note that Eq. (45) with $K_{1}=0$ is among a class of closely related models that have been studied extensively in the context of frustrated magnetism. ${ }^{81-91}$ Numerical studies of these models in $d=3$ dimensions yield nonuniversal critical properties at the boundary of normal and TSC phases, ${ }^{82-90}$ and even evidence of a first order transition. ${ }^{91}$ The nonperturbative theoretical analysis of Ref. 92 supports the latter scenario, claiming that the critical point observed in nonlinear sigma models in $d=2+\epsilon$ disappears at $d_{c}=2.87$ in one such model, being replaced by a weakly first order transition above $d_{c}$. The exact nature of the transition seems to be very strongly model dependent near $d=3$, and we leave open the possibility that the transition between nonunitary TSC and normal phases is weakly first order.

\section{QUANTUM SO(4) SYMMETRY}

The microscopic system that motivated our discussion is an assembly of Luttinger liquids weakly coupled in three dimensions. It is useful to condense this system to a simpler effective quantum model that concentrates on the low energy collective degrees of freedom, such as AF and TSC order parameters and rotations between them (such a description only applies in the vicinity of the AF/TSC phase boundary shown in Fig. 1). Effective quantum models have been discussed previously for spin systems (see Ref. 93 for a review), and systems with singlet superconductivity competing either with charge density wave $\operatorname{order}^{37,38,65,94}$ or with antiferromagnetism. ${ }^{30,95-98}$

A simple form for such an effective model is a $\mathrm{SO}(4)$ quantum rotor model: 


$$
\begin{aligned}
\mathcal{H}_{\mathrm{r}}= & \frac{1}{2 \chi_{1}} \sum_{i} \mathbf{S}_{i}^{2}+\frac{1}{2 \chi_{2}} \sum_{i} \mathbf{I}_{i}^{2}-J \sum_{\langle i j\rangle a \alpha} Q_{i, a \alpha} Q_{j, a \alpha} \\
& +\widetilde{u}_{1} \sum_{i a b \alpha \beta} Q_{i, a \alpha}^{2} Q_{i, b \beta}^{2}+\widetilde{u}_{2} \sum_{i a b \alpha \beta} Q_{i, a \alpha} Q_{i, a \beta} Q_{i, b \alpha} Q_{i, b \beta} \\
& +\delta r \sum_{i \alpha}\left(Q_{i, z \alpha}^{2}-Q_{i, x \alpha}^{2}-Q_{i, y \alpha}^{2}\right) .
\end{aligned}
$$

The model is obtained by coarse graining the original lattice so that, for a half-filled system, each site of the rotor model includes two (or a larger even number, as necessary to include an integer number of spin-triplet Cooper pairs) adjacent sites along the intrachain direction of the original lattice. By combining the electronic operators that make up each rotor model site, one can build three local spin and three local isospin operators $\mathbf{S}_{i}$ and $\mathbf{I}_{i}$, and a $\mathrm{SO}(4)$ tensor order parameter $Q_{i, a \alpha}$. Following a procedure similar to Ref. 97, one can show that the low energy properties of the system are given by the rotor commutation relations:

$$
\begin{gathered}
{\left[S_{i, \alpha}, S_{j, \beta}\right]=i \delta_{i j} \epsilon_{\alpha \beta \gamma} S_{i, \gamma}} \\
{\left[I_{i, a}, I_{j, b}\right]=i \delta_{i j} \epsilon_{a b c} I_{i, c}} \\
{\left[S_{i, \alpha}, Q_{j, a \beta}\right]=i \delta_{i j} \epsilon_{\alpha \beta \gamma} Q_{j, a \gamma}} \\
{\left[I_{i, a}, Q_{j, b \alpha}\right]=i \delta_{i j} \epsilon_{a b c} Q_{j, c \alpha}} \\
{\left[Q_{i, a \alpha}, Q_{j, b \beta}\right]=0 .}
\end{gathered}
$$

These relations are analogous to Eqs. (4), (7), and (19). In Eq. (55) the unit length constraint of the rigid rotor models is replaced by the interaction terms $\widetilde{u}_{1}$ and $\widetilde{u}_{2}$. For $\delta r$ negative the system favors the AF state and for $\delta r$ positive the TSC state is preferred. When $\delta r=0$ all generators of $\mathrm{SO}(4)$ (I $=\sum_{i} \mathbf{I}_{i}$ and $\mathbf{S}=\sum_{i} \mathbf{S}_{i}$ ) commute with the Hamiltonian Eq. (55) and the system is exactly $\mathrm{SO}(4)$ symmetric.

We can use Eq. (55) to discuss excitation spectra in various phases of the system. We choose to orient the AF order parameter $\left(Q_{z \alpha}\right)$ in the $z$ direction so that $\left\langle Q_{z z}\right\rangle=N$. Similarly we take $\left\langle Q_{x x}\right\rangle=\psi$ to describe unitary TSC, and $\left\langle Q_{x x}\right\rangle=\left\langle Q_{y y}\right\rangle$ $=\psi$. for nonunitary TSC. With these choices we can linearize the equations of motion for the fluctuations to obtain:

$$
\frac{d Q_{j, b \beta}}{d t}=-\frac{1}{\chi_{1}} \sum_{\alpha} S_{j, \alpha} \epsilon_{\alpha \beta b}\left\langle Q_{b b}\right\rangle-\frac{1}{\chi_{2}} \sum_{\alpha} I_{j, \alpha} \epsilon_{\alpha b \beta}\left\langle Q_{\beta \beta}\right\rangle,
$$

$$
\begin{aligned}
\frac{d S_{j, \alpha}}{d t}= & \frac{J}{2} \sum_{\beta^{\prime}, b^{\prime}} \epsilon_{\alpha \beta^{\prime} b^{\prime}}\left\langle Q_{b^{\prime} b^{\prime}}\right\rangle \sum_{\delta}\left(Q_{j, b^{\prime} \beta^{\prime}}-Q_{j, b^{\prime} \beta^{\prime}+\delta}\right), \\
\frac{d I_{j, a}}{d t}= & \frac{J}{2} \sum_{\beta^{\prime}, b^{\prime}} \epsilon_{a \beta^{\prime} b^{\prime}}\left\langle Q_{\beta^{\prime} \beta^{\prime}}\right\rangle \sum_{\delta}\left(Q_{j, b^{\prime} \beta^{\prime}}-Q_{j, b^{\prime} \beta^{\prime}+\delta}\right) \\
& +4 \delta r\left[\epsilon_{a z \beta^{\prime}}\left\langle Q_{\beta^{\prime} \beta^{\prime}}\right\rangle Q_{j, z \beta^{\prime}}+\epsilon_{a z b^{\prime}} Q_{j, b^{\prime} z}\left\langle Q_{z z}\right\rangle\right] .
\end{aligned}
$$

The above equations define a linear eigenvalue problem for the frequencies of the collective modes and for the second quantized operators

$$
b^{\dagger}(\mathbf{k})=\sum_{c \neq \gamma} A_{c \gamma}^{1} Q_{c \gamma}(\mathbf{k})+\sum_{a} A_{\alpha}^{2} S_{\alpha}(\mathbf{k})+\sum_{a} A_{a}^{3} I_{a}(\mathbf{k}),
$$

which obey $\dot{b}^{\dagger}(\mathbf{k})=i\left[H, b_{\mathbf{k}}^{\dagger}\right]=i \omega_{\mathbf{k}} b^{\dagger}(\mathbf{k})$. The Fourier transforms of lattice operators are defined by $\hat{O}(\mathbf{k})$ $=N^{-1 / 2} \Sigma_{j} \hat{O}_{j} e^{i \mathbf{k} \cdot \mathbf{x}_{j}}$. In the effective model, neutrons couple to the spin order parameter $Q_{z \alpha}$, so that the low energy scattering intensity of polarized neutrons is given by:

$$
\chi_{\alpha}^{\prime \prime}\left(\mathbf{k}+2 \mathbf{k}_{f}, \omega\right)=\sum_{n}\left|\left\langle n\left|Q_{z \alpha}(\mathbf{k})\right| 0\right\rangle\right|^{2} \delta\left(\omega-\omega_{n 0}\right) .
$$

The momentum is shifted by $2 k_{f}$ because a uniform $Q_{z \alpha}$ in Eq. (55) corresponds to a SDW order of momentum $2 k_{f}$ in the microscopic model. The weight associated with a particular collective mode created by $b^{\dagger}(\mathbf{k})$ is

$$
\begin{aligned}
& \left|\left\langle 0\left|b(\mathbf{k}) Q_{z \alpha}(\mathbf{k})\right| 0\right\rangle\right|^{2} \delta\left(\omega-\omega_{\mathbf{k}}\right) \\
& \quad=\left|\left\langle 0\left|\left[b(\mathbf{k}), Q_{z \alpha}(\mathbf{k})\right]\right| 0\right\rangle\right|^{2} \delta\left(\omega-\omega_{\mathbf{k}}\right),
\end{aligned}
$$

where we used the fact that $b(\mathbf{k})$ annihilates the ground state. The commutator can be calculated using Eq. (56), once the operator content of the mode $b(\mathbf{k})$ is determined. We note that for $\mathbf{k}$ close to zero, neutrons couple to $S_{\alpha}$ instead of $Q_{z \alpha}$, in which case,

$$
\chi_{\alpha}^{\prime \prime}(\mathbf{k}, \omega)=\sum_{n}\left|\left\langle n\left|S_{\alpha}(\mathbf{k})\right| 0\right\rangle\right|^{2} \delta\left(\omega-\omega_{n 0}\right) .
$$

However, in the following, we focus on neutron scattering near $2 k_{f}$.

The nature of collective excitations in the various phases is summarized in Figs. 10-12, and Table I. We now provide a detailed analysis of the collective mode spectrum and the associated neutron scattering intensity in each phase. A complementary calculation of the neutron scattering intensity of the $\Theta$-excitations in the unitary TSC, based on the microscopic model, is given in Ref. 55.

\section{A. Antiferromagnet}

In the AF phase $\left\langle Q_{z z}\right\rangle=N$ and all other order parameters vanish. Then Eqs. (57)-(59) decouple to four independent collective modes. The equations of motion for the pairs $\left\{Q_{z x}, S_{y}\right\}$ and $\left\{Q_{z y}, S_{x}\right\}$ yield the usual AF spin waves with linear dispersion reflecting broken $\mathrm{SO}(3)$ spin symmetry:

$$
\omega_{\mathrm{AF}, S}\left(\mathbf{k}+2 \mathbf{k}_{f}\right)=N \sqrt{\frac{J z}{2 \chi_{1}}\left(1-\gamma_{\mathbf{k}}\right)} \approx \sqrt{\frac{J}{2 \chi_{1}}}|\mathbf{k}|,
$$

where $J_{k}=J z /\left(2 \chi_{1}\right)\left(1-\gamma_{\mathbf{k}}\right), z$ is the lattice coordination $(z$ $=6$ for a cubic lattice in three dimensions), $\gamma_{k}=z^{-1} \sum_{\delta} e^{i \mathbf{k} \delta}$, and $\boldsymbol{\delta}$ are bond vectors. Although $\mathbf{k}$ and $\mathbf{k}+2 \mathbf{k}_{f}$ are related by reciprocal lattice vectors in the rotor model Eq. (55), they are not in the microscopic Hamiltonian, and the addition of $2 \mathbf{k}_{f}$ to the argument of Eq. (64) serves as a mnemonic for the fact 
(a)

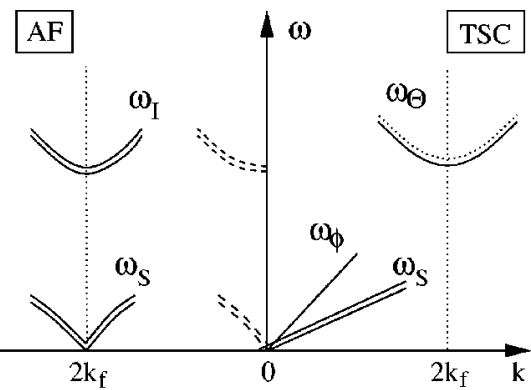

(b)

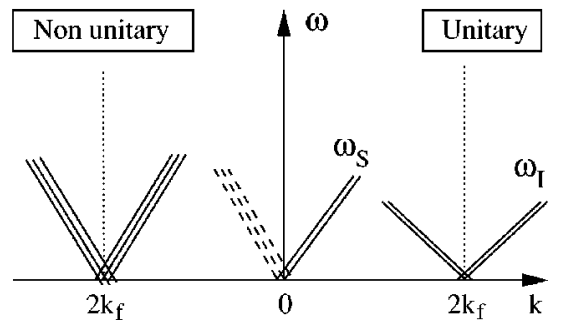

FIG. 10. Collective excitations in various phases: (a) In the AF phase, the excitation spectrum consists of two massless spin waves and two massive isospin waves. Due to translational symmetry breaking, $2 k_{f}$ is a reciprocal lattice vector, and these modes also have nonzero weight at the dashed curves near $k=0$. The spectrum in the TSC phase contains massless phase and spin modes, as well as massive $\Theta$ modes. For unitary TSC, there is only one such mode. The nonunitary TSC (as well as the mixed phase) contains a second, degenerate $\Theta$ mode, represented by the dotted curve; (b) the $\mathrm{SO}(4)$ symmetric point $(\delta r=0)$. In a unitary TSC this point corresponds to the transition between AF and TSC. It is characterized by four gapless (Goldstone) modes (two isospin and two spin). In the nonunitary case the $\mathrm{SO}(4)$ symmetric point is inside the mixed AF/TSC phase. It supports only three (degenerate) Goldstone modes. This is because the order parameter has a residual $\mathrm{SO}(3)$ symmetry.

that the spin mode is centered primarily around $2 \mathbf{k}_{f}$.

Similarly the equations of motion for $\left\{Q_{x z}, I_{y}\right\}$ and $\left\{Q_{y z}, I_{x}\right\}$ describe two massive isospin waves:

$$
\omega_{\mathrm{AF}, I}\left(\mathbf{k}+2 \mathbf{k}_{f}\right)=N \sqrt{\frac{4|\delta r|}{\chi_{2}}+\frac{J}{2 \chi_{2}} k^{2}} .
$$

These excitations correspond to rotations between the AF and the TSC states and indicate proximity of the two ground states. When $\delta r$ goes to zero, the mass of the isospin waves vanishes reflecting an enhanced $\mathrm{SO}(4)$ symmetry. The isospin modes in the AF phase do not couple to neutrons.

\section{B. Unitary triplet superconductor}

In the unitary $\operatorname{TSC}\left(\delta r>0, \tilde{u}_{2}<0\right)$ we choose $\left\langle Q_{x x}\right\rangle=\psi$, while all other order parameters vanish. Equations (57) and (58) for the pairs $\left\{Q_{x y}, S_{z}\right\}$ and $\left\{Q_{x z}, S_{y}\right\}$ yield two spin wave modes reflecting the broken spin symmetry:

$$
\omega_{\mathrm{uTSC}, S}(\mathbf{k})=\psi \sqrt{\frac{J}{2 \chi_{1}}}|\mathbf{k}| .
$$

The creation operators for the two spin waves involve the generators $S_{y}$ and $S_{z}$, respectively. Substitution into Eq. (62)
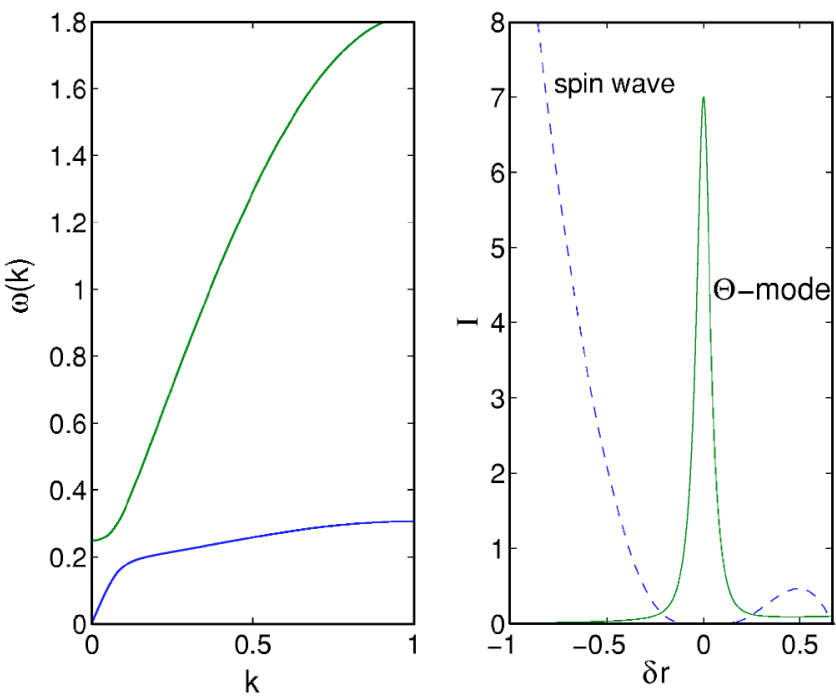

FIG. 11. Spin waves and $\Theta$ modes in the mixed phase: (a) dispersions $(\delta r>0)$ of the mode clearly show mixing between the $\Theta$ mode and the spin wave. Each of the shown modes is doubly degenerate; (b) neutron scattering intensity of the modes at wavevector $k=2 k_{f}+0.1 \pi$ as a function of the tuning parameter $\delta r$. Note that the weight of the spin wave modes goes to zero at the $\mathrm{SO}(4)$ symmetric point $\delta r=0 . \Theta$ modes are strongly enhanced near $\delta r=0$.

immediately shows that the neutron scattering weight of these modes at $2 \mathbf{k}_{f}$ vanishes.

Equations (57) and (59) for the pair $\left\{Q_{y x}, I_{z}\right\}$ yields a gapless phase fluctuation mode reflecting the broken charge $U(1)$ symmetry in the TSC phase:

$$
\omega_{\mathrm{uTSC}, \varphi}(\mathbf{k})=\psi \sqrt{\frac{J}{2 \chi_{2}}}|\mathbf{k}| .
$$

With the inclusion of Coulomb interactions, this mode becomes massive through the Higgs phenomenon, with a mass of the order of the plasma frequency. The creation operator of this isospin mode involves the generator $I_{z}$. Substitution into Eq. (62) shows that it does not couple to $2 k_{f}$ neutrons.

Finally the equations of motion for the pair $\left\{Q_{z x}, I_{y}\right\}$ give the massive $\Theta$ mode:

$$
\omega_{\mathrm{uTSC}, \Theta}\left(\mathbf{k}+2 \mathbf{k}_{f}\right)=\psi \sqrt{\frac{J}{2 \chi_{2}} k^{2}+\frac{4 \delta r}{\chi_{2}}} .
$$

The operator that creates this mode from the ground state is given by

$$
\begin{aligned}
b_{\Theta}^{\dagger}(\mathbf{k})= & \frac{1}{\sqrt{1+\chi_{2}\left(J k^{2} / 2+4 \delta r\right)}} \\
& \times\left[\sqrt{\chi_{2}\left(J k^{2} / 2+4 \delta r\right)} Q_{z x}(\mathbf{k})+i I_{y}(\mathbf{k})\right] .
\end{aligned}
$$

When substituted into Eq. (62) it gives a neutron scattering intensity at $\mathbf{k}+2 \mathbf{k}_{f}$ : 


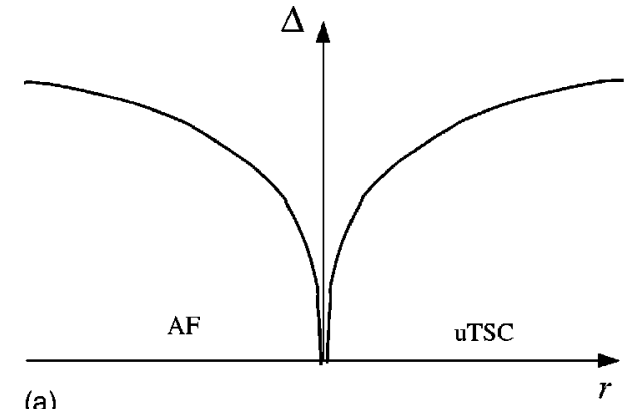

(a)

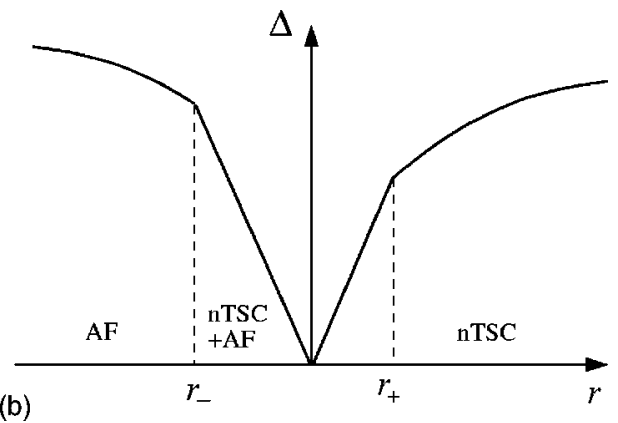

FIG. 12. Gap of the $\Theta$ modes softens toward $\delta r=0$ reflecting the enhanced $\mathrm{SO}(4)$ symmetry at that point: (a) The gap decreases as $\sqrt{|\delta r|}$ in the case $\tilde{u}_{2}<0$ (unitary TSC); (b) in the case $\widetilde{u}_{2}>0$ there is a change from $\sqrt{|\delta r|}$ behavior in the pure phases to linear decrease at smaller $|\delta r|$, inside the mixed nonunitary TSC and AF phase.

$$
\begin{aligned}
\left|\left\langle 0\left|\left[b_{\Theta}(\mathbf{k}), Q_{z \alpha}(\mathbf{k})\right]\right| 0\right\rangle\right|^{2} \delta\left(\omega-\omega_{\mathrm{uTSC}, \Theta}\right) \\
\quad=\delta_{\alpha x} \frac{\psi^{2}}{1+\chi_{2}\left(J k^{2} / 2+4 \delta r\right)} \delta\left[\omega-\omega_{\mathrm{nTSC}, \Theta}\left(\mathbf{k}+2 \mathbf{k}_{f}\right)\right] .
\end{aligned}
$$

As we approach the point $\delta r=0$ with $\mathrm{SO}(4)$ symmetry, the gap of the $\Theta$-mode vanishes as $\sqrt{\delta r}$. Hence, the spectrum of the Hamiltonian (55) with $\tilde{u}_{2}<0$ is such that on both sides of the AF/TSC transition we observe mode softening. Mode softening at the first order transition is a property of the higher symmetry quantum critical points..$^{30,94,95}$ Exactly at the $\mathrm{SO}(4)$ symmetric point $\delta r=0$ the system has gapless spin and isospin wave doublets.

\section{Nonunitary triplet superconductor}

For the case $\tilde{u}_{2}>0$ the TSC phase is nonunitary. We choose $\left\langle Q_{x x}\right\rangle=\left\langle Q_{y y}\right\rangle=\psi$. There is also a mixed phase where a nonvanishing AF order parameter $\left\langle Q_{z z}\right\rangle=N$ appears in addition to the nonunitary TSC order, considered below. In the pure nonunitary TSC we find two spin wave modes $\left\{Q_{x z}, S_{y}\right\}$ and $\left\{Q_{y z}, S_{x}\right\}$ with linear dispersion:

$$
\omega_{\mathrm{nTSC}, S}(\mathbf{k})=\psi \sqrt{\frac{J}{2 \chi_{1}}}|\mathbf{k}| .
$$

As in the unitary case, these spin waves do not couple to neutrons around $2 \mathbf{k}_{f}$.
The nonunitary TSC also supports two degenerate massive $\Theta$-modes $\left\{Q_{z x}, I_{y}\right\}$ and $\left\{Q_{z y}, I_{x}\right\}$ with dispersion

$$
\omega_{n \mathrm{TSC}, \Theta}\left(\mathbf{k}+2 \mathbf{k}_{f}\right)=\psi \sqrt{\frac{J}{2 \chi_{2}} k^{2}+\frac{4 \delta r}{\chi_{2}}} .
$$

These correspond to rotations of the real and the imaginary parts of the TSC order parameter toward the AF. These excitations are created by the operators:

$$
\begin{aligned}
b_{\Theta_{x}}^{\dagger}(\mathbf{k})= & \frac{1}{\sqrt{1+\chi_{2}\left(J k^{2} / 2+4 \delta r\right)}} \\
& \times\left[\sqrt{\chi_{2}\left(J k^{2} / 2+4 \delta r\right)} Q_{z y}(\mathbf{k})-i I_{x}(\mathbf{k})\right]
\end{aligned}
$$

$$
\begin{aligned}
b_{\Theta_{y}}^{\dagger}(\mathbf{k})= & \frac{1}{\sqrt{1+\chi_{2}\left(J k^{2} / 2+4 \delta r\right)}} \\
& \times\left[\sqrt{\chi_{2}\left(J k^{2} / 2+4 \delta r\right)} Q_{z x}(\mathbf{k})+i I_{y}(\mathbf{k})\right] .
\end{aligned}
$$

Substituting the $b_{\Theta_{\alpha}}$ operators in Eq. (62) we find the neutron scattering weight near $2 \mathbf{k}_{f}$ :

$$
\begin{aligned}
\mid\langle 0| & {\left.\left[b_{\Theta}(\mathbf{k}), Q_{z \alpha}(\mathbf{k})\right]|0\rangle\right|^{2} \delta\left(\omega-\omega_{\mathrm{nTSC}, \Theta}\right) } \\
& =\delta_{\alpha y} \frac{\psi^{2}}{1+\chi_{2}\left(J k^{2} / 2+4 \delta r\right)} \delta\left[\omega-\omega_{\mathrm{nTSC}, \Theta}\left(\mathbf{k}+2 \mathbf{k}_{f}\right)\right]
\end{aligned}
$$

TABLE I. Symmetry breaking and collective modes. Here uTSC and nuTSC stand for unitary and nonunitary TSC, respectively, nTSC + AF corresponds to a mixed phase of non-unitary TSC and antiferromagnetism away from the $\mathrm{SO}(4)$ symmetric point.

\begin{tabular}{ccccc}
\hline \hline Phase & $\begin{array}{c}\text { Order } \\
\text { parameter }\end{array}$ & $\begin{array}{c}\text { Residual } \\
\text { symmetry }\end{array}$ & $\begin{array}{c}\text { Goldstone } \\
\text { (massless) modes }\end{array}$ & $\begin{array}{c}\text { Pseudo-Goldstone } \\
\text { (massive) modes }\end{array}$ \\
\hline AF & $\left\langle Q_{z z}\right\rangle$ & $\mathrm{U}(1) \times \mathrm{U}(1)\left(S_{z}, I_{z}\right)$ & $2\left(S_{x}, S_{y}\right)$ & $2\left(I_{x}, I_{y}\right)$ \\
uTSC & $\left\langle Q_{x x}\right\rangle$ & $\mathrm{U}(1)\left(S_{x}\right)$ & $3\left(S_{z}, S_{y}, I_{z}\right)$ & $1\left(I_{y}\right)$ \\
nuTSC & $\left\langle Q_{x x}\right\rangle=\left\langle Q_{y y}\right\rangle$ & $\mathrm{U}(1)\left(S_{z}+I_{z}\right)$ & $3\left(S_{z}, S_{y}, S_{z}-I_{z}\right)$ & $2\left(I_{y}, I_{x}\right)$ \\
nTSC+AF & $\left\langle Q_{x x}\right\rangle=\left\langle Q_{y y}\right\rangle,\left\langle Q_{z z}\right\rangle$ & $\mathrm{U}(1)\left(S_{z}+I_{z}\right)$ & $3\left(S_{z}, S_{y}, S_{z}-I_{z}\right)$ & $2\left(I_{y}, I_{x}\right)$ \\
unitary SO$(4)$ & $\left\langle Q_{x x}\right\rangle$ & $\mathrm{U}(1) \times \mathrm{U}(1)\left(I_{x}, S_{x}\right)$ & $4\left(S_{z}, S_{y}, I_{z}, I_{y}\right)$ & 0 \\
nonunitary $\mathrm{SO}(4)$ & $\left\langle Q_{x x}\right\rangle=\left\langle Q_{y y}\right\rangle=\left\langle Q_{z z}\right\rangle$ & $\mathrm{SO}(3)(\mathbf{I}+\mathbf{S})$ & $3(\mathbf{I}-\mathbf{S})$ & 0 \\
\hline \hline
\end{tabular}




$$
\begin{aligned}
& \left|\left\langle 0\left|\left[b_{\Theta_{y}}(\mathbf{k}), Q_{z \alpha}(\mathbf{k})\right]\right| 0\right\rangle\right|^{2} \delta\left(\omega-\omega_{\mathrm{nTSC}, \Theta}\right) \\
& \quad=\delta_{\alpha x} \frac{\psi^{2}}{1+\chi_{2}\left(J k^{2} / 2+4 \delta r\right)} \delta\left[\omega-\omega_{\mathrm{nTSC}, \Theta}\left(\mathbf{k}+2 \mathbf{k}_{f}\right)\right] .
\end{aligned}
$$

The phase fluctuation mode in the nonunitary TSC phase differs from its counterpart in the unitary case. Equations (57)-(59) for $\left\{Q_{x y}, Q_{y x}, S_{z}, I_{z}\right\}$ cannot be decoupled, giving a mode that involves both spin and isospin generators. Due to the residual symmetry generated by $S_{z}+I_{z}$, the mode with $Q_{x y}-Q_{y x}$, drops out of the spectrum. The remaining excitation follows the dispersion

$$
\omega_{\mathrm{nTSC}, \varphi}(\mathbf{k})=\psi \sqrt{J\left(\frac{1}{\chi_{1}}+\frac{1}{\chi_{2}}\right)} k .
$$

As in the unitary case, Coulomb interactions make this a massive mode, with a mass of the order of the plasma en- ergy. The creation operator of this mode involves the generators $I_{z}$ and $S_{z}$. Substitution into Eq. (62) gives vanishing neutron scattering intensity at $2 \mathbf{k}_{f}$.

\section{Mixed phase}

In the AF/nonunitary TSC mixed phase, the order parameters form an orthogonal triad $\psi_{1}=\psi \hat{x}, \psi_{2}=\psi \hat{y}, \mathbf{N}=N \hat{z}$, i.e., $\left\langle Q_{x x}\right\rangle=\left\langle Q_{y y}\right\rangle=\psi$, and $\left\langle Q_{z z}\right\rangle=N$. It is easy to verify that the phase fluctuation mode remains unchanged, its dispersion given by Eq. (75). However, other modes are complicated due to the fact that Eqs. (57)-(59) couple the coordinates $\left\{Q_{x z}, Q_{z x}, S_{y}, I_{y}\right\}$ and similarly $\left\{Q_{y z}, Q_{z y}, S_{x}, I_{x}\right\}$. Solution of the eigenvalue equations yields two collective modes for each of the above coordinate sets. One is a massive " $\Theta$ " mode and the other a gapless spin wave-like mode:

$$
\omega_{S, \Theta}=\sqrt{\left(\frac{2 \delta r \phi}{\chi_{2}}+\frac{J_{\mathbf{k}} \rho}{2 \chi_{t}}\right) \mp \sqrt{\left(\frac{2 \delta r \phi}{\chi_{2}}+\frac{J_{\mathbf{k}} \rho}{2 \chi_{t}}\right)^{2}-\frac{J_{\mathbf{k}}}{\chi_{1} \chi_{2}}\left(J_{\mathbf{k}} \phi^{2}+4 \delta r \phi \rho\right)},}
$$

where $\chi_{t}^{-1} \equiv \chi_{1}^{-1}+\chi_{2}^{-1}, J_{\mathbf{k}} \equiv J z / 2\left(1-\gamma_{\mathbf{k}}\right), \phi \equiv \psi^{2}-N^{2}$, and $\rho$ $\equiv \psi^{2}+N^{2}$. To calculate the spectrum in the mixed phase as a function of the tuning parameter $\delta r$, we find the values of the order parameters at a given $\delta r$ from the mean field theory of Eq. (55). Specifically we use the result $\psi^{2}-N^{2}=\delta r / \widetilde{u}_{2}$. Figure 11(a) gives an example of the dispersions obtained for a particular value of $\delta r$ within the mixed phase. The asymptotic form of the excitation energies at small wave vectors is given by:

$$
\begin{gathered}
\omega_{S} \sim \sqrt{\frac{J \rho}{2 \chi_{1}}}|\mathbf{k}| \\
\omega_{\Theta} \sim \sqrt{\frac{J \rho}{2 \chi_{2}} k^{2}+\frac{4 \delta r^{2}}{\widetilde{u}_{2} \chi_{2}} .}
\end{gathered}
$$

Figure 11, demonstrates that the exact dispersion Eq. (76) deviates from these asymptotic forms already at relatively small wave vectors. This is due to the strong mixing between spin and isospin modes. Due to this mixing, both spin waves and $\Theta$ modes carry some weight in the neutron scattering intensity [see Fig. 11(b)]. The scattering intensity associated with the spin wave mode vanishes in the vicinity of $\delta r=0$. On the other hand the intensity of the $\Theta$ modes becomes dramatically enhanced. Another unique feature of the phase with mixed nonunitary TSC and AF order is a linear with $|\delta r|$ softening of the $\Theta$-excitation gap. Compare this to the $\sqrt{\delta r}$ softening in the unitary TSC (see also Fig. 12).
The $\mathrm{SO}(4)$ symmetric point $\delta r=0$ needs special consideration. Here $N^{2}=\psi^{2}$ and the order parameter is invariant under the $\mathbf{S O}(3)$ group generated by $\mathbf{I}+\mathbf{S}$. This implies that there are only three Goldstone modes at this point. Indeed, a direct calculation at the $\mathrm{SO}(4)$ symmetric point gives three degenerate modes with dispersion:

$$
\omega_{\mathrm{nSO}(4)}(k)=\psi \sqrt{\frac{J}{2}\left(\frac{1}{\chi_{1}}+\frac{1}{\chi_{2}}\right)}|\mathbf{k}| .
$$

Note that the number of Goldstone modes at the $\mathrm{SO}(4)$ point is different in a unitary and nonunitary TSC. In the unitary case the spin and isospin $\mathrm{SO}(3)$ symmetries are broken separately with a residual $U(1) \times U(1)$ symmetry of the order parameter local gauge freedom associated with each. This leads to four Goldstone modes. In the nonunitary case, on the other hand, there is residual $\mathrm{SO}(3)$ symmetry of the order parameter, corresponding to $\mathbf{I}+\mathbf{S}$ rotations as discussed above. Consequently, this system has only three Goldstone modes.

The gapless spin waves and phase modes that we found away from the $\mathrm{SO}(4)$ symmetry are generic to systems that break spin $\mathrm{SO}(3)$ and charge $\mathrm{U}(1)$ symmetries. However, $\Theta$ excitations, which can be thought of as massive isospin waves, are not. Their presence shows the proximity of AF and TSC phases and their softening at the point $\delta r=0$ should provide a unique signature of the $\mathrm{SO}(4)$ symmetry of the system. 


\section{SO(4) SYMMETRY IN A STRONGLY ANISOTROPIC FERMI LIQUID}

Thus far in the analysis we have treated the case of weakly coupled Luttinger liquids, where we showed that $\mathrm{SO}(4)$ symmetry describes the phase diagram and collective modes of a system near the AF/TSC phase transition. However, there is a tendency away from Luttinger behavior as pressure is increased towards the superconducting state, as supported by the observation of field-induced SDW phases at high magnetic fields, ${ }^{99-104}$ by optical measurements, ${ }^{105}$ and by low temperature transport experiments. ${ }^{48}$ A review of the normal state of Bechgaard salts at low magnetic fields is given in Ref. 47. In this section, we will consider the effects of interchain hopping in the extreme case where it is large enough to destroy all remnants of Luttinger liquid physics, and make the system into a highly anisotropic Fermi liquid instead. We will see that even in this limit, despite the loss of nesting, an approximate $\mathrm{SO}(4)$ symmetry remains.

We begin by looking at the classical $\mathrm{SO}(4)$ symmetry of the GL free energy. In order to investigate this symmetry, it is sufficient to consider the quartic GL terms. This follows from the fact that our analysis of the phase diagram includes explicitly anisotropy in the quadratic terms, see Eq. (29), which is tuned to zero by pressure at the AF/TSC transition. As shown in Appendix B, a microscopic derivation of the GL parameters starting from a weakly interacting Fermi liquid leads to the following form for the quartic GL terms:

$$
\begin{aligned}
F_{4}= & A\left(2\left(|\boldsymbol{\Psi}|^{2}\right)^{2}-\left|\boldsymbol{\Psi}^{2}\right|^{2}\right)+B\left(\mathbf{N}^{2}\right)^{2} \\
& +2(C+D)|\boldsymbol{\Psi}|^{2} \mathbf{N}^{2}-4 D|\boldsymbol{\Psi} \cdot \mathbf{N}|^{2},
\end{aligned}
$$

where, for a perfectly nested Fermi surface, $A=B=C / 2=D$ $=7 \zeta(3) / 16 \pi^{2} v_{f} T^{2}$ satisfy the $\mathrm{SO}(4)$ symmetry conditions (31)

$$
\begin{gathered}
A=D, \\
B=D, \\
C / 2=D,
\end{gathered}
$$

and the sign of the coefficients corresponds to the unitary TSC case. In the presence of interchain coupling $t_{b}$, the single electron spectrum becomes

$$
\xi_{\mathbf{k}}=-2 t_{a} \cos k_{a}-2 t_{b} \cos k_{b}-\mu .
$$

Although Bechgaard salts are triclinic, and expression (80) applies to rectangular lattices only, it gives a good approximation to the low energy quasiparticle states of the system. Here, we take $t_{a}=250 \mathrm{meV}, t_{b}=20 \mathrm{meV}$, as estimated from plasma frequency measurements ${ }^{106}$ and band structure calculations. ${ }^{107}$ In addition, we take $\mu=\sqrt{2} t_{a}$, corresponding to a quarter-filled band and a nesting vector $\mathbf{Q}=\left(2 k_{f}, \pi\right)$ $\approx(\pi / 2, \pi)$. We note that, in the Fermi liquid description, dimerization only affects very high energy quasiparticles, and we exclude it from Eq. (80).

At first glance, interchain hopping seems to have a devastating effect on the $\mathrm{SO}(4)$ symmetry. The nesting vector $\mathbf{Q}$ no longer connects the right and left moving Fermi surfaces

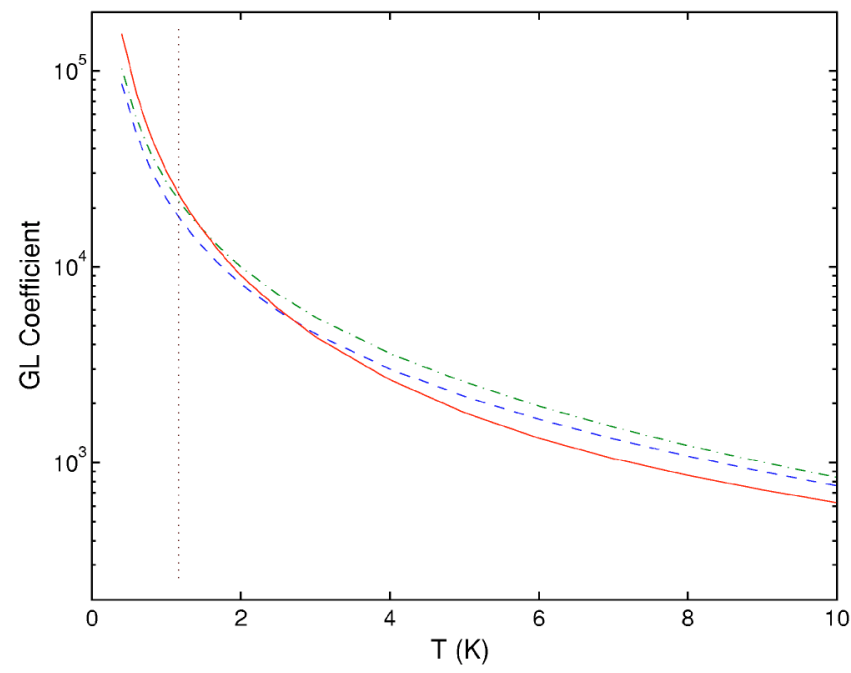

FIG. 13. Quartic Ginzburg-Landau coefficients (79) for a strongly anisotropic Fermi liquid, for the choice of parameters $t_{a}$ $=250 \mathrm{meV}, t_{b}=20 \mathrm{meV}$. The solid curve shows $\sqrt{A B}$, the dashed curve $D$, and the dash-dotted curve $(C+D) / 3$. Although the three curves do not coincide, as would be required by SO(4) symmetry, they trace similar trajectories all the way down to the critical temperature $T_{c}=1.2 \mathrm{~K}$ (vertical dotted line). At temperatures higher than shown, the three curves converge as nesting is restored.

exactly. Hence, while the coefficient $A$ is insensitive to $t_{b}$, the low temperature divergence in the coefficients $B, C$, and $D$ is preempted by the loss of nesting. Instead, these coefficients saturate at a temperature of the order of $t_{b}^{2} / t_{a} \approx 20 \mathrm{~K}$, changing the ratio $A / B$ from unity at high temperatures to about 10 at $T_{c}=1.2 \mathrm{~K}$. However, nesting strongly affects antiferromagnetism only, and not superconductivity. Hence, its effects on the GL parameters grow in proportion to the number of times that each GL parameter multiplies $\mathbf{N}$ in Eq. (79). Thus, most of the effect can be absorbed into the normalization of the field $\mathbf{N}$. While the fields $\mathbf{N}$ and $\boldsymbol{\Psi}$ cannot be normalized independently in the full GL free energy, as this would change the ratio of gradient terms $\frac{1}{2}\left[|\nabla \mathbf{\Psi}|^{2}+(\nabla \mathbf{N})^{2}\right]$, such scaling is allowed when considering the mean-field properties of the system. The conditions for $\mathrm{SO}(4)$ symmetry at mean-field level are then

$$
\begin{gathered}
\sqrt{A B}=D, \\
(C+D)=3 D .
\end{gathered}
$$

Thus, at the mean-field level, $\mathrm{SO}(4)$ symmetry is only broken weakly. This is illustrated in Fig. 13, where the left- and right-hand sides of the conditions Eq. (81) are evaluated explicitly. Despite the strong variation in the values of the different GL coefficients, the curves shown in Fig. 13 trace similar trajectories, indicating the approximate $\mathrm{SO}(4)$ symmetry. At $T_{c}=1.2 \mathrm{~K}$, we find $A=7.0 \times 10^{4}, B=7.3 \times 10^{3}, C$ $=3.1 \times 10^{4}$, and $D=2.1 \times 10^{4}$, leading to $\sqrt{A B} / D=1.06$ and $(C+D) /(3 D)=0.82(\mathbf{N}$ was rescaled by a factor of 1.76$)$. Thus, the conditions Eq. (81) deviate from exact $\mathrm{SO}(4)$ symmetry by less than $20 \%$ at $T_{c}=1.2 \mathrm{~K}$. 
While classical SO(4) symmetry is no longer exact, the phase diagram derived in previous sections does not change in important ways. At the mean-field level, the $20 \%$ variation from the $\mathrm{SO}(4)$ conditions $\mathrm{Eq}$. (81) can open a very narrow mixed phase between the TSC and AF phases. Hence, as pressure is varied from the AF phase to the TSC phase, there will no longer be a discontinuity in the order parameters or in the density. Instead, these quantities will show a smooth but very rapid variation as pressure transverses the mixed phase. Therefore, due to the narrowness of the mixed phase, the system will be very sensitive to disorder. For realistic systems, which have impurities and crystal defects, the mixed phase will segregate into inhomogeneous regions of $\mathrm{AF}$ and TSC, just as found in the coexistence phase in the strictly first order case. However, unlike the case of a first order transition, the inhomogeneous behavior will be apparent even when the phase diagram is tuned by experimental variables that are intrinsic. We note that the narrowness of the mixed phase, and the corresponding sensitivity to disorder, is a direct consequence of the proximity of the system to $\mathrm{SO}(4)$ symmetry.

We now consider the fluctuation-induced first order transition between the AF and normal phases near the $\mathrm{SO}(4)$ symmetric point. We note that in order to alter the topology of the phase diagram, the bare GL parameters must differ enough from the $\mathrm{SO}(4)$ symmetric values to divert the $\mathrm{RG}$ flow near a new critical point. While symmetry can play an important role in an RG flow, it is difficult to conceive of a situation where reduction of symmetry would lead to softening of the first order transitions into second order. Thus, we expect the first order transition between $\mathrm{AF}$ and normal phases discussed earlier to still be present. Finally, we note that the case we consider in this section is extreme, in that we look study the system as a weakly interacting, strongly anisotropic Fermi liquid. This probably gives a strong overestimate of the magnitude of the breaking of classical $\mathrm{SO}(4)$ symmetry in real systems, which are likely to lie between the Fermi liquid limit and the weakly coupled Luttinger liquid limit, where $\mathrm{SO}(4)$ is a good symmetry.

Before concluding this section, we briefly discuss the effects of interchain coupling on the quantum $\mathrm{SO}(4)$ symmetry. One can look for this symmetry by verifying the existence of the $\Theta$ resonance in a strongly anisotropic Fermi liquid formalism. Inside the TSC phase, this can be done using RPA type calculations, which include the AF particle-hole and $\Theta$ resonance particle-particle channels (see, e.g., Ref. 108). Results of these calculations will be reported elsewhere. The main effect of interchain hopping is to fix the transverse components of the nesting vector to $\mathbf{Q}=\left(2 k_{f}, \pi, \pi\right)$. Thus, the $\Theta$ resonance for quasi-one-dimensional systems is a collective mode, whose quantum numbers are spin zero, charge two, and wave vector $\mathbf{Q}$. The presence of interchain coupling also introduces broadening of the $\Theta$ mode, and prevents it from softening all the way down to zero energy at the $\mathrm{AF} /$ TSC phase transition. Instead, we expect the minimum energy of the excitation to be of the order of $t_{b}^{2} / t_{a}=16 \mathrm{meV}$.

\section{EXPERIMENTAL SIGNATURES OF THE SO(4) SYMMETRY}

The interplay of $\mathrm{AF}$ and $\mathrm{SC}$ in the organic material $(\mathrm{TMTSF})_{2} \mathrm{PF}_{6}$ has been a subject of active investigation. ${ }^{7-9}$
There is strong experimental evidence supporting that superconducting order is spin triplet, as discussed in Sec. I. In addition, (TMTSF) ${ }_{2} \mathrm{PF}_{6}$ has a quasi-one-dimensional structure, as the anisotropy of electron tunneling along the chains (a), in the planes (b), and perpendicular to the planes (c) is of the order of $t_{a}: t_{b}: t_{c}=250: 25: 1$. Hence, $(\mathrm{TMTSF})_{2} \mathrm{PF}_{6}$ is a good candidate for comparison with the theoretical model discussed in this paper. Following the discussion in Sec. III and Appendix B we expect the triplet order parameter in this material to be unitary. The phase diagram for this case was obtained in Sec. V. In Ref. 55 we compare this to the experimental phase diagram of (TMTSF) ${ }_{2} \mathrm{PF}_{6} \cdot{ }^{10,11}$ One consequence of having enhanced symmetry at a phase transition is the suppression of the critical temperature due to fluctuations of one order parameter into the other. This may contribute to the drastic drop in $T_{\mathrm{AF}}$ as pressure is increased near the $\mathrm{AF} /$ TSC phase boundary in Bechgaard salts. ${ }^{10}$

The first order transition between AF and TSC phases near the critical point, Fig. 6, leads to a regime of frustrated phase separation, with domains of one phase inside the other. The volume fractions of each phase are governed by the Maxwell construction, while the size of individual domains is determined by the competition between short-range and long-range parts of the Coulomb interaction. ${ }^{109}$ If the domains are distributed randomly, the total resistance of the system may be found using an effective medium approximation. This implies, for example, that the system is superconducting when the TSC phase is beyond the percolating threshold. On the other hand, it is possible that the TSC domains are not distributed uniformly in the system, and are more favorable on the surface of the sample. In this case, the TSC regions can "short-circuit" the system even before they reach the percolation condition for the bulk. Transport properties consistent with this scenario of an inhomogeneous system have been reported in Ref. 10.

An interesting direction for exploring competition between AF and TSC phases is to use magnetic field experiments in the superconducting state near the AF/TSC phase boundary. Magnetic field produces orbital currents that strongly suppress electron pairing and leads to a formation of an Abrikosov vortex lattice. Suppression of the AF order by Zeeman effect is much smaller. Thus, we expect magnetic fluctuations to become strongly enhanced in the mixed state. ${ }^{30,62,110-112}$ Since the critical field along the $c$ axis is $H_{c 2}^{c}=100 \mathrm{mT},{ }^{18}$ an applied magnetic field along the $c$ axis on the order of a few mT can have a strong effect, see Fig. 14. This is in contrast with effects such as field induced SDWs and reentrant superconductivity, which require fields of at least $5 \mathrm{~T}$ for their observation. ${ }^{1,99-104}$ For pressures close to the AF/TSC phase boundary and for slightly larger magnetic fields there may also be a quantum phase transition in which long range $\mathrm{AF}$ order develops inside the vortex phase. We note that strong sensitivity of $1 / T_{1}$ to magnetic fields in the superconducting state of (TMTSF) ${ }_{2} \mathrm{PF}_{6}$ have been reported in Ref. 20. Here, increasing the magnetic field along the $b$ axis from 12.8 to $232 \mathrm{mT}$ results in a large increase of $1 / T_{1}$, consistent with the enhancement of antiferromagnetism that we propose. Earlier specific heat measurements in Ref. 113 already showed that when the superconducting 


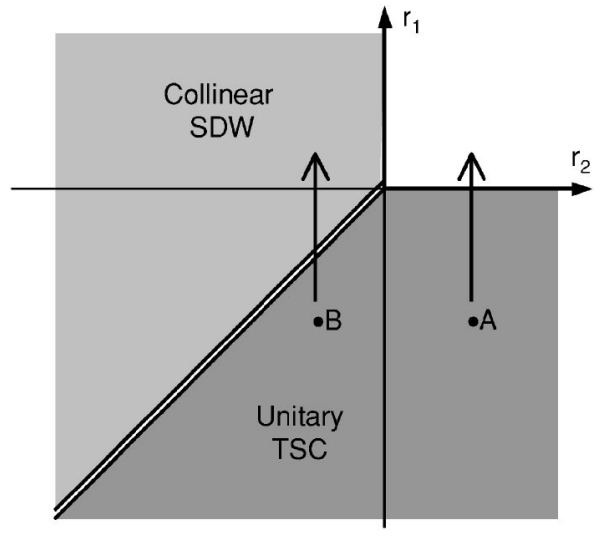

FIG. 14. The effect of a magnetic field on the superconducting state. For points (A) far from the AF/TSC boundary, the magnetic field destroys superconductivity leading to a normal state. For points (B) close to the boundary, a magnetic phase is stabilized instead. The double line denotes a first order transition, which expands into a AF/TSC coexistence region in the experimental phase diagram. Here we focus on the unitary case, but similar effects can be seen for the nonunitary case near the AF/TSC mixed phase. Fields of the order of $100 \mathrm{mT}$ are sufficient for a significant enhancement in antiferromagnetism to be observed. This is in contrast with field-induced SDW phases, which require fields in excess of $5 \mathrm{~T}$.

order in (TMTSF) $)_{2} \mathrm{ClO}_{4}$ is suppressed by a magnetic field, the system goes into a semimetallic state with a suppressed quasiparticle density of states. This is consistent with developing AF order, thus opening a gap in the quasiparticle spectrum. It may be interesting to study further the enhancement of magnetic order in the mixed state with neutron scattering, ${ }^{114-117} \mathrm{NMR},{ }^{118-120}$ and $\mu \mathrm{SR}^{121}$ experiments.

In Ref. 55 (see also Sec. VII of this paper) we discuss that direct observation of the $\Theta$ mode in the superconducting phase should be possible through neutron scattering. The most important feature of the $\Theta$ resonance, which identifies it as a generator of the $\mathrm{SO}(4)$ symmetry, is the pressure dependence of the resonance energy inside the TSC phase. When the pressure is reduced and the system is brought toward the phase boundary with the AF phase, we predict the energy of the $\Theta$ resonance to be dramatically decreased. Mode softening is not expected generically at first order phase transitions and provides a unique signature of the $\mathrm{SO}(4)$ quantum symmetry. We note that due to interchain hopping, the center of mass momentum of the $\Theta$ excitation in quasi-one-dimensional systems is $\left(2 k_{f}, \pi, \pi\right)$.

Another approach to detect the $\Theta$ excitation involves tunneling experiments with the $\mathrm{SSC} /(\mathrm{TMTSF}){ }_{2} \mathrm{ClO}_{4}$ junction shown in Fig. 15 (analogous experiments in the context of $\pi$ excitations in the high $T_{c}$ cuprates are discussed in Ref. 122). A singlet superconductor provides a reservoir of Cooper pairs that can couple to $\Theta$ pairs in (TMTSF) ${ }_{2} \mathrm{ClO}_{4}$. One needs to overcome, however, the momentum mismatch between the two types of pairs. A possible approach is to use an intermediate layer of the quasi- $1 d$ material (TMTTF) ${ }_{2} \mathrm{PF}_{6}$. This salt is quarter filled and displays spin-Peierls (SP) order. The modulations of the SP order thus have a periodicity of four TMTTF sites, matching the $\left(2 k_{f}, \pi, \pi\right)$ wave vector of

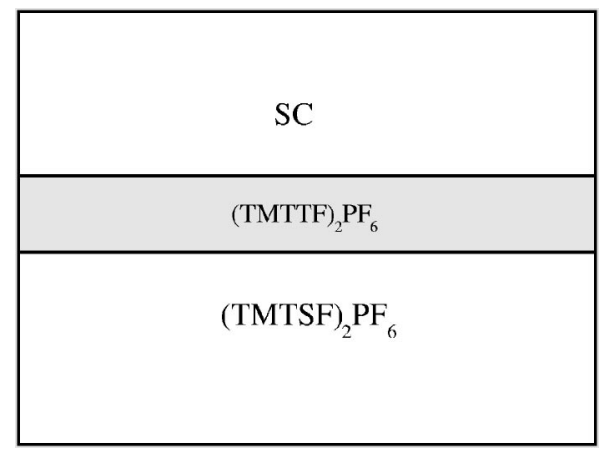

FIG. 15. Tunneling experiment for detecting the $\Theta$ excitation in (TMTSF) ${ }_{2} \mathrm{ClO}_{4}$ material. A singlet superconducting material with a higher transition temperature than (TMTSF) ${ }_{2} \mathrm{ClO}_{6}$ provides a reservoir of Cooper pairs that can couple resonantly to $\Theta$ pairs. Momentum mismatch between the Cooper pairs in $\mathrm{SC}$ and $\Theta$ pairs in (TMTSF) ${ }_{2} \mathrm{ClO}_{4}$ is compensated by scattering of electrons in a layer of the SP material (TMTTF) ${ }_{2} \mathrm{PF}_{6}$.

(TMTSF) ${ }_{2} \mathrm{ClO}_{4}$. The small mismatch between the two wave vectors, due to differences in the lattice constant in these compounds, can be compensated by a parallel magnetic field. ${ }^{123}$ We expect peaks in the current-voltage characteristics of the junction when the voltage bias compensates the energy difference between Cooper and $\Theta$ pairs

$$
2 e V=\omega_{\Theta} .
$$

Peaks in $I V$ should be present even above the superconducting transition temperature of (TMTSF) ${ }_{2} \mathrm{ClO}_{4}$ and only require the other material to be superconducting. The choice of (TMTSF) ${ }_{2} \mathrm{ClO}_{4}$ is made as this material is likely to be close to the AF/TSC transition at ambient pressure. ${ }^{124}$ This eliminates the need for pressure cells, which would make the experiments much more difficult.

\section{SUMMARY}

The primary purpose of this paper has been to discuss the competition of antiferromagnetism and triplet superconductivity in quasi-one-dimensional systems, such as Bechgaard salts (TMTSF) ${ }_{2} \mathrm{X}$. The point of departure of our work is the existence of enhanced symmetry, that unifies the two order parameters, in one-dimensional systems of interacting electrons. Analysis of the Luttinger liquid model presented in Sec. II showed that the usual charge and spin $\mathrm{U}(1) \times \mathrm{SO}(3)$ symmetry is enhanced to a higher $\mathrm{SO}(3) \times \mathrm{SO}(4)$ symmetry on the transition line between the two phases for incommensurate band filling. For half-filled systems and weak umklapp scattering, the enhanced symmetry group becomes $\mathrm{SO}(4)$. Weak coupling between chains, that enables true long range order, is expected to perturb the $\mathrm{SO}(4)$ symmetry only slightly.

In Secs. III-VI we studied the finite temperature phase diagram for systems with $\mathrm{SO}(4)$ symmetry. For the unitary case, a mean field analysis shows that $\mathrm{SO}(4)$ symmetry requires a direct first order transition between TSC and AF phases. In addition, fluctuations of the order parameters turn a portion of the boundary between AF and normal phases 
into a first order transition and also lead to a weakly first order transition between TSC and normal phases. For the nonunitary case, $\mathrm{SO}(4)$ symmetry requires two second order transitions between TSC and AF phases. We find that the system is close to having a $\mathrm{SO}(4)$ symmetric tetracritical point, but thermal fluctuations stretch this point into a short line of direct first order transitions from the normal state to the mixed state. Our results have direct implications for quasi-one-dimensional organic superconductors from the $(\mathrm{TMTSF})_{2} \mathrm{X}$ family, which are likely to be unitary triplet superconductors. For example, first order transitions between the AF and the TSC phases, and between the Normal and the $\mathrm{AF}$ phases explain the AF/TSC and the AF/Normal coexistence regions found in the phase diagram of $(\mathrm{TMTSF})_{2} \mathrm{PF}_{6}$.

In Sec. VII we analyze collective excitations in various phases and demonstrate that $\mathrm{SO}(4)$ leads to the existence of a new collective mode, the $\Theta$ excitation, which describes rotations between the AF and the TSC phases. In Sec. IX we study possible experimental tests of the $\mathrm{SO}(4)$ symmetry. We propose that the $\Theta$ excitation should be observed as a sharp resonance in spin polarized inelastic neutron scattering experiments in the superconducting phase. We predict that the energy of the peak decreases toward the first order phase transition to AF order. Such softening of modes is not expected in general near a first order transition and would be a unique signature of the enhanced symmetry at the transition point.

\section{ACKNOWLEDGMENTS}

The authors thank S. Brown, P. Chaikin, M. Dressel, B.I. Halperin, C. Kilic, S. Sachdev, A. Turner, D.-W. Wang, and S.C. Zhang for useful discussions. This work was supported by Harvard NSEC.

\section{APPENDIX A: DERIVATION OF SO(3) XSO(4) SYMMETRY IN LUTTINGER LIQUIDS}

In this Appendix we demonstrate that along the line $g_{1}$ $=2 g_{2}$, the Luttinger liquid Hamiltonian (1) has an exact $\mathrm{SO}(3)_{\text {spin }} \times \mathrm{SO}(4)_{\text {isospin }}$ symmetry. For this, we use bosonization to write the $\Theta_{ \pm}$operators $(6)$ as $(r= \pm)$ :

$$
\Theta_{r}^{\dagger}=r \int d x \frac{\eta_{r \uparrow} \eta_{r \downarrow}}{2 \pi \alpha} e^{-r A(x)},
$$

where $A(x)=i \sqrt{2}\left[\phi_{\rho}(x)+\theta_{\rho}(x)\right]$ and $\theta_{\rho}=\pi \int^{x} d x^{\prime} \Pi_{\rho}\left(x^{\prime}\right)$. Note that $\Theta_{r}^{\dagger}$ are independent of the spin fields $\phi_{\sigma}$ and $\theta_{\sigma}$. Hence, the spin sector of the bosonized Hamiltonian commutes trivially with $\Theta_{r}^{\dagger}$, and we need only keep track of the charge sector

$$
\mathcal{H}_{\rho}=\int d x\left(\frac{\pi u_{\rho} K_{\rho}}{2} \Pi_{\rho}^{2}+\frac{u_{\rho}}{2 \pi K_{\rho}}\left(\partial_{x} \phi_{\rho}\right)^{2}\right) .
$$

Whenever $g_{1}=2 g_{2}$, corresponding to $K_{\rho}=1$, the commutator $\left[\mathcal{H}_{\rho}, A\right]$ takes on a simple form:

$$
\left[\mathcal{H}_{\rho}, A(x)\right]=\sqrt{2} u_{\rho}\left[\partial_{x} \phi_{\rho}(x)+\pi \Pi_{\rho}(x)\right]=-i u_{\rho} \partial_{x} A(x)
$$

so that commuting $\mathcal{H}_{\rho}$ with an arbitrary function of $A(x)$ is equivalent to taking the derivative with respect to $x$. For example,

$$
\begin{aligned}
{\left[\mathcal{H}_{\rho}, e^{A}\right] } & =\sum_{n} \frac{1}{n !}\left[\mathcal{H}_{\rho}, A^{n}\right] \\
& =\left[\mathcal{H}_{\rho}, A\right]+\frac{1}{2}\left(A\left[\mathcal{H}_{\rho}, A\right]+\left[\mathcal{H}_{\rho}, A\right] A(x)\right)+\cdots \\
& =-i u_{\rho}\left(\partial_{x} A+\frac{1}{2}\left(A \partial_{x} A+\partial_{x} A A\right)\right)+\cdots \\
& =-i u_{\rho} \partial_{x} e^{A}
\end{aligned}
$$

Hence,

$$
\left[\mathcal{H}_{\rho}, \int d x e^{A(x)}\right]=-i u_{\rho} \int d x \partial_{x} e^{A(x)}=-i u_{\rho}\left[e^{A(L)}-e^{A(0)}\right],
$$

which vanishes if periodic boundary conditions are imposed on $\phi(x)$ and $\theta(x)$. Thus, for $K_{\rho}=1$,

$$
\left[\mathcal{H}, \Theta_{ \pm}^{\dagger}\right]=0,
$$

and the Luttinger liquid has full $\mathrm{SO}(3)_{\text {spin }} \times \mathrm{SO}(4)_{\text {isospin }}$ symmetry, generated by $\Theta_{ \pm}$, the total spin operators $S_{\alpha}$, and the charge of left and right movers,

$$
Q_{ \pm}=\sum_{k s}\left(a_{ \pm, k s}^{\dagger} a_{ \pm, k s}-\frac{1}{2}\right) .
$$

The enlarged symmetry relies on the independent conservation of total number of right and left movers. This is not a good conservation law, for instance, in the presence of impurity scattering, dimerization, or umklapp. For general $K_{\rho}$, we find

$\left[\mathcal{H}, \Theta_{ \pm}^{\dagger}\right]=\frac{K_{\rho}^{2}-1}{2 K_{\rho}} \int d x \sqrt{2} u_{\rho}\left[\partial_{x} \phi_{\rho}(x)-\pi \Pi_{\rho}(x)\right] \frac{\eta_{ \pm \uparrow} \eta_{ \pm \downarrow}}{2 \pi \alpha} e^{\mp A(x)}$.

We would like to thank Daw-Wei Wang for helpful discussions on results presented in this section.

\section{APPENDIX B: PARAMETERS OF THE GINZBURG- LANDAU FREE ENERGY FOR WEAK INTERACTIONS}

To extract parameters of the GL free energy we consider a mean-field Hamiltonian

$$
\begin{aligned}
\mathcal{H}= & \sum_{k s}\left(\epsilon_{k}-\mu\right) a_{k s}^{\dagger} a_{k s}+\boldsymbol{\Psi} \cdot \sum_{k} \mathrm{w}_{k} a_{k s}^{\dagger} a_{-k s^{\prime}}^{\dagger}\left(\boldsymbol{\sigma} \sigma_{2}\right)_{s s^{\prime}} \\
& +\boldsymbol{\Psi}^{*} \cdot \sum_{k} \mathrm{w}_{k} a_{-k s^{\prime}} a_{k s}\left(\sigma_{2} \boldsymbol{\sigma}\right)_{s^{\prime} s}+\boldsymbol{\Phi} \cdot \sum_{k} a_{k-k_{f} s}^{\dagger} a_{k+k_{f} s^{\prime}} \boldsymbol{\sigma}_{s s^{\prime}} \\
& +\boldsymbol{\Phi} * \cdot \sum_{k} a_{k+k_{f} s^{\dagger}}^{\dagger} a_{k-k_{f} s^{\prime}} \boldsymbol{\sigma}_{s s^{\prime}}
\end{aligned}
$$




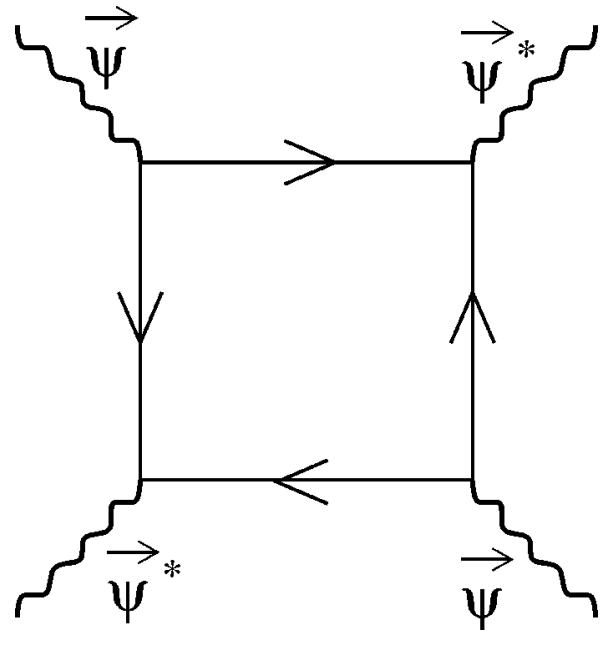

FIG. 16. Diagram for the coefficient $A$ in the GL free energy (B2).

where $\mathrm{w}_{k}=|k| / k$ gives the sign of $k$. Integrating out the fermions yields an effective action for the order parameter fields. We obtain the fourth order terms:

$$
\begin{aligned}
F_{4}= & A\left(2\left(|\boldsymbol{\Psi}|^{2}\right)^{2}-\left|\boldsymbol{\Psi}^{2}\right|^{2}\right)+B\left(2\left(|\boldsymbol{\Phi}|^{2}\right)^{2}-\left|\boldsymbol{\Phi}^{2}\right|^{2}\right)+2 C|\boldsymbol{\Psi}|^{2}|\boldsymbol{\Phi}|^{2} \\
& +2 D\left(|\boldsymbol{\Psi}|^{2}|\boldsymbol{\Phi}|^{2}-|\boldsymbol{\Phi} \cdot \boldsymbol{\Psi}|^{2}-|\boldsymbol{\Phi} * \cdot \boldsymbol{\Psi}|^{2}\right)
\end{aligned}
$$

where

$$
\begin{aligned}
A= & \frac{1}{2 \beta} \sum_{\omega_{n}} \int \frac{d k}{2 \pi} G^{2}\left(-k,-\omega_{n}\right) G^{2}\left(k, \omega_{n}\right) \\
B= & \frac{1}{\beta} \sum_{\omega_{n}} \int \frac{d k}{2 \pi} G^{2}\left(k, \omega_{n}\right) G\left(k+2 k_{f}, \omega_{n}\right)\left[G\left(k+2 k_{f}, \omega_{n}\right)\right. \\
+ & \left.2 G\left(k-2 k_{f}, \omega_{n}\right)\right] \\
C= & \frac{1}{\beta} \sum_{\omega_{n}} \int \frac{d k}{2 \pi} G^{2}\left(k, \omega_{n}\right) G\left(-k,-\omega_{n}\right)\left[G\left(k+2 k_{f}, \omega_{n}\right)\right. \\
+ & \left.G\left(k-2 k_{f}, \omega_{n}\right)\right] \\
D= & \frac{1}{\beta} \sum_{\omega_{n}} \int \frac{d k}{2 \pi} G\left(k, \omega_{n}\right) G\left(-k,-\omega_{n}\right) G\left(k+2 k_{f}, \omega_{n}\right) \\
& \times G\left(-k-2 k_{f},-\omega_{n}\right) \mathrm{w}_{-k} \mathrm{w}_{k+2 k_{f}} .
\end{aligned}
$$

For instance, the diagram giving the coefficient $A$ is shown in Fig. 16.

For the Luttinger liquid type model with linearized spectrum around $k= \pm k_{f}$ we obtain

$$
A=B=C / 2=D .
$$

The relationship among coefficients [Eq. (B4)] implies that the effective GL free energy [Eq. (B2)] is $\mathrm{SO}(3)_{\text {spin }}$ $\times \mathrm{SO}(4)_{\text {isospin }}$ symmetric, as expected from the discussion in Sec. II. $F$ can thus be parameterized in the form (22), with $\tilde{u}_{1}=3 A$ and $\tilde{u}_{2}=-2 A$. In the clean limit,
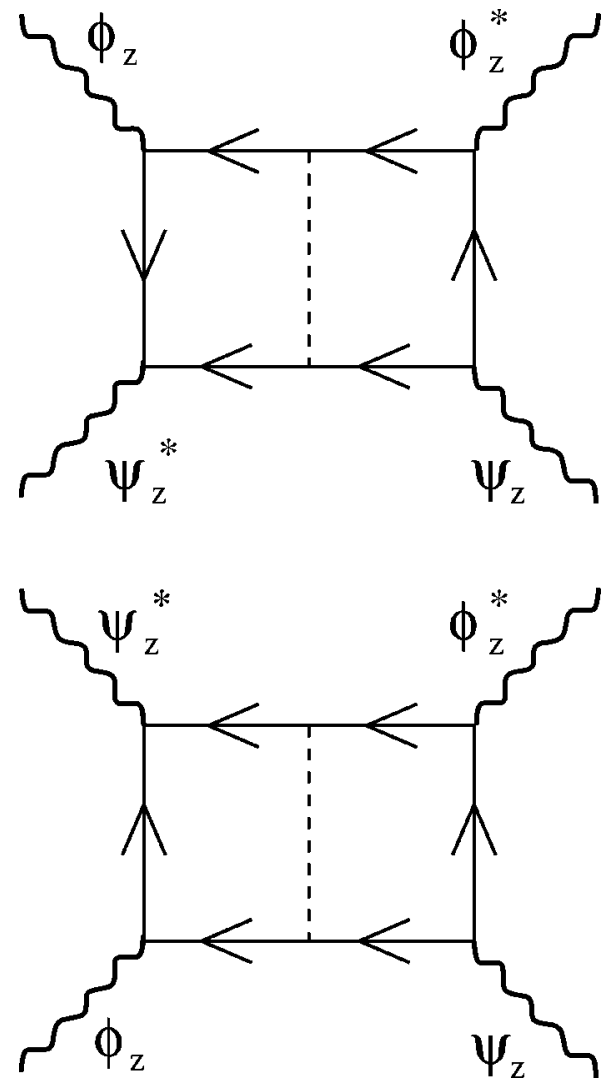

FIG. 17. Corrections to coefficient of $\left|\Phi_{z}\right|^{2}\left|\Psi_{z}\right|^{2}$ term in GL free energy, to linear order in umklapp scattering (dashed line). The two diagrams add up to zero. Inspection of all such diagrams shows that the quartic coefficients of the GL free energy are not modified to linear order.

$$
A=\frac{7 \zeta(3)}{16 \pi^{3} v_{f} T^{2}},
$$

where $\zeta(3)=1.202$, etc., and $v_{f}$ is the Fermi velocity.

Note that, as was pointed out in Sec. III B, to linear order in $g_{3}$, umklapp does not affect the quartic coefficients of the free energy. For instance, the diagrams in Fig. 17 could contribute to the coefficient of the term $\left|\Phi_{z}\right|^{2}\left|\Psi_{z}\right|^{2}$. However, although they do not vanish individually, the two add up to zero. This is consistent with the Feynman-Hellman theorem which requires that the only corrections to the free energy to linear order in $g_{3}$ be given by the expectation value of the perturbation (14).

\section{APPENDIX C: NONUNITARY TRIPLET SUPERCONDUCTIVITY AND ANTIFERROMAGNETISM: EXPANSION FROM THE LOWER CRITICAL DIMENSION}

Here we outline some of the methods used in the RG calculation of the nonlinear model (48) in $d=2+\epsilon$ dimensions. The RG flow equations of a general nonlinear model can be computed using the formalism of Friedan. ${ }^{75}$ The fields in such models must satisfy constrains which force them to lie on some target space manifold $\mathcal{M}$. For instance, the usual 
nonlinear $\sigma$ model deals with a single $N$-component vector with a constrained fixed length, and $\mathcal{M}$ in this case is the $N-1$ dimensional sphere describing the locus of possible values of such vector. A local set of coordinates $\phi_{i}$ can be introduced on a patch of $\mathcal{M}$, in terms of which the free energy becomes

$$
F=\int d x g_{i j}(\phi(x)) \partial_{\mu} \phi^{i}(x) \partial_{\mu} \phi^{j}(x) .
$$

Unlike the original fields used to define the model, the fields $\phi_{i}(x)$ are unconstrained; all information regarding the original constrains is contained in the metric $g_{i j}$. The metric also contains the coupling constants of the system. In Friedan's formalism, the RG flow is thought of as a gradual deformation of the manifold as the short length degrees of freedom are integrated out. The RG equations can be written in a covariant way; to one loop order,

$$
\frac{\partial g_{i j}}{\partial l}=\epsilon g_{i j}-R_{i j},
$$

where $\epsilon=d-2$ and $R_{i j}$ is the Ricci tensor, which is determined uniquely by the metric.

In practice, whenever the manifold is a homogeneous space $G / H$, as in our case, it is simplest to work directly in the tangent space of the manifold, see Ref. 76 for a detailed discussion. In terms of the metric on the tangent space, $\eta_{a b}$, the $\mathrm{RG}$ equations become

$$
\frac{\partial \eta_{a b}}{\partial l}=\epsilon \eta_{a b}-R_{a b},
$$

where the Ricci tensor in the tangent space is given by

$$
R_{a b}=\sum_{I c} f_{a c}{ }^{I} f_{I b}{ }^{c}+\sum_{c d} \frac{\eta_{a a}^{2}-\left(\eta_{c c}-\eta_{d d}\right)^{2}}{4 \eta_{c c} \eta_{d d}} f_{a c}{ }^{d} f_{b c}{ }^{d}
$$

in terms of the structure factor constants of the group $G$

$$
\begin{gathered}
{\left[T_{a}, T_{b}\right]=f_{a b}{ }^{c} T_{c}+f_{a b}{ }^{I} T_{I}} \\
{\left[T_{I}, T_{b}\right]=f_{I b}{ }^{c} T_{c} .}
\end{gathered}
$$

Generators labeled by upper case indices are elements of Lie $H$, while lower case indices denote generators in Lie $G$-Lie $H$. In applying expression (C4), we assume that the generators have been chosen so that the structure factor constants (C5) are antisymmetric with respect to exchange of any two indices; such a choice is always possible. Equation (C4) is written in a noncovariant way to make the dependence on the coupling constants $\eta_{a b}$ explicit, and it shows the advantage of working in tangent space: the Ricci tensor is given directly in terms of the Lie algebra of $G$.

We briefly digress to discuss the Lie algebra of the group $\mathrm{SO}(N)$, which has $N(N-1) / 2$ generators corresponding to infinitesimal rotations in the planes $\left\langle m, m^{\prime}\right\rangle$, where the indices $m \neq m^{\prime}$ run through the $N$ independent axes. For instance, $\mathrm{SO}(3)$ has three generators, $T_{x}=\langle\hat{y}, \hat{z}\rangle, T_{y}=\langle\hat{z}, \hat{x}\rangle$, and $T_{z}$ $=\langle\hat{x}, \hat{y}\rangle$. Keeping in mind that $\left\langle m, m^{\prime}\right\rangle=-\left\langle m^{\prime}, m\right\rangle$ ("a clockwise rotation in the $x-y$ plane is a counterclockwise rotation in the $y-x$ plane"), we introduce a graphical representation: if we draw $N$ points on a sheet of paper, an arbitrary generator $\left\langle m, m^{\prime}\right\rangle$ can be represented by an arrow going from point $m$ to point $m^{\prime}$. The structure factor constants of the Lie algebra

$$
\begin{aligned}
{\left[\left\langle m, m^{\prime}\right\rangle,\left\langle n, n^{\prime}\right\rangle\right]=} & \delta_{m^{\prime} n}\left\langle m, n^{\prime}\right\rangle-\delta_{m^{\prime} n^{\prime}}\langle m, n\rangle-\delta_{m n}\left\langle m^{\prime}, n^{\prime}\right\rangle \\
& +\delta_{m n^{\prime}}\left\langle m^{\prime}, n\right\rangle
\end{aligned}
$$

can be written as a "generalized $\epsilon$ tensor,"

$$
\left[\left\langle m, m^{\prime}\right\rangle,\left\langle n, n^{\prime}\right\rangle\right]=\epsilon_{\left\langle m, m^{\prime}\right\rangle\left\langle n, n^{\prime}\right\rangle}\left\langle p, p^{\prime}\right\rangle\left\langle p, p^{\prime}\right\rangle,
$$

which has a simple interpretation in terms of the arrows described above: $\epsilon$ vanishes unless $\left\langle m, m^{\prime}\right\rangle,\left\langle n, n^{\prime}\right\rangle$, and $\left\langle p, p^{\prime}\right\rangle$ are the edges of a closed triangle. If they do form a closed triangle, count the number of times that the directions of the arrows must be flipped to turn it into an oriented triangle, i.e., one satisfying $m^{\prime}=n, n^{\prime}=p$, and $p^{\prime}=m$. If the number of flips is even, then $\epsilon=1$; otherwise $\epsilon=-1$. With this in mind, inspection of Eq. (C4) shows that, for groups $G$ based on $\mathrm{SO}(N)$, where $f_{a b}{ }^{c} \propto \epsilon_{a b}{ }^{c}$, the calculation of the Ricci tensor reduces almost entirely to the counting of triangles.

Armed with these tools, consider the nonlinear model Eq. (48),

$$
\begin{aligned}
F= & \int d^{d} x\left(\frac{1}{2 g_{1}}\left(\nabla \mathbf{e}_{1}\right)^{2}+\frac{1}{2 g_{2}}\left[\left(\nabla \mathbf{e}_{2}\right)^{2}+\left(\nabla \mathbf{e}_{3}\right)^{2}\right]\right. \\
& \left.+\frac{1}{g_{3}}\left[\left(\mathbf{e}_{1} \cdot \nabla \mathbf{e}_{2}\right)^{2}+\left(\mathbf{e}_{1} \cdot \nabla \mathbf{e}_{3}\right)^{2}\right]+\frac{1}{g_{4}}\left(\mathbf{e}_{2} \cdot \nabla \mathbf{e}_{3}\right)^{2}\right) .
\end{aligned}
$$

Model (C7) has the symmetry $\mathrm{SO}(N)$ of rotations of the $\mathrm{N}$-component vectors, and the symmetry $\mathrm{SO}(2)$ of internal rotations between $\mathbf{e}_{2}$ and $\mathbf{e}_{3}$. Hence, the symmetry group of Eq. (C7) is $G=\mathrm{SO}(N) \times \mathrm{SO}(2)$. The order parameter is a triad of mutually orthogonal vectors, $\Phi=\left(\mathbf{e}_{1} \mathbf{e}_{2} \mathbf{e}_{3}\right)$, and the ordered phase has residual symmetry $H=\mathrm{SO}(N-3)$ $\times \mathrm{SO}(2)_{\text {diag. }}$. The generators of $H$ leave the triad $\Phi$ invariant, whereas the generators in Lie $G$-Lie $H$ rotate the triad and are in one-to-one correspondence with the spin waves of the system.

We identify four types of spin waves, corresponding to the following classes of generators: $T_{a_{1}}$, which leave $\mathbf{e}_{\{2,3\}}$ untouched but rotate $\mathbf{e}_{1}$ into one of the remaining $N-3$ directions; $T_{a_{2}}$, which leave $\mathbf{e}_{1}$ untouched, but rotate either $\mathbf{e}_{2}$ or $\mathbf{e}_{3}$ into one of the remaining $N-3$ directions; $T_{a_{3}}$, of rotations in either the $\mathbf{e}_{1}, \mathbf{e}_{2}$ plane or the $\mathbf{e}_{1}, \mathbf{e}_{3}$ plane; and $T_{a_{4}}$, composed of the single generator of rotations in the $\mathbf{e}_{2}, \mathbf{e}_{3}$ plane. Each class furnishes an independent irreducible representation under the action of the group $H$, leading to four different spin wave velocities, and to four different coupling constants, $\eta_{1} \ldots \eta_{4}$,

$$
\begin{aligned}
\eta_{b c}= & \sum_{a_{1}} \eta_{1} \delta_{b a_{1}} \delta_{c a_{1}}+\sum_{a_{2}} \eta_{2} \delta_{b a_{2}} \delta_{c a_{2}}+\sum_{a_{3}} \eta_{3} \delta_{b a_{3}} \delta_{c a_{3}} \\
& +\eta_{4} \delta_{b a_{4}} \delta_{c a_{4}} .
\end{aligned}
$$

The RG flow Eqs. (C3) become 

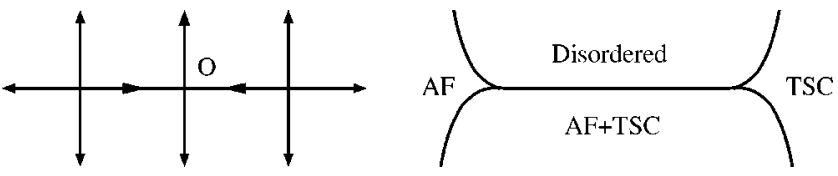

FIG. 18. RG flows in Eq. (51) for $N=4$. Point $\mathrm{O}$ has a symmetry $\mathrm{SO}(4) \times \mathrm{SO}(4)$.

$$
\begin{gathered}
\frac{d \eta_{1}}{d l}=\epsilon \eta_{1}-\frac{1}{2 \pi}\left(N-2+\frac{\eta_{1}^{2}-\eta_{2}^{2}-\eta_{3}^{2}}{\eta_{2} \eta_{3}}\right) \\
\frac{d \eta_{2}}{d l}=\epsilon \eta_{2}-\frac{1}{2 \pi}\left(N-2+\frac{\eta_{2}^{2}-\eta_{3}^{2}-\eta_{1}^{2}}{2 \eta_{1} \eta_{3}}-\frac{\eta_{4}}{2 \eta_{2}}\right) \\
\frac{d \eta_{3}}{d l}=\epsilon \eta_{3}-\frac{1}{2 \pi}\left(N-2+\frac{N-3}{2} \frac{\eta_{3}^{2}-\eta_{1}^{2}-\eta_{2}^{2}}{\eta_{1} \eta_{2}}-\frac{\eta_{4}}{2 \eta_{3}}\right) \\
\frac{d \eta_{4}}{d l}=\epsilon \eta_{4}-\frac{1}{2 \pi}\left(\frac{N-3}{2} \frac{\eta_{4}^{2}}{\eta_{2}^{2}}+\frac{\eta_{4}^{2}}{2 \eta_{3}^{2}}\right)
\end{gathered}
$$

The fixed $\mathrm{SO}(N) \times \mathrm{SO}(3)$ symmetric point of Eq. (C8) is described in the body of the text for $N \geqslant 5$. For $N=4$, the fixed point is stable with respect to arbitrary perturbations away from the $\mathrm{SO}(N) \times \mathrm{SO}(3)$ symmetric plane [incidentally, in this case the fixed point has a larger symmetry than expected, $\mathrm{SO}(4) \times \mathrm{SO}(4)]$. On the other hand, within the plane, it has one stable and one unstable direction. This suggests the RG flows and the phase diagram are shown schematically in Fig. 18.

Note that we have a whole line of direct transitions between the disordered and the TSC+AF phases. This whole line is controlled by the point $\mathrm{O}$ that has a high $\mathrm{SO}(4)$ $\times \mathrm{SO}(4)$ symmetry. This is quite remarkable: a higher symmetry appears not at a single point but at the whole transition line.

\section{APPENDIX D: NONUNITARY TRIPLET SUPERCONDUCTIVITY AND ANTIFERROMAGNETISM: LARGE $N$ ANALYSIS}

In Sec. VI A we pointed out that a free energy in Eq. (29) in the large $N$ limit should be considered with care, when $\tilde{u}_{1}+\tilde{u}_{2} / 3$ is close to zero. Here we assume that $\tilde{u}_{2}>0$, so, this requires negative $\tilde{u}_{1}$. The complications arise when the system goes outside the basin of attraction of the tetracritical fixed point, and the RG flows carry $\tilde{u}_{1}$ to large negative values. As we discuss below, this leads to a first order transition which is similar to what was suggested in Ref. 74 for the normal to $A_{1}$ transition in liquid ${ }^{3} \mathrm{He}$. We take

$$
\tilde{u}_{1}+\tilde{u}_{2} / 3=\frac{\delta}{4 N},
$$

where $\delta$ is positive and is of order $1 / N$. We now extend the calculations presented in Sec. VI A to the next order in $1 / N$. For all order parameters we separate expectation values and fluctuations

$$
\begin{gathered}
\boldsymbol{\Psi}=\left(\mathbf{a}_{T}+\imath \mathbf{b}_{T}, \sigma_{\psi}+a_{L}+\imath b_{1}, a_{0}+i \sigma_{\psi}+\imath b_{L}, a_{1}+\imath b_{0}\right) \\
\mathbf{N}=\left(\mathbf{N}_{T}, N_{0}, N_{1}, \sigma_{N}+N_{L}\right) .
\end{gathered}
$$

We can expand Eq. (29) to order $1 / N^{2}$ and obtain tadpole equations for $a_{L}$ and $N_{L}$. In addition to the counterterms and loops due to fluctuations of the transverse components, we need to include fluctuations of the longitudinal components. Note, that loops of longitudinal components may be terminated by bubble chains coming from $\widetilde{u}_{2}\left(\mathbf{a}_{T} \mathbf{b}_{T}\right) a_{0}$ vertices. We also need to include diagrams that arise from $\tilde{u}_{2}\left(\mathbf{a}_{T} \mathbf{b}_{T}\right) b_{1} a_{L}$ vertices. Special attention should be paid to diagonalization of propagators, since the free energy has terms which introduce mixing between fluctuating components in Eq. (D2).

If we want to absorb the cutoff dependence into renormalization of quadratic coefficient [compare to Eq. (38)], we need to define the latter relative to

$$
r_{c^{\prime}}=r_{c}-\left(40 \tilde{u}_{1}+24 \tilde{u}_{2}\right) \int_{0}^{\Lambda} \frac{d^{3} k}{(2 \pi)^{3}} \frac{1}{k^{2}}+\left(4 \tilde{u}_{1}+8 \tilde{u}_{2}\right) \frac{j}{2 \pi^{2}} \log \Lambda,
$$

where

$$
j=\frac{\tilde{u}_{2} N}{4} .
$$

Integrals in tadpole equations cannot be calculated exactly. Hence, we expand them in two cases: $j^{2} \gg 32 \widetilde{u}_{2} \sigma^{2}$ and $j^{2} \ll 32 \widetilde{u}_{2} \sigma^{2}\left[\sigma^{2}\right.$ corresponds to $\sigma_{N}^{2}$ or $\sigma_{\Psi}^{2}$ or $\left(\sigma_{N}^{2}+\sigma_{\Psi}^{2}\right) / 2 \mathrm{de}-$ pending on terms in the integrals]. Also, while solving final system of equations, expansions under conditions $\sigma_{\Psi}^{2} \gg \sigma_{N}^{2}$, $\sigma_{\Psi}^{2} \ll \sigma_{N}^{2}, \sigma_{\Psi}^{2} \approx \sigma_{N}^{2}$ were made. To be concrete, we took the values $\widetilde{u}_{1}=-1 /(4 N)+\delta /(4 N)$ and $\widetilde{u}_{2}=3 /(4 N)$.

Transition from disordered phase to superconductive and antiferromagnetic phases outside the vicinity of $r_{c^{\prime}}$ remains of the second order, though transition border shifts such that

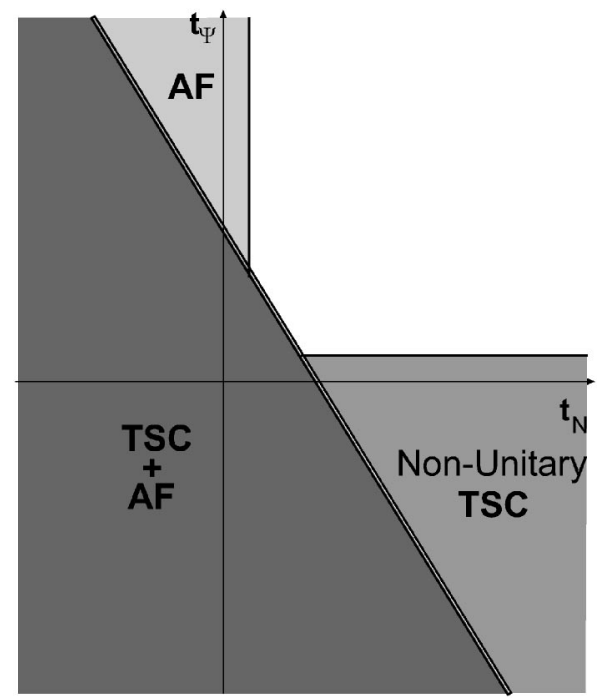

FIG. 19. Phase diagram for large $\mathrm{N}$ under conditions ((D1)). Solid line is a I order phase transition, dashed-II. 


$$
t_{N, \Psi}=\frac{15}{16 \pi^{2} N} \log \frac{16}{3} .
$$

In the vicinity of full $\mathrm{SO}(3) \times \mathrm{SO}(N)$ symmetry line $t_{N}$ $=t_{\Psi}$ we expanded equations under conditions

$$
\left|\sigma_{N}^{2}-\sigma_{\Psi}^{2}\right| \sim \sigma^{2} / N
$$

resulting in first order phase transition, limited by boundaries

$$
t_{N, L}+2 t_{\Psi, L}=0
$$

and

$$
t_{N, M}+2 t_{\Psi, M}=\frac{C_{0}^{2}}{4 N^{2} \delta},
$$

where $C_{0}=(1+3 \sqrt{3 / 2}) / \pi$.
Condition (D6) for solution obtained, appears to be valid not only for small deviations from the line of symmetry, but for entire line of transition. Thus solution [Eqs. (D7) and (D8)] is self-consistent in the entire region, resulting in phase diagram shown in Fig. 19. In comparison with solution of the first order expansion, boundary of mixed phase becomes a first order phase transition, and there is no angle between $N \Psi \leftrightarrow N$ and $N \Psi \leftrightarrow \Psi$ boundaries (which is $\propto \delta$ in first order expansion). Boundary of the basin of attraction of stable fixed point is determined by the validity of expansion for different conditions for $j^{2}$. In our case it is $N \delta \sim 1$.

On the other hand, expansion under condition $\sigma_{N}^{2} \gg \sigma_{\Psi}^{2}$ for $N \Psi \leftrightarrow N$ transition, and $\sigma_{N}^{2} \ll \sigma_{\Psi}^{2}$ for $N \Psi \leftrightarrow \Psi$ also results in self-consistent solution with phase transition boundary of a different geometry. On the boundary minor component drops to zero, while major almost does not change. In our opinion, this solution does not have a physical sense.
${ }^{1}$ T. Ishiguro, K. Yamaji, and G. Saito, Organic Superconductors (Springer, Berlin, 1998).

${ }^{2}$ D. Jérome, in Organic Conductors: Fundamentals and Applications, edited by J. P. Farges (Marcel Dekker, New York, 1994).

${ }^{3}$ L. Coleman, M. J. Cohen, D. J. Sandman, F. G. Yamagishi, A. Garito, and A. J. Haeger, Solid State Commun. 12, 1125 (1973).

${ }^{4}$ K. Bechgaard, C. S. Jacobsen, K. Mortensen, H. J. Pedersen, and N. Thorup, Solid State Commun. 33, 1119 (1980).

${ }^{5}$ A. Maaroufi, S. Flandrois, G. Fillion, and J. P. Morand, Mol. Cryst. Liq. Cryst. 119, 311 (1985).

${ }^{6} \mathrm{~S}$. Barišić and S. Brazovskii, in Recent Developments in Condensed Matter Physics, edited by J. T. Devreese (Plenum, New York, 1981).

${ }^{7}$ D. Jérome, A. Mazaud, M. Ribault, and K. Bechgaard, J. Phys. (Paris), Lett. 41, 95 (1980).

${ }^{8}$ K. Andres, F. Wudl, D. B. McWhan, G. A. Thomas, D. Nalewajek, and A. L. Stevens, Phys. Rev. Lett. 45, 1449 (1980).

${ }^{9}$ T. Takahashi, H. Kawamura, T. Teruo, Y. Maniwa, K. Murata, and G. Saito, J. Phys. Soc. Jpn. 58, 703 (1989).

${ }^{10}$ T. Vuletic, P. Auban-Senzier, C. Pasquier, S. Tomic, D. Jérome, M. Heritier, and K. Bechgaard, Eur. Phys. J. B 25, 319 (2002).

${ }^{11}$ A. Kornilov, V. M. Pudalov, Y. Kitaoka, K. Ishida, G. Q. Zheng, T. Mito, and J. S. Qualls, Phys. Rev. B 69, 224404 (2004).

${ }^{12}$ M. Dressel, Physica C 317, 89 (1999).

${ }^{13}$ M. Choi, P. Chaikin, and R. Greene, Phys. Rev. B 34, 7727 (1986).

${ }^{14}$ M. Choi, P. Chaikin, S. Huang, P. Haen, E. Engler, and R. Greene, Phys. Rev. B 25, 6208 (1982).

${ }^{15}$ S. Tomic, D. Jérome, D. Mailly, M. Ribault, and K. Bechgaard, J. Phys. Colloq. 44, C3 (1983).

${ }^{16}$ C. Coulon, P. Delhaes, J. Amiell, J. P. Manceau, J. Fabre, and L. Giral, J. Phys. (Paris) 43, 1721 (1982).

${ }^{17}$ A. A. Abrikosov, JETP Lett. 37, 503 (1983).

${ }^{18}$ I. Lee, M. J. Naughton, G. M. Danner, and P. M. Chaikin, Phys. Rev. Lett. 78, 3555 (1997)

${ }^{19}$ L. P. Gor'kov and D. Jérome, J. Phys. (Paris), Lett. 46, 646 (1985).

${ }^{20}$ I. Lee, D. Chow, W. Clark, M. Strouse, M. Naughton, P. Chaikin, and S. Brown, Phys. Rev. B 68, 092510 (2003).

${ }^{21}$ M. Takigawa, H. Yasuoka, and G. Saito, J. Phys. Soc. Jpn. 56, 873 (1987).

${ }^{22}$ H. I. Ha, J. I. Oh, J. Moser, and M. J. Naughton, Synth. Met. 137, 1215 (2003).

${ }^{23}$ N. Joo, P. Auban-Senzier, C. Pasquier, D. Jérome, and K. Bechgaard, Eur. Phys. J. B 40, 43 (2004).

${ }^{24}$ J. Oh and M. Naughton, Phys. Rev. Lett. 92, 067001 (2004).

${ }^{25}$ R. Brusetti, M. Ribault, D. Jérome, and K. Bechgaard, J. Phys. (Paris) 43, 801 (1982).

${ }^{26}$ D. Jaccard, H. Wilhelm, D. Jérome, J. Moser, C. Carcel, and J. M. Fabre, J. Phys.: Condens. Matter 13, L89 (2001).

${ }^{27}$ M. Maple, J. Magn. Magn. Mater. 177, 18 (1998).

${ }^{28}$ N. Mathur, F. Grosche, S. R. Julian, I. R. Walker, D. Freye, R. Haselwimmer, and G. G. Lonzarich, Nature (London) 394, 39 (1998).

${ }^{29}$ Y. Kitaoka, K. Ishida, Y. Kawasaki, O. Trovarelli, C. Greibel, and F. Steglich, J. Phys.: Condens. Matter 13, L79 (2001).

${ }^{30}$ S. Zhang, Science 275, 1089 (1997).

${ }^{31}$ S. Meixner, W. Hanke, E. Demler, and S.-C. Zhang, Phys. Rev. Lett. 79, 4902 (1997).

${ }^{32}$ R. Eder, A. Dorneich, M. Zacher, W. Hanke, and S.-C. Zhang, Phys. Rev. B 59, 561 (1999).

${ }^{33}$ S. Murakami and N. Nagaosa, J. Phys. Soc. Jpn. 69, 2395 (2000).

${ }^{34}$ D. J. Scalapino, S.-C. Zhang, and W. Hanke, Phys. Rev. B 58, 443 (1998).

${ }^{35}$ H.-H. Lin, L. Balents, and M. Fisher, Phys. Rev. B 58, 1794 (1998).

${ }^{36}$ R. S. Markiewicz and M. T. Vaughn, Phys. Rev. B 57, R14052 (1998).

${ }^{37}$ C. Yang, Phys. Rev. Lett. 63, 2144 (1989).

${ }^{38}$ S. Zhang, Phys. Rev. Lett. 65, 120 (1990).

${ }^{39}$ P. A. Lee, N. Nagaosa, T.-K. Ng, and X.-G. Wen, Phys. Rev. B 57, 6003 (1998).

${ }^{40}$ C. Nayak, Phys. Rev. B 62, 4880 (2000).

${ }^{41}$ S. Murakami, N. Nagaosa, and M. Sigrist, Phys. Rev. Lett. 82, 2939 (1999).

${ }^{42}$ C. Henley, Phys. Rev. Lett. 80, 3590 (1998). 
${ }^{43}$ S. Rabello, H. Kohno, E. Demler, and S. Zhang, Phys. Rev. Lett. 80, 3586 (1998).

${ }^{44}$ A. P. Mackenzie and Y. Maeno, Rev. Mod. Phys. 75, 657 (2003).

${ }^{45}$ M. Braden, P. Steffens, Y. Sidis, J. Kulda, P. Bourges, S. Hayden, N. Kikugawa, and Y. Maeno, Phys. Rev. Lett. 92, 097402 (2004).

${ }^{46}$ O. Friedt, P. Steffens, M. Braden, Y. Sidis, S. Nakatsuji, and Y. Maeno, Phys. Rev. Lett. 93, 147404 (2003).

${ }^{47} \mathrm{C}$. Bourbonnais and D. Jérome, in Proceedings of International Conference of Science and Technology of Synthetic Metals (ICSM'98) (Elsevier, New York, 1999).

${ }^{48}$ M. Dressel, K. Petukhov, B. Salameh, P. Zornoza, and T. Giamarchi (2004), preprint cond-mat/0409322.

${ }^{49}$ J. Sólyom, Adv. Phys. 28, 201 (1979).

${ }^{50}$ T. Giamarchi and H. Schulz, Phys. Rev. B 39, 4620 (1989).

${ }^{51}$ A. Rozhkov and A. Millis, Phys. Rev. B 66, 134509 (2002).

${ }^{52}$ S. Carr and A. Tsvelik, Phys. Rev. B 65, 195121 (2002).

${ }^{53}$ T. Giamarchi, Physica B 230-232, 975 (1997).

${ }^{54}$ N. Thorup, G. Rindolf, H. Soling, and K. Bechgaard, Acta Crystallogr., Sect. B: Struct. Crystallogr. Cryst. Chem. 37, 1236 (1981).

${ }^{55}$ D. Podolsky, E. Altman, T. Rostunov, and E. Demler, cond-mat/ 0403406, Phys. Rev. Lett. (to be published).

${ }^{56}$ J. B. Torrance, H. J. Pedersen, and K. Bechgaard, Phys. Rev. Lett. 49, 881 (1982).

${ }^{57}$ K. Mortensen, Y. Tomkiewicz, and K. Bechgaard, Phys. Rev. B 25, 3319 (1982).

${ }^{58}$ C. Bourbonnais, P. Stein, D. Jérome, and A. Moradpour, Phys. Rev. B 33, 7608 (1986).

${ }^{59}$ K. Efetov, Sov. Phys. JETP 54, 583 (1981).

${ }^{60}$ L. Degiorgi, V. Vescoli, W. Henderson, G. Grüner, and L. K. Montgomery, J. Phys. IV (Paris) 10, 3 (2000).

${ }^{61}$ A. Schwartz, M. Dressel, G. Grüner, V. Vescoli, L. Degiorgi, and T. Giamarchi, Phys. Rev. B 58, 1261 (1998).

${ }^{62}$ Y. Zhang, E. Demler, and S. Sachdev, Phys. Rev. B 66, 094501 (2002).

${ }^{63}$ This is in contrast with rational fillings, such as $\nu=1 / 4$, for which translational symmetry allows terms of the form

$$
\operatorname{Re}\left[e^{i \theta}(\boldsymbol{\Phi})^{4 n}\right]
$$

for any positive integer $n$ and for an arbitrary fixed phase $\theta$. These terms tend to pin the phase of the SDW order, and they reduce the $\mathrm{SO}(2)$ symmetry of translations to the discrete group $Z_{4}$. Note that $\mathrm{SO}(4)_{\text {isospin }}$ symmetry does not allow pinning terms such as these. This is because the operator $\Lambda_{z}=Q_{+}$ $-Q_{-}$generates continuous rotations between the real and imaginary parts of $\boldsymbol{\Phi}$, Eq. (12).

${ }^{64}$ A. Leggett, Rev. Mod. Phys. 47, 331 (1975).

${ }^{65}$ C. Yang and S. Zhang, Mod. Phys. Lett. B 4, 759 (1990).

${ }^{66}$ Triplet pairing in Bechgaard salts does not have orbital degree of freedom. Quasi-one-dimensional electron band structure requires $\boldsymbol{\Psi}(\mathbf{p}) \propto p_{x}$, where $x$ is the direction parallel to the chains. This provides considerable simplification in writing effective models, such as the Ginzburg-Landau free energy ((30)).

${ }^{67}$ P. Anderson, Basic Notions of Condensed Matter Physics (Benjamin Cummings, Reading, 1984).

${ }^{68}$ M. Fisher and D. Nelson, Phys. Rev. Lett. 32, 1350 (1974).

${ }^{69}$ J. Kosterlitz, D. Nelson, and M. Fisher, Phys. Rev. B 13, 412 (1976).
${ }^{70}$ E. Demler, W. Hanke, and S. Zhang (2004), preprint cond-mat/ 0405038.

${ }^{71}$ P. Chaikin and T. C. Lubensky, Principles of Condensed Matter Physics (Cambridge University Press, London, 2000).

${ }^{72}$ J. Zinn-Justin, Quantum Field Theory and Critical Phenomena (Oxford University Press, Oxford, 1993).

${ }^{73}$ S. K. Ma, Modern Theory of Critical Phenomena (Benjamin Cummings, Reading, 1976).

${ }^{74}$ D. Bailin, A. Love, and M. Moore, J. Phys. C 10, 1159 (1977).

${ }^{75}$ D. Friedan, Ann. Phys. (N.Y.) 163, 318 (1985).

${ }^{76}$ P. Azaria, B. Delamotte, and T. Jolicoeur, Phys. Rev. Lett. 64, 3175 (1990).

${ }^{77}$ H. Kawamura, J. Phys. Soc. Jpn. 60, 1839 (1991).

${ }^{78}$ F. David and T. Jolicoeur, Phys. Rev. Lett. 76, 3148 (1996).

${ }^{79}$ H. Kawamura, J. Phys.: Condens. Matter 10, 4707 (1998).

${ }^{80}$ H. Diep and D. Loison, J. Appl. Phys. 76, 6350 (1994).

${ }^{81}$ B. Delamotte, D. Mouhanna, and M. Tissier, Phys. Rev. B 69, 134413 (2004).

${ }^{82}$ H. Kawamura, J. Phys. Soc. Jpn. 61, 1299 (1992).

${ }^{83}$ H. Kunz and G. Zumbach, J. Phys. A 26, 3121 (1993).

${ }^{84}$ H. Kawamura, J. Phys. Soc. Jpn. 54, 3220 (1995).

${ }^{85}$ A. Mailhot, M. L. Plumer, and A. Caillé, Phys. Rev. B 50, 6854 (1994).

${ }^{86}$ T. Bhattacharya, A. Billoire, R. Lacaze, and T. Jolicoeur, J. Phys. I 4, 181 (1994).

${ }^{87}$ D. Loison and H. T. Diep, Phys. Rev. B 50, 16453 (1994).

${ }^{88}$ A. Peles and B. W. Southern, Phys. Rev. B 67, 184407 (2003).

${ }^{89}$ D. Loison and K. D. Schotte, Eur. Phys. J. B 14, 125 (2000).

${ }^{90}$ D. Loison, Physica A 275, 207 (2000).

${ }^{91}$ M. Itakura, J. Phys. Soc. Jpn. 72, 74 (2003).

${ }^{92}$ M. Tissier, B. Delamotte, and D. Mouhanna, Phys. Rev. Lett. 84, 5208 (2000).

${ }^{93}$ S. Sachdev, Quantum Phase Transitions (Cambridge University Press, London, 1999).

${ }^{94}$ E. Demler, S. Zhang, N. Bulut, and D. Scalapino, Int. J. Mod. Phys. B 10, 2137 (1996).

${ }^{95}$ S. Zhang, J. Hu, E. Arrigoni, W. Hanke, and A. Auerbach (1999), preprint cond-mat/9904142.

${ }^{96}$ R. S. Markiewicz, C. Kusko, and M. T. Vaughn, in High Temperature Superconductivity, edited by S. E. Barnes, J. Ashkenazi, J. L. Cohn, and F. Zuo (AIP, Woodbury, 1999).

${ }^{97}$ E. Altman and A. Auerbach, Phys. Rev. B 65, 104508 (2002).

${ }^{98}$ S. Sachdev, Rev. Mod. Phys. 75, 913 (2003).

${ }^{99}$ J. F. Kwak, Mol. Cryst. Liq. Cryst. 79, 111 (1982).

${ }^{100}$ H. Bando, K. Oshima, M. Suzuki, H. Kobayashi, and G. Saito, J. Phys. Soc. Jpn. 51, 2711 (1982).

${ }^{101}$ B. Brusetti, P. Garoche, D. Jérome, and K. Bechgaard, J. Phys. (France) Lett. 43, 147 (1982).

${ }^{102}$ L. P. Gor'kov and A. G. Lebed', 0J. Phys. (Paris), Lett. 45, 433 (1984).

${ }^{103}$ N. Dupuis, G. Montambaux, and C. A. R. S. de Melo, Phys. Rev. Lett. 70, 2613 (1993).

${ }^{104}$ V. M. Yakovenko and H.-S. Goan, J. Phys. I 6, 1917 (1996).

${ }^{105}$ V. Vescoli, L. Degiorgi, W. Henderson, G. Gruner, K. Starkey, and L. Montgomery, Science 281, 1181 (1998).

${ }^{106}$ C. Jacobsen, D. B. Tanner, and K. Bechgaard, Phys. Rev. B 28, 7019 (1983).

${ }^{107}$ P. Grant, J. Phys. (Paris) 44, C3 (1983).

${ }^{108}$ E. Demler, H. Kohno, and S. Zhang, Phys. Rev. B 58, 5719 (1998). 
${ }^{109}$ E. W. Carlson, V. J. Emery, S. A. Kivelson, and D. Orgad, in The Physics of Conventional and Unconventional Superconductors, edited by K. Bennemann and J. Ketterson (Springer, Berlin, 2002).

${ }^{110}$ D. P. Arovas, A. J. Berlinsky, C. Kallin, and S.-C. Zhang, Phys. Rev. Lett. 79, 2871 (1997).

${ }^{111}$ E. Demler, S. Sachdev, and Y. Zhang, Phys. Rev. Lett. 87, 067202 (2001).

${ }^{112}$ H. Kang, P. Dai, J. Lynn, M. Matsuura, J. Thompson, S. Zhang, D. Argyriou, Y. Onose, and Y. Tokura, Nature (London) 423, 522 (2003).

${ }^{113}$ P. Garoche, R. Brusetti, D. Jerome, and K. Bechgaard, J. Phys. (Paris), Lett. 43, 147 (1982).

${ }^{114}$ S. Katano, M. Sato, K. Yamada, T. Suzuki, and T. Fukase, Phys. Rev. B 62, R14677 (2000).

${ }^{115}$ B. Lake et al., Science 291, 1759 (2001).
${ }^{116}$ B. Lake et al., Nature (London) 415, 299 (2002).

${ }^{117}$ B. Khaykovich, Y. Lee, R. Erwin, S.-H. Lee, S. Wakimoto, K. Thomas, M. Kastner, and R. Birgeneau, Phys. Rev. B 66, 014528 (2002).

${ }^{118}$ N. Curro, C. Milling, J. Haase, and C. Slichter, Phys. Rev. B 62, 3473 (2000).

${ }^{119}$ V. Mitrović, E. E. Sigmund, W. P. Halperin, A. P. Reyes, P. Kuhns, and W. G. Moulton, Phys. Rev. B 67, 220503 (2003).

${ }^{120}$ K. Kakuyanagi, K. Kumagai, and Y. Matsuda, J. Phys. Chem. Solids 63, 2305 (2002).

${ }^{121}$ R. I. Miller et al., Phys. Rev. Lett. 88, 137002 (2002).

${ }^{122}$ Y. Bazaliy, E. Demler, and S. Zhang, Phys. Rev. Lett. 79, 1921 (1997).

${ }^{123}$ D. Scalapino, Phys. Rev. Lett. 24, 1052 (1970).

${ }^{124}$ C. Bourbonnais, F. Creuzet, D. Jérome, K. Bechgaard, and A. Moradpour, J. Phys. (Paris), Lett. 45, 755 (1984). 